Canadian Journal of Fisheries and Aquatic Sciences

Canadian

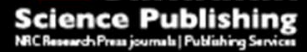

Journal canadien des sciences halieutiques et aquatiques

\title{
Improving stock assessments through data prioritization
}

\begin{tabular}{|r|l|}
\hline Journal: & Canadian Journal of Fisheries and Aquatic Sciences \\
\hline Manuscript ID & cjfas-2015-0398.R1 \\
\hline Manuscript Type: & Article \\
\hline Date Submitted by the Author: & 03-Jan-2016 \\
\hline Complete List of Authors: & $\begin{array}{l}\text { Siegfried, Kate; National Marine Fisheries Service - NOAA } \\
\text { Williams, Erik; National Marine Fisheries Service - NOAA } \\
\text { Shertzer, Kyle; National Marine Fisheries Service - NOAA } \\
\text { Coggins, Lewis; Yukon Delta National Wildlife Refuge, }\end{array}$ \\
\hline Keyword: & $\begin{array}{l}\text { STOCK ASSESSMENT < General, Data Prioritization, Sampling strategy } \\
\text { evaluation, simulation study }\end{array}$ \\
\hline \multicolumn{2}{|c}{} \\
\hline
\end{tabular}

SCHOLARONE ${ }^{m}$

Manuscripts 
$5{ }^{1}$ NOAA/National Marine Fisheries Service, 101 Pivers Island Rd, Beaufort, NC, 28516., USA (252)728-8721, Fax: (252) 728-8784, E- mail:Kate.Siegfried@noaa.gov,

7 Erik.Williams@noaa.gov, Kyle.Shertzer@noaa.gov, ${ }^{2}$ U.S. Fish \& Wildlife Service, Bethel, AK, USA. E-mail: lewis_coggins@fws.gov *Corresponding author

17 Keywords: stock assessment, sampling strategy evaluation, data prioritization, simulation study 
19 Abstract: The need for "better data" is a common response of stakeholders and managers when 20 confronted with the uncertainty of advice resulting from quantitative stock assessments. Most

21 contemporary stock assessments are based on an integrated analysis of multiple data types each

22 with their associated cost to collect. Data collection resources are inevitably limited, therefore it

23 is important to quantify the relative value of increased sampling for alternative data types in

24 terms of improving stock assessments. We approached this universal problem using a

25 simulation study of a hypothetical, amalgam species developed from eight separate stock

26 assessments conducted in the southeastern U.S. Atlantic waters. We simulated a population and a

27 stock assessment from the amalgam species, and then individually improved alternative data

28 types (indices, age compositions, landings and discards), by either increasing precision or sample

29 size. We also simulated the effects of increased sampling for alternative groupings of data that might be collected in concert (e.g., commercial, all recreational, or survey). Our results show

31 that, for the snapper-grouper complex we modeled, age composition data have the largest effect

32 on the accuracy of assessments, with commercial age compositions being the most influential.

33 This is due in part to the relative paucity of age composition data for many southeast U.S. marine

34 stocks, so that modest increases in collection efforts have relatively high benefits for age-based 35 assessment models currently in use for the region. Though this study used data from a particular

36 region of the U.S., our investigative framework is broadly applicable for quantitatively

37 evaluating the benefits of improved data collection in terms of the precision of stock assessments 38 in any region. 


\section{Introduction}

42 Fisheries management often depends on stock assessments to inform decisions about potential

43 catch limits relative to the dynamics of the stock. Uncertainty, or a lack of data, may necessitate

44 a more precautionary approach to management, which often leads to lower available yields.

45 Thus, more certainty about the data included in a stock assessment is highly desirable. When the

46 data are available, stock assessments are an integrated analysis of those multiple data sources,

47 including landings and discards, life history information, size and age compositions, and indices

48 of abundance, each of which has an associated level of uncertainty (Maunder and Punt 2013).

49 For example, fishery catches and discards may be imprecise due to low observer coverage or

50 sporadic port sampling. Life history data used to inform natural mortality, growth, and

51 reproductive output may be sparse or borrowed from other species (Punt et al. 2011). Age

52 composition data require considerable post-collection processing that varies greatly across

53 laboratories. Fishery-independent indices require extensive field survey efforts and consistent

54 funding to maintain the integrity of the relative abundance time series. Research

55 recommendations compiled during assessments inevitably contain requests for more of each of

56 these data types, yet few efforts have focused on how best to prioritize data collection efforts.

57 Even in the face of an increasing demand for assessments, only minimal improvements to data

58 collection may be economically feasible. Thus, there is a pressing need to develop approaches

59 for prioritizing data in terms of its utility for stock assessments (Magnusson and Hilborn 2007).

When faced with the question of which data type to collect more of, no clear priority

61 exists. Scientists want the assessment to reflect the population and characterize uncertainty as

62 well as possible, but there have only been a few studies that address the effects of various data 
sources on assessment results (Powers and Restrepo 1993; Yin and Sampson 2004; Szuwalski and Punt 2012).

Data collection efforts, as well as the perceived needs for additional data types, vary considerably across geographic regions. In regions where catches are hardly characterized (e.g. the Caribbean), data collection priorities are clearer. However, regions that have conducted multiple age-structured stock assessments have various data deficits that need to be filled. As an example, fishery scientists working on species in the Atlantic waters of the southeastern U.S. repeatedly encounter a lack of fishery-independent survey data, discards are difficult to characterize, and annual age composition data are insufficient. In addition, a high proportion of the landings and discards are taken by the recreational fleet, which is inherently difficult to monitor. Nevertheless, age-structured assessments are regularly used to inform fishery management decisions. The goal of this work is to develop a framework for providing objective guidance about prioritizing data sources for fish stock assessments. Using simulation, we evaluate which data types are most effective at improving assessment accuracy by modeling the effect of improving each data source. Although our study design is applied to data from a single region, the approach is general and could be applied in other regions.

\section{Methods}

We simulated a hypothetical population (i.e., an amalgam species) based on information available for actual stocks from the snapper-grouper complex that have been individually assessed in the Atlantic waters off the southeastern U.S. We reviewed the assessed stocks in the region and averaged the results of eight stocks: Vermilion Snapper (Rhomboplites aurorubens), Red Porgy (Pagrus pagrus), Black Sea Bass (Centropristis striata), Red Snapper (Lutjanus 
campechanus), Greater Amberjack (Seriola dumerili), Red Grouper (Epinephelus morio), Snowy Grouper (Hyporthodus niveatus), and Gag (Mycteroperca microlepis) (SEDAR 2008, 2009, 2010, 2012a, b, 2013a, b, 2014). The combined results from the eight assessments provide an amalgam stock that represents the snapper/grouper complex from 1963 to 2012 (Table 1).

We modeled ages 1 to 16 years using annual time steps, with an average maximum age of 16. We used an averaged von Bertalanffy (von Bertalanffy 1938) growth curve $\left(L_{\infty}=792 \mathrm{~mm}, k\right.$ $=0.18, t_{0}=-1.36$ ) (Figure 1), and an average age of $50 \%$ maturity of 2.25 years. For the growth curve, we excluded Greater Amberjack, as its growth pattern seemed to diverge from the other species. However, its other life history parameters were similar to the other species in the complex. We modeled two fleets (recreational and commercial) and two relative abundance indices (one fishery-dependent and one fishery-independent) each based on the averaged trends of the eight stocks.

According to the amalgam species data, the recreational index data were typically available beginning in 1981 and ran continuously through the terminal year. The fisheryindependent index began in 1996 and also ran through the terminal year of the model (Figure 2). The average commercial fishery landings were assumed to be known very well, with a coefficient of variation $(\mathrm{CV})$ of 0.05 . The $\mathrm{CV}$ for the recreational fishery was related to the percent standard error (PSE) provided by a Marine Recreational Information Program (MRIP) catch data query (Personal communication from the National Marine Fisheries Service, Fisheries Statistics Division. [Queried November 2014]). Discards for each fishery were available annually since 1992. Age composition data were compiled from the survey (9 years), commercial fleet (12 years), and the recreational fleet (12 years). We did not simulate length 
107 108 109 110

111

112

113

114

115 116

117 118 119 120

composition data as it has been shown to provide relatively little information to estimate population dynamics for snapper-grouper species in the region (SEDAR 2012b).

We use a catch-age model called the Beaufort Assessment Model (BAM) that is commonly used for assessments in the southeastern U.S. as the operating model for the simulations (Williams and Shertzer 2015). Briefly, BAM is a statistical catch-age model implemented with the AD Model Builder software (Fournier et al. 2012), and offers the flexibility of a customizable source code while providing the basic constructs and functions of most other age-based assessment models (e.g., Beverton-Holt stock recruitment relationship, a variety of age-based selectivity options, weighting options for the likelihood components, penalties to constrain parameter spaces, etc.). We only altered the operating model between runs to change either the precision or the sample size of each data type (Table 2).

We used the average of the parameters from each of the stock assessments (see Table 2) to create a set of input parameters to simulate a "true" population with the operating model. Each true population data set was then fit with the same operating model using optimization.

Our algorithm for the simulation was as follows:

Step 1: Configure the operating model with fixed parameters to create a simulated data set using BAM by imputing the mean of each of the following parameters from our amalgam stock: start year, maximum age, years of recruitment deviations, catch per unit effort time series (CPUEs), landings time series, von Bertalanffy growth parameters, steepness, selectivities, catchabilities, weight at age, maturity at age, and fishing mortality trends (Figures 3-4, see the Supplementary Material for the data input file). We used 
2012 as the terminal year, which was the last assessment year in our amalgam stock. The mean selectivity for each sector was used for the amalgam species: commercial, recreational, survey, and discards (Figure 5). We generated age compositions with a multinomial distribution using the mean sample size and the number of years that composition data were available for our amalgam species. We then allow for random recruitment assuming lognormal deviations (Fogarty et al. 1991).

Step 2: Add observation error to mimic actual assessments. Observation error was added to the recreational CPUE and survey indices based on the average CV of these two data types across the eight prior assessments. Error was also added to the composition data by increasing or decreasing the sample sizes used in the multinomial distribution.

Step 3: Create the operating data set using all stochastic components from step 2 and then use BAM to estimate model parameters of the stochastic, simulated data set.

Step 4: Collect the results from the estimation in step 3, and repeat for the desired number $(\mathrm{N}=2000)$ of bootstrap iterations.

We tracked model estimates of virgin recruitment, steepness, Acceptable Biological Catch (ABC), and status indicators (spawning stock biomass sufficient to produce maximum sustainable yield (MSY, $\mathrm{SSB}_{\mathrm{MSY}}$ ), fishing mortality required to harvest MSY ( $\mathrm{F}_{\mathrm{MSY}}$ ), and minimum stock size threshold (MSST)). ABC was calculated for a single year following the terminal year of the assessment using terminal biomass and estimates of fishing mortality from 
148 149 150 151 153 154 155 156 157 158 159 160 161 162

each fishery. MSST is used as an indicator by the South Atlantic Fishery Management Council of an overfished stock and was calculated as $0.5 * \mathrm{SSB}_{\mathrm{MSY}}$.

We focused on data types considered to be the best candidates for improved collection: landings, discards, age compositions, and fishery independent data (i.e., CPUE and age composition). The relative cost of each type of data is known, and programs are currently in place that could improve their quality. For example, a larger budget for the fishery-independent survey would likely translate into more sea days and more samples taken, which would translate directly into the type of precision and sample size improvements modeled here. After initial simulations improving each individual data type separately (i.e., commercial discards, commercial age compositions, recreational discards, recreational landings, recreational age compositions, recreational CPUE, survey precision, survey age compositions), we conducted additional simulations where data types were grouped as 'commercial', 'recreational', and 'survey.' In each of the groupings, the CVs of landings, discards, and indices were decreased by four-fold and the sample sizes of age compositions were increased by five-fold. The values fourfold and five-fold were somewhat arbitrary but were chosen based on the extremes of what is considered practical for data collection efforts in the southeastern U.S. For example, because the amount of age data available in prior assessments was relatively small, we deemed it feasible to collect five times more otoliths above current efforts. But given current efforts to monitor landings and discards in our region, improving the precision of these data types by more than four-fold was considered impractical. The separate date types were grouped as: recreational landings and all discards, all age compositions, all survey data, all commercial data (except landings), all commercial data, and all indices (see Table 2 for the particulars of each scenario). 
For each scenario, we generated $\mathrm{N}=2000$ bootstrap replicates, which was sufficient for

171 the means of all estimated parameters to converge to stable values. However, one parameter,

172 steepness, proved difficult to estimate in $\sim 7-12 \%$ of replicates, and was estimated at its upper

173 bound of 0.99, a situation common in stock assessment (Conn et al. 2010). These runs were

174 culled before summarizing the results, because in practice steepness hitting an upper bound

175 would not be considered a valid run for assessment purposes. However, we report the proportion

176 of runs culled for each scenario (Table 3), as we consider it one indicator regarding the value of

177 improved data.

Steps two through four were repeated as each data type was improved either by

179 decreasing the CV or increasing the age composition sample sizes. Parameter estimates and 180 management quantities of each assessment fitted to improved data were compared to those of

181 the initial base model estimates using the mean relative error across all bootstrap replicates, $R E_{i}$

$182=\left(\hat{a}_{i} / a_{i}\right)-1$, where $\hat{a}_{i}$ is the estimated value of parameter $i$ and $a_{i}$ is the true value of parameter $i$.

184 Results

The base model run (i.e., without data improvements; see row 1 of Table 3 )

186 overestimated the biomass benchmark $(R E=0.38)$, and underestimated the fishing status

187 benchmark $(R E=-0.67)$. The estimates of the reference points show a positive bias for the base

188 case. The bias is the result of the variation put into the data and the sample sizes used. If the

189 variation is reduced and sample size increased prior to the data improvements, the model fits the

190 inputs exactly. Steepness was well-estimated in the base case as was the $\mathrm{ABC}(R E=-0.01$ and 
1910.05 , respectively). Time series of predicted landings and discards by fishery replicated the 192 general patterns observed across the eight assessed species (Fig. 6). Simulations with

193 improvements to the individual data types reduced the $R E$ of model estimates by $10-49 \%$ (Table

194 3). Improvements (i.e., increased effective sample sizes) in both the recreational and the 195 commercial age compositions had the greatest effect on model accuracy (23-68\%). For example, improved commercial age compositions reduced the estimation error of the biomass benchmarks and $R_{0}$ by a considerable amount (12.5 - 47\%), while improved recreational age compositions had the most effect on the estimates of $R_{0}$, MSY, and ABC (12.5 - 60\%).

Overall the grouped components reduced the $R E$ of model estimates by $22-70 \%$.

200

201

202

203

204

205

206

207

208

209

210

211

212

Specifically, the grouped composition data improvements had the largest effect on model accuracy overall, which is consistent with the results from the improvement of individual components. The effects were approximately double that of the individual component runs.

However, the survey components grouping showed an effect that was nearly absent in the runs of their individual components. The improvement of all survey components had a large effect on both the biomass and fishing benchmark estimates (RE decreased by $37 \%$ and $70 \%$ respectively), surpassing the effect of the all-composition data grouping for those two reference points. In general, probability density functions (pdfs) of key management quantities derived from the assessment illustrate how improved data collection can influence fishery management advice (Fig. 7, Supplementary Material). For example, when all age composition data were improved, the relative error in the fishing benchmark $\left(\mathrm{F} / \mathrm{F}_{\mathrm{msy}}\right)$ and the biomass benchmark $\left(\mathrm{SSB} / \mathrm{SSB}_{\mathrm{msy}}\right)$ decreased by $58 \%$ and $37 \%$, respectively, while the relative error in the estimated $\mathrm{ABC}$ was near zero. For the fishing benchmark in particular, improvements in the age composition data altered 
213 the qualitative determination of stock status from overfishing to not overfishing (Fig. 7). For the

214 amalgam species considered here, the estimated ABC was about 10\% larger for models

215 simulating improvements in the age composition data. Further improvements in the age

216 composition data decreased the presence of outliers and resulted in a narrower distribution about

217 the median (i.e. greater precision). The pdfs for all scenarios can be found in the Supplementary

218 Material.

219 Discussion

Our study provides a framework to generate specific and objective guidance to fishery

221 scientists and managers who must decide how to allocate limited monitoring and research

222 resources towards improving stock assessments. Improving accuracy would provide several

223 benefits, including better characterizing the stock dynamics and reducing the uncertainty in

224 management regulations. In U.S. fisheries management, reduced uncertainty in the assessment

225 generally results in larger catch limits, providing obvious manager and stakeholder benefits.

Our results are interpreted within the framework of the way assessments are conducted

227 and the current data availability in the southeastern U.S. For example, the data could have been

228 improved further (e.g., index CVs down to 5\% or samples sizes in the 1000 s per year as is the

229 case in other regions), however, it would be an impractical result. It would not be possible to

230 expand the current otolith sampling, survey or observer programs within a reasonable timeframe

231 in order to achieve those improvements. Therefore, we used our judgement, based on the

232 evidence provided in over a decade of snapper/grouper assessments, to improve the data by

233 realistic increments for the region. The feasibility of data improvement will differ across regions

234 depending on the current data collection programs. 
Assessments conducted in the southeastern U.S. are dominated by fishery-dependent

data. Commercial landings data are treated as known with high precision because there are often both trip ticket and logbook programs in place. There is also a need to anchor the model at the reported landings values, though the historical reconstruction of landings time series are inherently less certain. Commercial discards are much less certain and often estimated using logbook or observer data where available. General recreational landings are assumed less precise, and are estimated using an angler intercept survey. The angler intercepts are variable 242 and bring significant uncertainty to the model. Recreational effort is estimated using a phone survey (Andrews et al. 2014), which has historically suffered from a low response rate. This has motivated the Marine Recreational Information Program (MRIP) to switch to a mail survey to 245 decrease uncertainty around the landings and effort estimates. However, our study showed that 246 the most influential portion of recreational data for assessment purposes in the region is the 247 composition data. The improvement of the recreational discards also had an effect on the fishing 248 benchmark estimate. Both recreational and commercial discards are estimated with very large 249 CVs and are rarely verified with observer data. For our simulation, we used the same selectivity 250 for the recreational and commercial fisheries discards because of a lack of information to 251 differentiate the two. Due to the fact that much of the recreational fishing for most of these 252 species is done using similar gear and in similar depths, there are few possibilities for 253 recreational fisherman to discard different age classes. However, we did not model only 254 undersized fish as being discarded. The dome-shaped selectivity we used takes into account both 255 the closures and undersized fish. The difficulty specifying discard selectivity may have a very 256 small impact on the assessment, as our study suggests that improving the commercial discard 257 data had almost no effect on the model accuracy. 
Fish stocks in the Atlantic waters of the southeastern US have not been adequately

259 sampled by a fishery independent survey in the past (e.g. Rindone et al. 2015). When such data

260

261

262

263

264

265

266

267

268

269

270

271

272

273

274

275

276

277

278

279

280

are used in assessments, corresponding age compositions are typically available but noisy. In practice, we use length compositions only when age compositions are absent because they are often too noisy to provide informative demographic information to the model. Therefore we did not attempt to model length compositions in this study. None of the survey components has a noticeable effect individually, but the survey grouping was the second most influential data source in our study. While the composition grouping improved the biomass benchmarks, virgin recruitment, and MSY estimates, the survey grouping improved the fishing benchmark. It should be noted that the component grouping also had an effect on the fishing benchmark, but the relative error improvement was slightly less (58\% vs. $70 \%)$.

A few other studies have investigated the prioritization of data collection or improvements in different ways. Powers and Restrepo (1993) investigated how increasing precision in some of the data inputs changed the outcome of the assessment model used for King Mackerel (Scomberomorus cavalla) management in the Gulf of Mexico. Their study did not contain a simulation component, so it cannot be known whether the changes in the outcomes were any closer to the truth. If the true population dynamics are not known, results cannot be measured in terms of accuracy, but rather the desirability of the assessment outcome. Szuwalski and Punt (2012) used a simulation framework to determine research priorities in light of multiple model uncertainties. Using Eastern Bering Sea Snow Crab (Chionoecetes opilio), they increased the precision on the survey CPUE and the number of samples to develop the growth curve. This improved the estimability of some parameters; however their results are much more applicable to a length-structured model. The current study is most similar to Yin and Sampson's (2004), with 
281 a few key differences. Their study used a fractional factorial design to evaluate how input data

282

283

284

285

286

287

288

289

290

291

292

293

294

295

296

297

298

299

300

301

302

errors and the characteristics of the stock (with a focus on Pacific coast groundfish using the

Stock Synthesis program (Methot 2000)) affected the bias and precision of the model outcomes.

We used the BAM model, and formed an amalgam species data set to represent species in the Atlantic waters of the southeastern U.S. We used sample sizes and CVs that are reasonable for our region, which are lower than Yin and Sampson's (2004) composition sample size and a larger range of CVs than theirs for the survey CPUE. Nonetheless, we have one key result the same: age composition sample size is very influential on the accuracy of assessment outcomes.

For our region, we demonstrated that the individual components as well as the landings and discards grouping had very little impact on model accuracy. The composition grouping had the biggest effect on the accuracy of the assessment, followed by all survey components. Of the individual component runs, the commercial composition data was most influential followed by the recreational compositions. Interpreting the results in light of the cost of improving the data is encouraging. Composition data are relatively inexpensive when an aging lab is already established and operational. All otoliths have a similar cost to process but not necessarily to collect. A recreational otolith may be more expensive to collect because the process by which a port sampler gathers otoliths is typically opportunistic. Consequently, rather than additions to the daily sampling, data improvement may require additional days of sampling. Commercial composition data are easier to gather through standard sampling procedures at the dealers or fish houses. Compared to the cost of increasing a recreational effort sampling program, increasing fishery observer coverage, or increasing sampling days of a fishery-independent survey, composition data require the smallest investment with the biggest return. 
Our results come with a variety of caveats. Most importantly, the results are dependent

304

305

306

307

308

309

310

311

312

313

314

315

316

317

318

319

320

321

on the combination of data sources used for the simulation. In other words, the history of

removals, the biology of the stock and the availability of the different data sources through time will be unique by region and stock and will likely result in different data prioritization results.

As an example, our amalgam stock had a very small proportion of the biomass removed through discarding. Other stocks' main source of removals may be discards, and improvements in the accuracy of discard reporting would likely factor in more heavily than the precision of catches or composition sample sizes. Stocks in regions where fishery-independent surveys have covered the stock well may not show a large effect if the survey CVs were reduced. Also, we examined how improvements in the existing data would affect accuracy of the assessment. We did not look at the effect of adding a new data source, such as a survey, or expanding the spatial coverage of the fleets or surveys, though that is a future research goal.

Our input data were compiled using a number of reconstructed data sets (e.g. recreational landings before 1981), but we did not look into whether those methods of reconstruction had an effect on our results. We did this for two reasons: we were interested in the effect of improving data collection now, and the methods for historical reconstruction were too varied (ranging from linear interpolation between no catches and the first year of reported data to applying a catch rate to effort estimates back in time). Changing the uncertainty of those catches back in time would not address our main objective of informing current data collection efforts.

Steepness is difficult to estimate and is often fixed in practice. However, the contrast in our data likely allowed for the parameter to be estimated in a large proportion of our model runs (Conn et al. 2010). In the model runs that remained after we culled the bounded runs (as 
325 described in the Methods section) the estimates of steepness were consistently accurate. If our

326 327 estimable.

We did not attempt to address bias in any of the data sets. We have little information

329

330 amalgam species demonstrated a different exploitation pattern, steepness may not have been about which direction bias might occur in the actual data. For example, we only have speculative information about whether landings may be over or underreported, or whether discards are over or underestimated. It is known that an increase in sample size cannot overcome a known bias (Coggins and Quinn II 1998). However, without better evidence of the direction of a potential bias, we decided to focus our study on precision.

Finally, we did not initially incorporate aging error in our simulation. The assessments conducted in our region rarely show an effect when the aging error matrix is used for a sensitivity analysis. However, our results beg the question of whether substantial aging error would dampen our results, and change the data prioritization advice. Again we considered precision rather than bias, and ran alternative scenarios with an aging error matrix that was larger than any ever used for our region (i.e. the readers agreed only half the time) before we saw a change in the results. The amount of aging error normally reported for stocks in our region did not cause a change in the data prioritization advice. In the extreme scenario we had to use in order to perturb our initial results, one might argue that an age-structured model should be avoided if the aging is so uncertain. 
346

347

348

349

350

\section{Acknowledgements:}

We would like to thank the NOAA-Assessment Methods Working Group grant that funded this project. We appreciate the helpful comments and criticisms about the manuscript from Kevin Craig and Amy Schueller, and we thank the anonymous reviewers for their helpful suggestions.

\section{References}

Andrews, R., Brick, J.M., and Mathiowetz, N.A. 2014. Development and testing of recreational fishing effort surveys; Testing a mail survey design. Edited by NMFS. p. 56.

Charnov, E.L., Gislason, H., and Pope, J.G. 2013. Evolutionary assembly rules for fish life histories. Fish and Fisheries 14: 213-224.

Coggins, L.G., and Quinn II, T.J. 1998. A Simulation Study of the Effects of Aging Error Sample Size on Sustained Yield Estimates. In Fishery Stock Assessment Models. Edited by F. Funk, T.J. Quinn II, J. Heifetz, J.N. Ianelli, J.E. Powers, J.F. Schweighert, P.J. Sullivan and C.-I. Zhang. Alaska Sea Grant College - AK-SG-98-01, Anchorage, AK. pp. 955-976.

Conn, P.B., Williams, E.H., and Shertzer, K.W. 2010. When can we reliably estimate the productivity of fish stocks? Can J Fish Aquatic Sci 67(3): 511-523.

Fogarty, M.J., Sissenwine, M.P., and Cohen, E.B. 1991. Recruitment variability and the dynamics of exploited marine populations. TREE 6(8): 241-246.

Fournier, D.A., Skaug, H.J., Ancheta, J., Ianelli, J.N., Magnusson, A., Maunder, M.N., Nielsen, A., and Sibert, J.R. 2012. AD Model Builder: using automatic differentiation for statistical inference of highly parameterized complex nonlinear models. Optimization Methods and Software 27: 233-249. 
368

369

370

371

372

373

374

375

376

377

378

379

380

381

382

383

384

385

386

387

388

389

390

Magnusson, A., and Hilborn, R. 2007. What makes fisheries data informative? Fish and Fisheries 8(4): 337-358.

Maunder, M.N., and Punt, A.E. 2013. A review of integrated analysis in fisheries stock assessment. Fish Res 142(0): 61-74.

Methot, R.D. 2000. Technical description of the Stock Synthesis assessment program. NOAA Technical Memorandum. NMFS-NWFSC-43.

Powers, J.E., and Restrepo, V.R. 1993. Evaluation of Stock Assessment Research for Gulf of Mexico King Mackerel: Benefits and Costs to Management. North American Journal of Fisheries Management 13(1): 15-26.

Punt, A.E., Smith, D.C., and Smith, A.D.M. 2011. Among-stock comparisons for improving stock assessments of data-poor stocks: the "Robin Hood" approach. ICES Journal of Marine Science: Journal du Conseil 68(5): 972-981.

Rindone, R.R., Kellison, G.T., and Bortone, S.A. 2015. Data availability for Red Snapper in Gulf of Mexico and southeastern U.S. Atlantic ocean waters, North American Journal of Fisheries Management 35(2): 191-204.

SEDAR. 2008. SEDAR 15 - Stock Assessment Report 2 (SAR 2) South Atlantic Greater Amberjack, North Charleston, SC.

SEDAR. 2009. SEDAR 19 - South Atlantic Red Grouper Stock Assessment Report, North Charleston, SC.

SEDAR. 2010. SEDAR 24 - South Atlantic Red Snapper Stock Assessment Report, North Charleston, SC.

SEDAR. 2012a. SEDAR 1 Update - Stock Assessment of Red Porgy off the Southeastern United States, North Charleston, SC. 
391 SEDAR. 2012b. SEDAR 17 Update - Stock Assessment of Vermilion Snapper off the 392 Southeastern United States, North Charleston, SC.

393 SEDAR. 2013a. SEDAR 25 Update - Stock Assessment of Black Sea Bass off the Southeastern $394 \quad$ United States, North Charleston, SC.

395 SEDAR. 2013b. SEDAR 36 - South Atlantic Snowy Grouper Stock Assessment Report, North $396 \quad$ Charleston, SC.

397 SEDAR. 2014. SEDAR 10 Update - Stock Assessment of Gag off the Southeastern United States, North Charleston, SC.

399 Szuwalski, C., and Punt, A.E. 2012. Identifying research priorities for management under uncertainty: The estimation ability of the stock assessment method for eastern Bering Sea snow crab (Chionoecetes opilio). Fish Res 134-136: 82-94.

402

von Bertalanffy, L. 1938. A quantitative theory of organic growth (inquiries on growth laws. II). Human Biology 10(2): 181-213.

Williams, E.H., and Shertzer, K.W. 2015. Technical documentation of the Beaufort Assessment Model (BAM). U.S. Department of Commerce, NOAA Technical Memorandum NMFSSEFSC-671. 43 p. doi:10.7289/V57M05W6

Yin, Y., and Sampson, D.B. 2004. Bias and Precision of Estimates from an Age-Structured Stock Journal of Fisheries Management 24(3): 865-879. 
Figure Legends

Fig. 1. The von Bertalanffy growth curve for the amalgam species plotted with the curves for each of the species included in the amalgam.

Fig. 2. Recreational CPUE and survey index used in the base model run. The points represent the model inputs and the grey error bars are the $5^{\text {th }}$ and $95^{\text {th }}$ quantiles of the model fits to the data.

Fig. 3. Proportion of total fishing mortality attributed to commercial landings, recreational landings, commercial discards, and recreational discards used in the base model. Each of the eight stocks is plotted in grey with the averaged trend over all the stocks in black.

Fig. 4. Total fishing mortality applied to the stock in the base model (shown in black). Each of the eight stocks used to inform the average trend are shown in grey for reference.

Fig. 5. Proportional selectivity by age for each fleet and survey. The commercial, recreational, and survey selectivities are each means of the various assessment model fits from the eight species. The discard selectivity is for both the commercial and recreational fleet.

Fig. 6. Model fits to the recreational (column one) and commercial (column two) landings (row one) and discards (row two). The points represent the model inputs and the grey error bars are the $5^{\text {th }}$ and $95^{\text {th }}$ quantiles of the model fits to the data. 
Fig. 7. Estimates of $\mathrm{F} / \mathrm{F}_{\mathrm{MSY}}, \mathrm{SSB} / \mathrm{SSB}_{\mathrm{MSY}}$, and $\mathrm{ABC}(\mathrm{klb})$ plotted alongside the relative error of the base data (solid line) and the improved data (dashed line) assessments. The vertical lines are medians of the bootstrap replicates. 
Table 1. The compiled parameters for the amalgam species. These parameters represent species in the Atlantic waters of the southeast U.S.: Vermilion Snapper (VS), Red Porgy (RP), Black Sea Bass (BSB), Red Snapper (RS), Greater Amberjack (GAJ), Red Grouper (RG), Snowy Grouper (SG), and Gag (GAG). AVG represents the value used in the simulated population. In most cases, we used an arithmetic mean. However, we excluded GAJ growth parameters, as they seemed to deviate from the snapper/grouper complex. MSST is the minimum stock size threshold, which is used to determine whether a stock is considered overfished.

\begin{tabular}{|c|c|c|c|c|c|c|c|c|c|}
\hline & VS & RP & BSB & GAJ & RS & RG & GAG & SG & AVG \\
\hline Start year & 1946 & 1972 & 1978 & 1946 & 1955 & 1976 & 1962 & 1974 & 1963 \\
\hline $\mathrm{L}_{\infty}$ & 506 & 528 & 496 & 1194 & 902 & 848 & 1091 & 1065 & 792 \\
\hline $\mathrm{k}$ & 0.12 & 0.20 & 0.18 & 0.34 & 0.24 & 0.21 & 0.17 & 0.09 & 0.18 \\
\hline $\mathrm{t}_{0}$ & -3.50 & -1.53 & -0.92 & -0.45 & -0.03 & -0.67 & -1.31 & -2.88 & -1.36 \\
\hline Steepness & 0.71 & 0.41 & 0.48 & 0.74 & 0.85 & 0.92 & 0.95 & 0.84 & 0.74 \\
\hline $\mathrm{M}_{\text {MSsT }}$ & 0.22 & 0.225 & 0.38 & 0.23 & 0.08 & 0.14 & 0.115 & 0.12 & 0.19 \\
\hline $\mathrm{A}_{\text {mat }}$ & 1 & 1 & 1 & 1 & 2 & 3 & 3 & 6 & 2.25 \\
\hline $\begin{array}{c}\text { Max age } \\
\text { Years of }\end{array}$ & 12 & 14 & 11 & 10 & 20 & 16 & 20 & 25 & 16 \\
$\begin{array}{c}\text { Commercial } \\
\text { comps }\end{array}$ & 19 & 13 & 12 & 7 & 8 & 5 & 15 & 14 & 12 \\
\hline $\begin{array}{c}\text { Years of } \\
\text { Recreational } \\
\text { comps }\end{array}$ & 27 & 8 & 11 & 1 & 20 & 11 & 12 & 3 & 12 \\
\hline $\begin{array}{c}\text { Years of } \\
\text { Survey } \\
\text { comps }\end{array}$ & 10 & 22 & 23 & 0 & 0 & 11 & 0 & 5 & 9 \\
\hline
\end{tabular}


Table 2. Scenarios considered for each of the data improvements. The rows are each model run, either the base model or a run named after the data source that was improved. The columns are either CVs (in the case of indices, landings, or discards) or sample size (in the case of composition data). The base case contains no data improvements and each data improvement across columns is in bold and denoted with an asterisk.

\begin{tabular}{|c|c|c|c|c|c|c|c|c|c|}
\hline & $\begin{array}{l}\text { Survey } \\
\text { CPUE CV }\end{array}$ & $\begin{array}{c}\text { Survey } \\
\text { comps } \\
\text { sample } \\
\text { size }\end{array}$ & $\begin{array}{l}\text { Recreational } \\
\text { landings CV }\end{array}$ & $\begin{array}{l}\text { Recreational } \\
\text { discards CV }\end{array}$ & $\begin{array}{l}\text { Recreational } \\
\text { CPUE CV }\end{array}$ & $\begin{array}{l}\text { Recreational } \\
\text { comps } \\
\text { sample size }\end{array}$ & $\begin{array}{l}\text { Commercial } \\
\text { landings CV }\end{array}$ & $\begin{array}{l}\text { Commercial } \\
\text { discards CV }\end{array}$ & $\begin{array}{c}\text { Commercial } \\
\text { comps } \\
\text { sample size }\end{array}$ \\
\hline Base & 0.75 & 25 & 0.75 & 1 & 0.5 & 10 & 0.05 & 1 & 30 \\
\hline $\begin{array}{c}\text { Commercial } \\
\text { comps sample } \\
\text { size }\end{array}$ & 0.75 & 25 & 0.75 & 1 & 0.5 & 10 & 0.05 & 1 & $150 *$ \\
\hline $\begin{array}{l}\text { Commercial } \\
\text { discards CV }\end{array}$ & 0.75 & 25 & 0.75 & 1 & 0.5 & 10 & 0.05 & $0.25 *$ & 30 \\
\hline $\begin{array}{c}\text { Recreational } \\
\text { comps sample } \\
\text { size }\end{array}$ & 0.75 & 25 & 0.75 & 1 & 0.5 & $50 *$ & 0.05 & 1 & 30 \\
\hline $\begin{array}{l}\text { Recreational } \\
\text { CPUE CV }\end{array}$ & 0.75 & 25 & 0.75 & 1 & $0.125 *$ & 10 & 0.05 & 1 & 30 \\
\hline $\begin{array}{l}\text { Recreational } \\
\text { discards CV }\end{array}$ & 0.75 & 25 & 0.75 & $0.25 *$ & 0.5 & 10 & 0.05 & 1 & 30 \\
\hline $\begin{array}{l}\text { Recreational } \\
\text { landings CV }\end{array}$ & 0.75 & 25 & 0.1875* & 1 & 0.5 & 10 & 0.05 & 1 & 30 \\
\hline $\begin{array}{c}\text { Survey } \\
\text { CPUE CV }\end{array}$ & $0.1875 *$ & 25 & 0.75 & 1 & 0.5 & 10 & 0.05 & 1 & 30 \\
\hline $\begin{array}{c}\text { Survey } \\
\text { comps sample } \\
\text { size }\end{array}$ & 0.75 & $125 *$ & 0.75 & 1 & 0.5 & 10 & 0.05 & 1 & 30 \\
\hline $\begin{array}{l}\text { Survey CPUE } \\
\mathrm{CV}+\text { comps } \\
\text { sample size }\end{array}$ & $0.1875^{*}$ & $125 *$ & 0.75 & 1 & 0.5 & 10 & 0.05 & 1 & 30 \\
\hline $\begin{array}{c}\text { Commercial } \\
\text { discards CV + } \\
\text { comps sample } \\
\text { size } \\
\end{array}$ & 0.75 & 25 & 0.75 & 1 & 0.5 & 10 & 0.05 & $0.25 *$ & $150 *$ \\
\hline $\begin{array}{l}\text { All recreational } \\
\text { components }\end{array}$ & 0.75 & 25 & $0.1875 *$ & $0.25 *$ & $0.125 *$ & $50 *$ & 0.05 & 1 & 30 \\
\hline $\begin{array}{c}\text { All } \\
\text { comps sample } \\
\text { sizes } \\
\end{array}$ & 0.75 & $125 *$ & 0.75 & 1 & 0.5 & $50 *$ & 0.05 & 1 & $150 *$ \\
\hline $\begin{array}{c}\text { All } \\
\text { indices CVs }\end{array}$ & $0.1875 *$ & 25 & $0.1875^{*}$ & 1 & $0.125 *$ & 10 & 0.05 & 1 & 30 \\
\hline $\begin{array}{c}\text { Rec landings CV } \\
\text { + all discards } \\
\text { CVs }\end{array}$ & 0.75 & 25 & $0.1875 *$ & $0.25 *$ & 0.5 & 10 & 0.05 & $0.25 *$ & 30 \\
\hline
\end{tabular}


Table 3. The mean relative errors of the base assessment and each assessment of an improved data set. The values and category names in bold are the components or categories that contributed most to the accuracy of the assessment. The top section is the grouped data improvements, and the bottom contains the individual data component improvements. Culled is the proportion of runs culled due to steepness hitting the upper bound.

\begin{tabular}{|c|c|c|c|c|c|c|c|c|c|}
\hline & Steepness & Culled & $\mathrm{R}_{0}$ & $\mathrm{ABC}$ & MSY & $\mathrm{F}_{\mathrm{msy}}$ & $\mathrm{F} / \mathrm{F}_{\mathrm{msy}}$ & $\mathrm{SSB}_{\text {msy }}$ & $\mathrm{SSB} / \mathrm{SSB}_{\mathrm{msy}}$ \\
\hline Base & -0.01 & 0.116 & -0.32 & 0.05 & -0.22 & -0.15 & -0.67 & -0.35 & 0.38 \\
\hline $\begin{array}{c}\text { All } \\
\text { commercial }\end{array}$ & -0.01 & 0.095 & -0.28 & -0.01 & -0.21 & -0.11 & -0.37 & -0.31 & 0.29 \\
\hline $\begin{array}{c}\text { All } \\
\text { recreational }\end{array}$ & -0.01 & 0.102 & -0.29 & 0.01 & -0.22 & -0.11 & -0.48 & -0.32 & 0.35 \\
\hline $\begin{array}{l}\text { Landings and } \\
\text { discards }\end{array}$ & $\mathbf{0}$ & 0.113 & -0.32 & 0.05 & -0.23 & -0.12 & -0.72 & -0.36 & 0.39 \\
\hline $\begin{array}{l}\text { All survey } \\
\text { components }\end{array}$ & -0.01 & 0.075 & -0.28 & -0.06 & -0.22 & -0.09 & -0.2 & -0.31 & 0.24 \\
\hline All indices & -0.02 & 0.114 & -0.26 & 0.05 & -0.19 & -0.17 & -0.58 & -0.27 & 0.36 \\
\hline All comps & -0.01 & 0.088 & -0.22 & -0.03 & -0.17 & -0.1 & -0.28 & -0.23 & 0.24 \\
\hline $\begin{array}{l}\text { Recreational } \\
\text { age comps }\end{array}$ & $\mathbf{0}$ & 0.111 & -0.28 & 0.02 & -0.2 & -0.13 & -0.53 & -0.32 & 0.35 \\
\hline $\begin{array}{l}\text { Survey age } \\
\text { comps }\end{array}$ & -0.01 & 0.107 & -0.31 & -0.02 & -0.23 & -0.11 & -0.41 & -0.34 & 0.33 \\
\hline $\begin{array}{c}\text { Commercial } \\
\text { age comps }\end{array}$ & -0.01 & 0.100 & -0.28 & -0.02 & -0.21 & -0.12 & -0.36 & -0.30 & 0.29 \\
\hline $\begin{array}{l}\text { Recreational } \\
\text { index }\end{array}$ & -0.02 & 0.125 & -0.29 & 0.05 & -0.21 & -0.16 & -0.58 & -0.31 & 0.38 \\
\hline Survey index & $\mathbf{0}$ & 0.110 & -0.31 & 0.06 & -0.22 & -0.14 & -0.65 & -0.35 & 0.4 \\
\hline $\begin{array}{l}\text { Recreational } \\
\text { landings }\end{array}$ & -0.01 & 0.120 & -0.3 & 0.06 & -0.2 & -0.15 & -0.69 & -0.34 & 0.39 \\
\hline $\begin{array}{c}\text { Commercial } \\
\text { discards }\end{array}$ & -0.01 & 0.123 & -0.31 & 0.05 & -0.22 & -0.14 & -0.65 & -0.35 & 0.38 \\
\hline $\begin{array}{c}\text { Recreational } \\
\text { discards }\end{array}$ & -0.01 & 0.114 & -0.3 & 0.05 & -0.22 & -0.13 & -0.34 & -0.69 & 0.38 \\
\hline
\end{tabular}


1

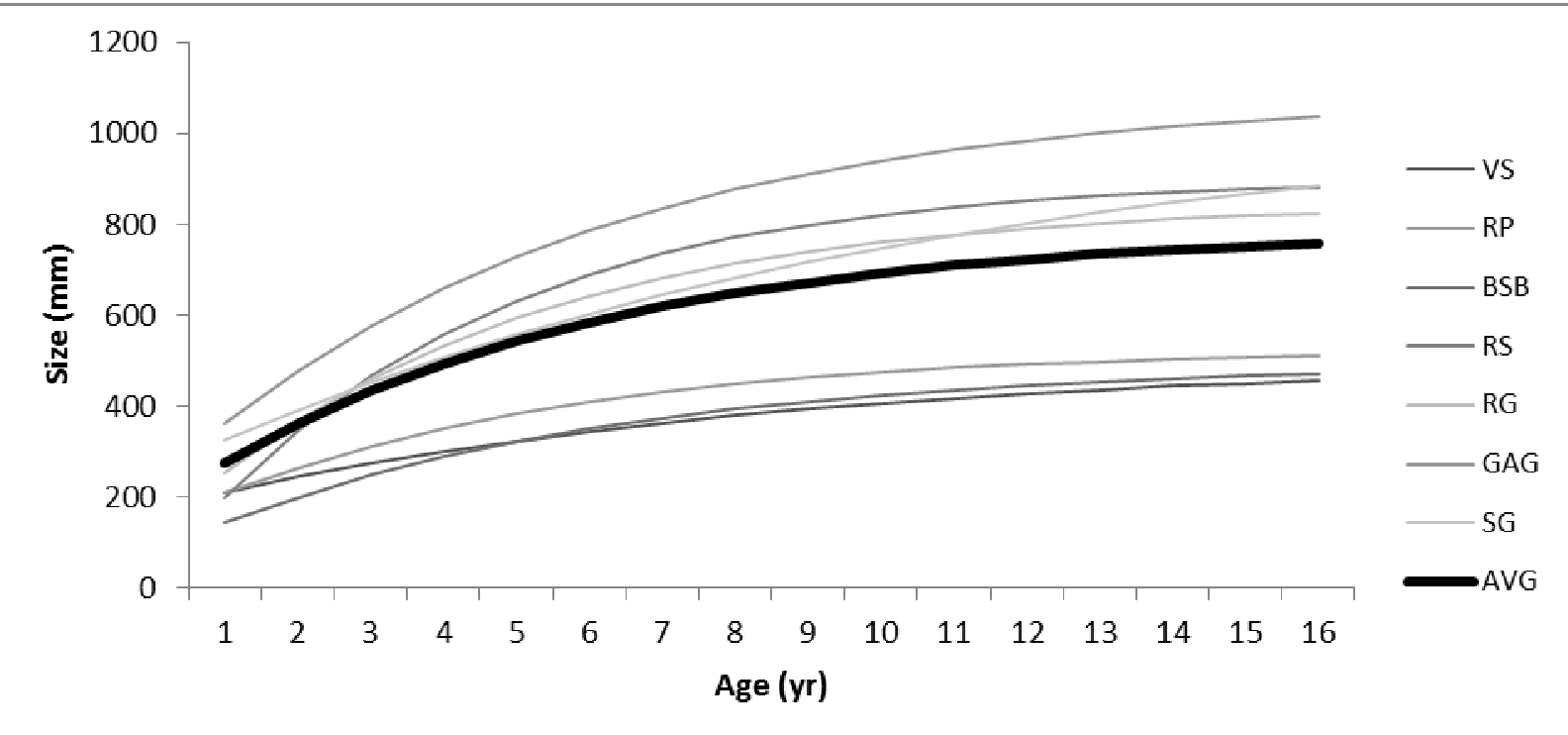

2

3

4

5

6

7

8

9

10 

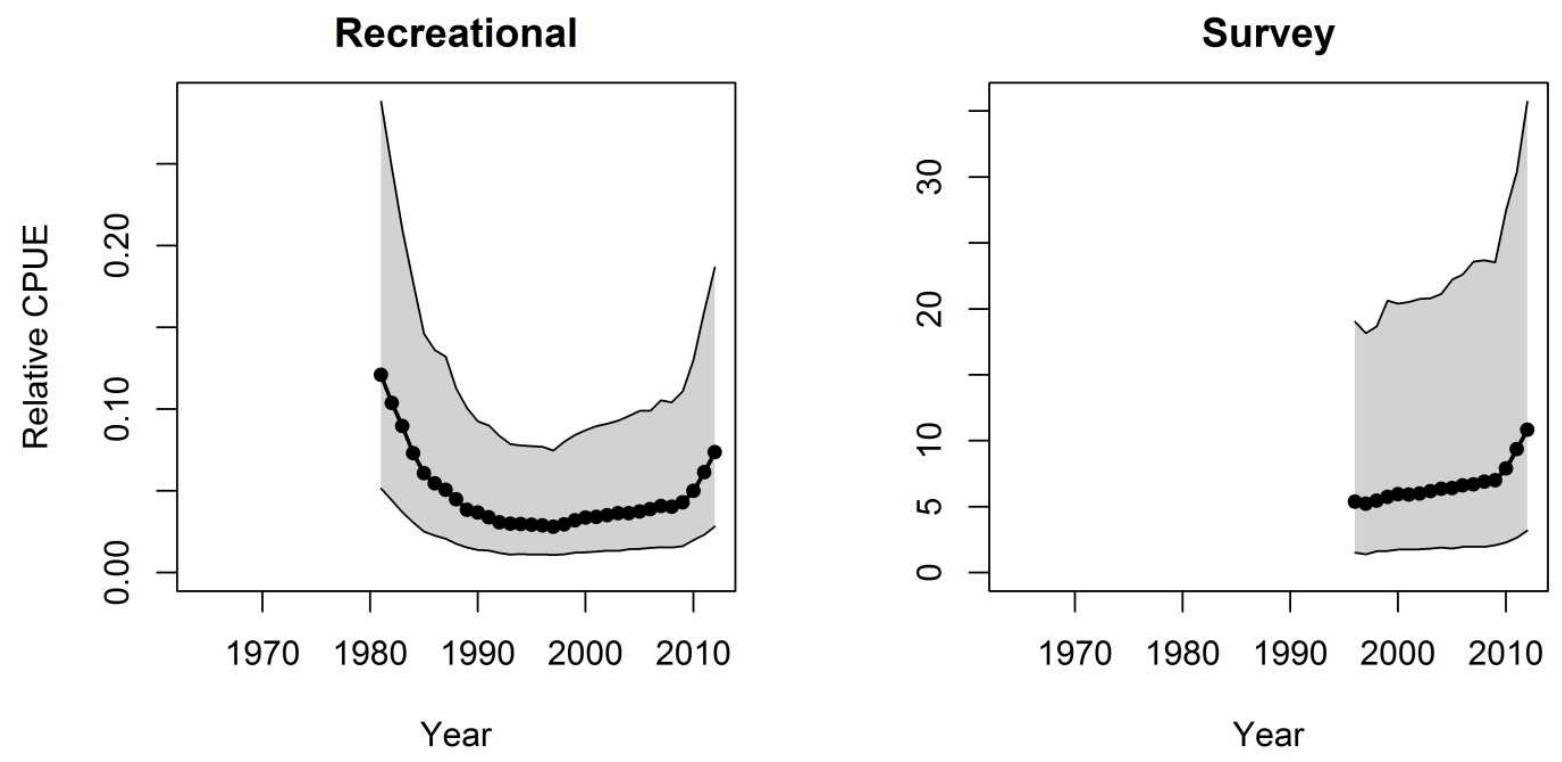

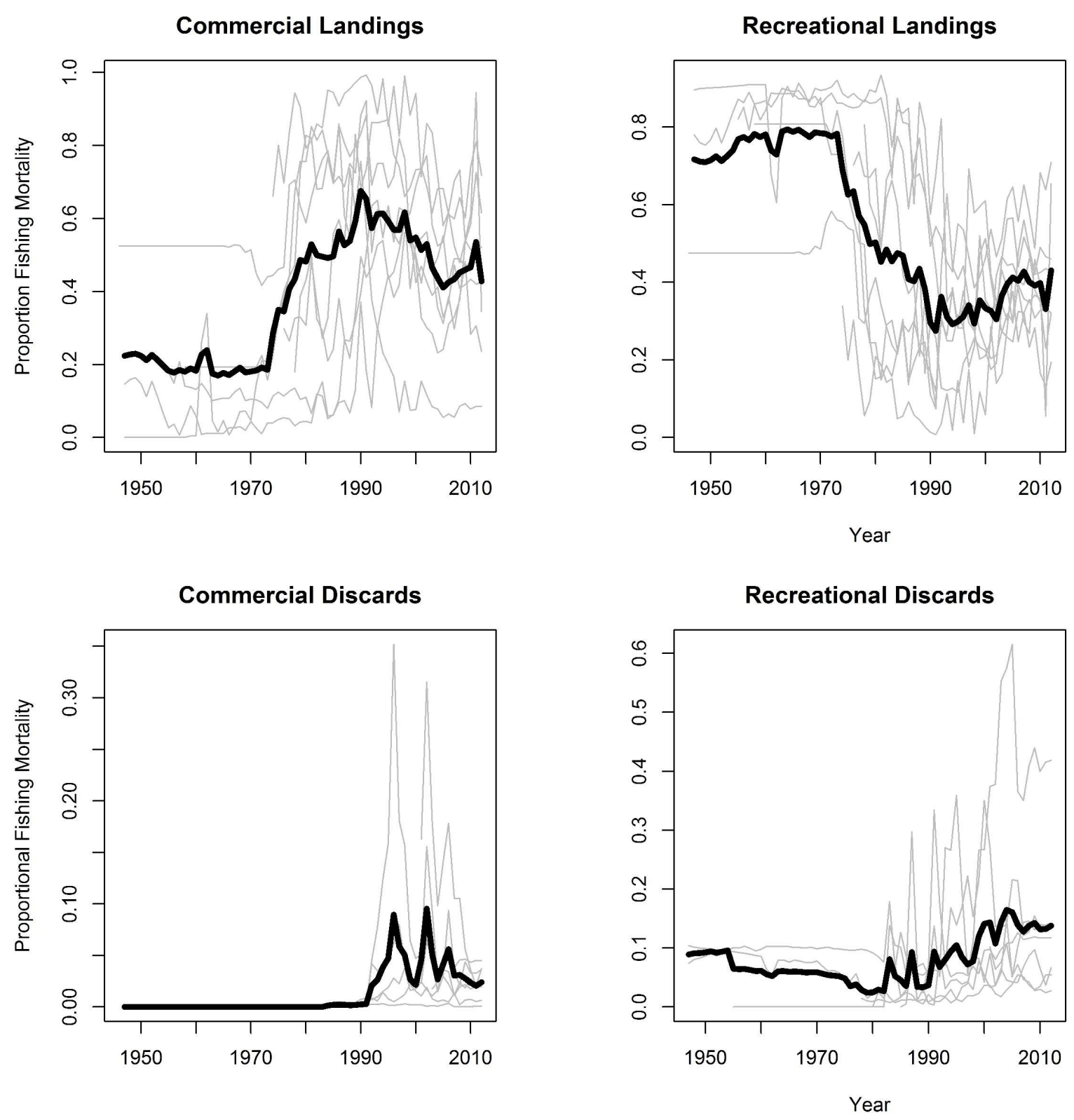


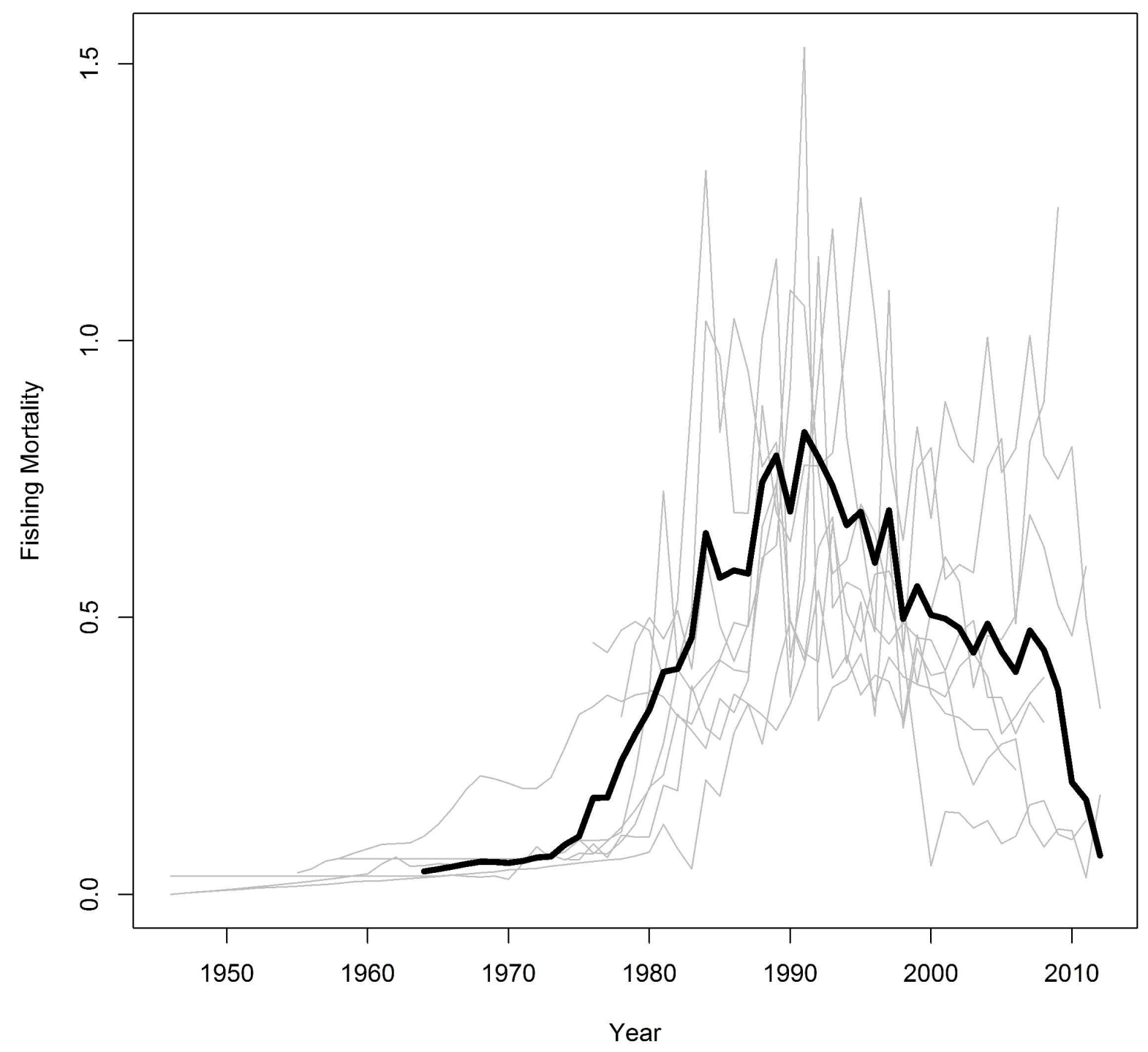




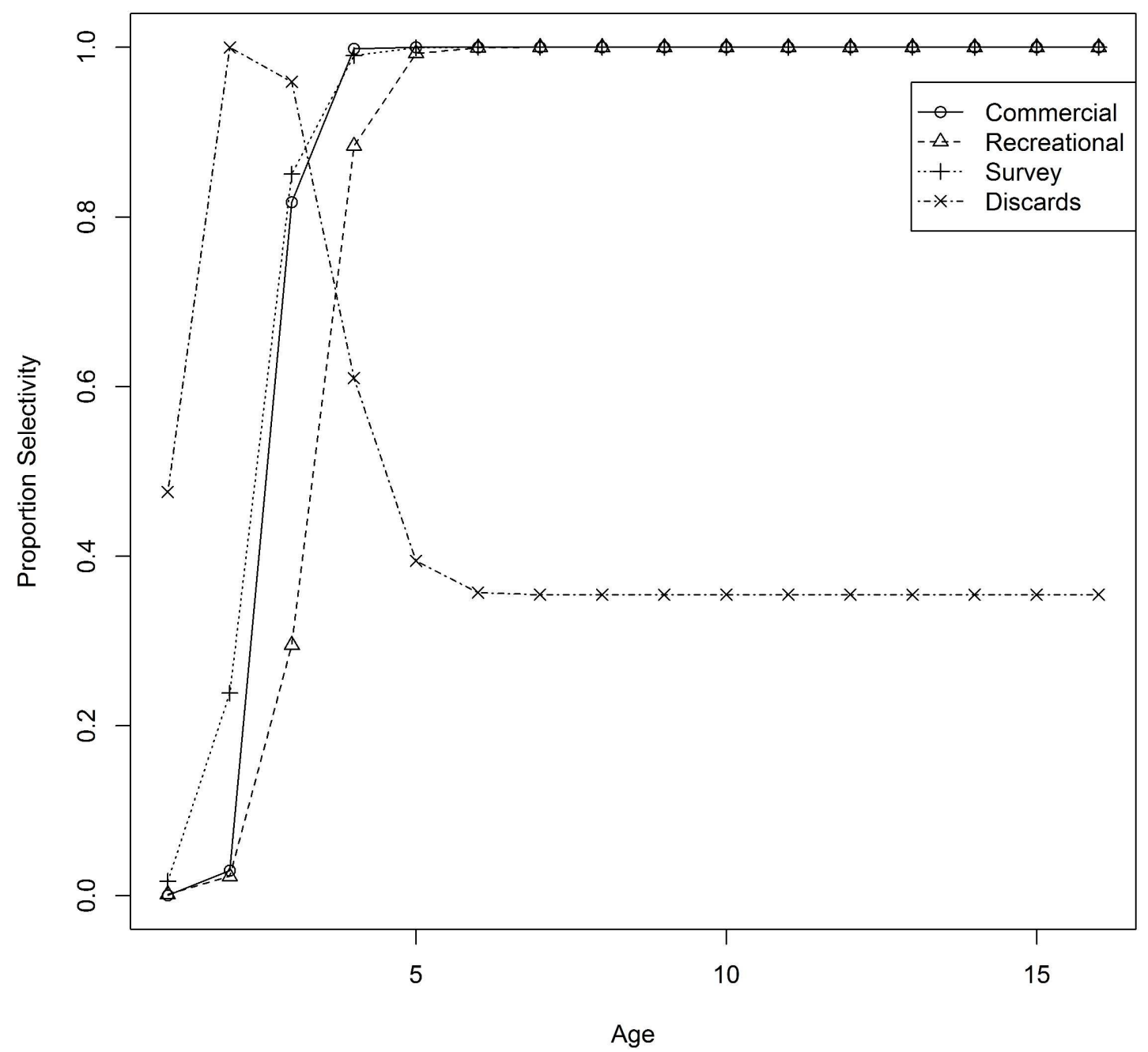

https://mc06.manuscriptcentral.com/cjfas-pubs 


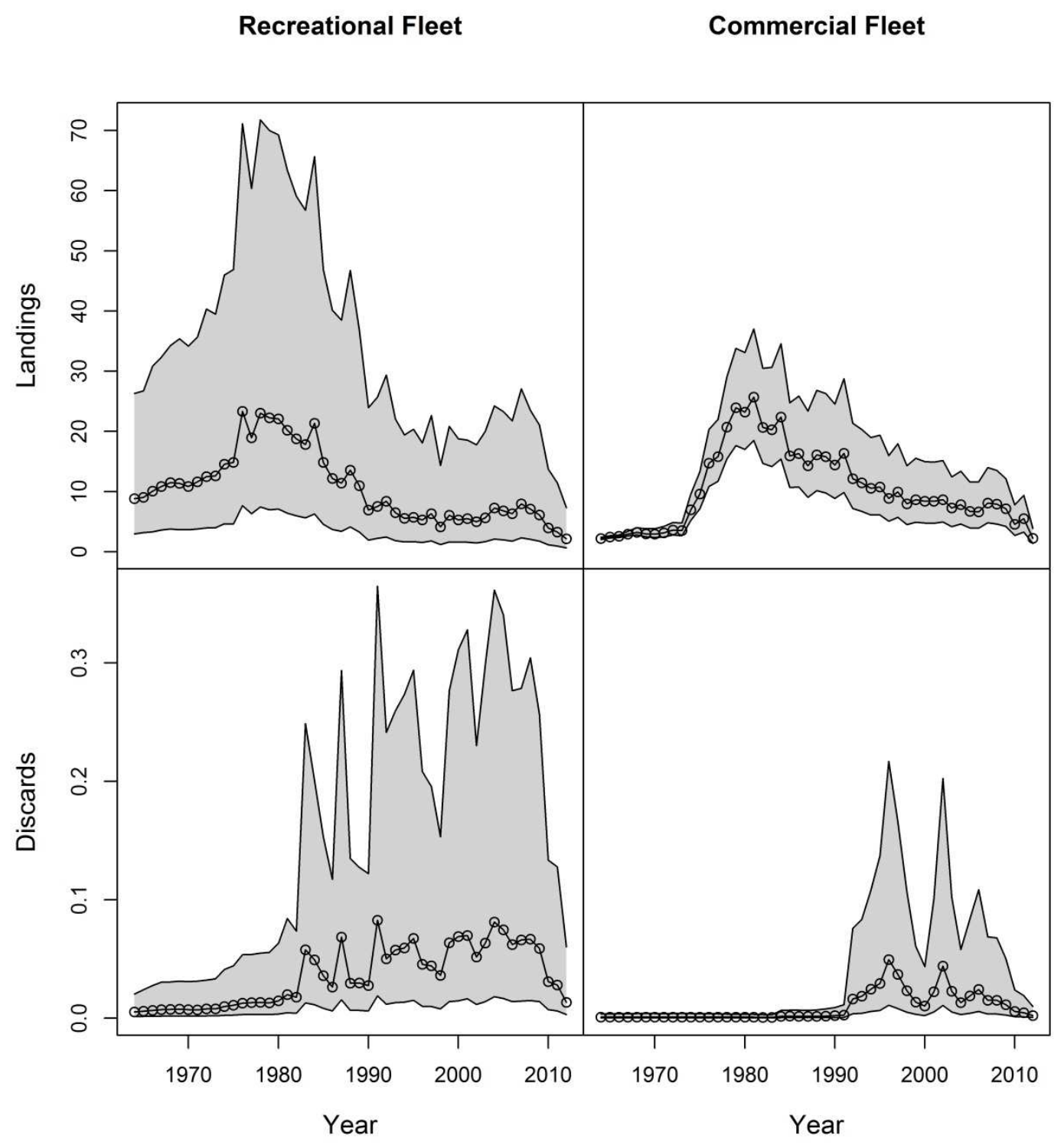



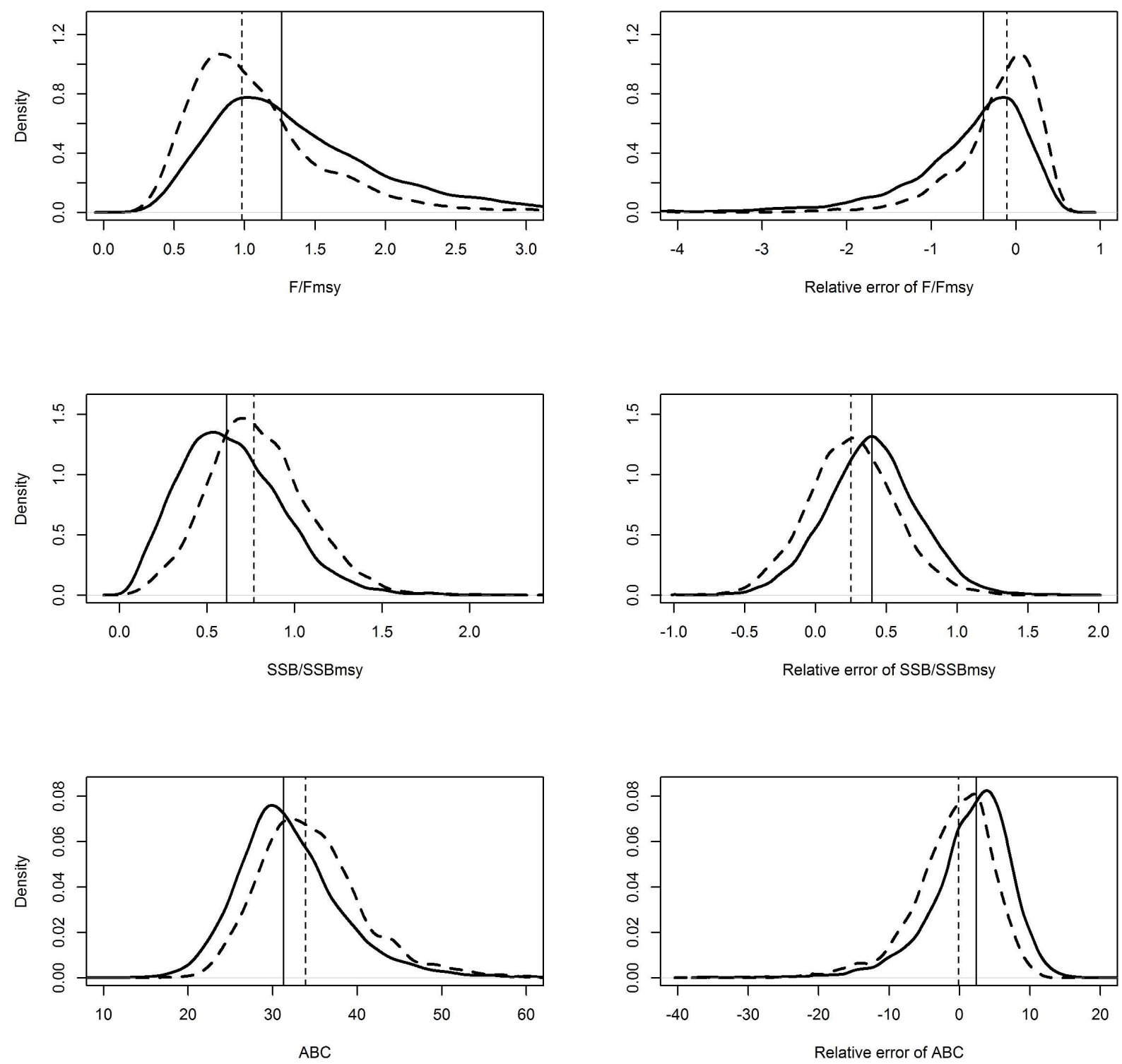
Supplementary Material

Figure S1. Commercial age compositions (increased sample size)
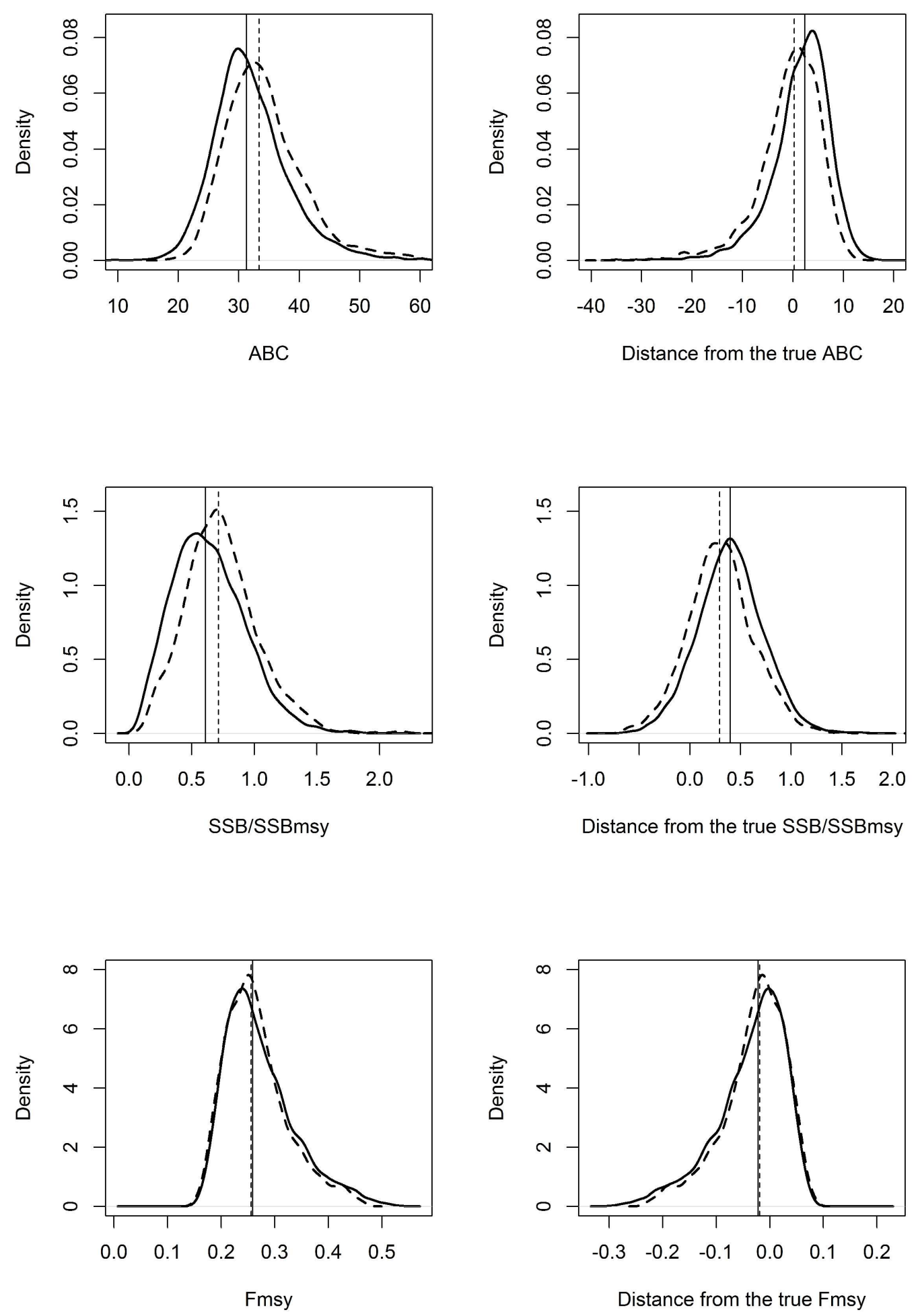
Supplementary Material

Figure S2. Commercial age compositions (increased sample size)
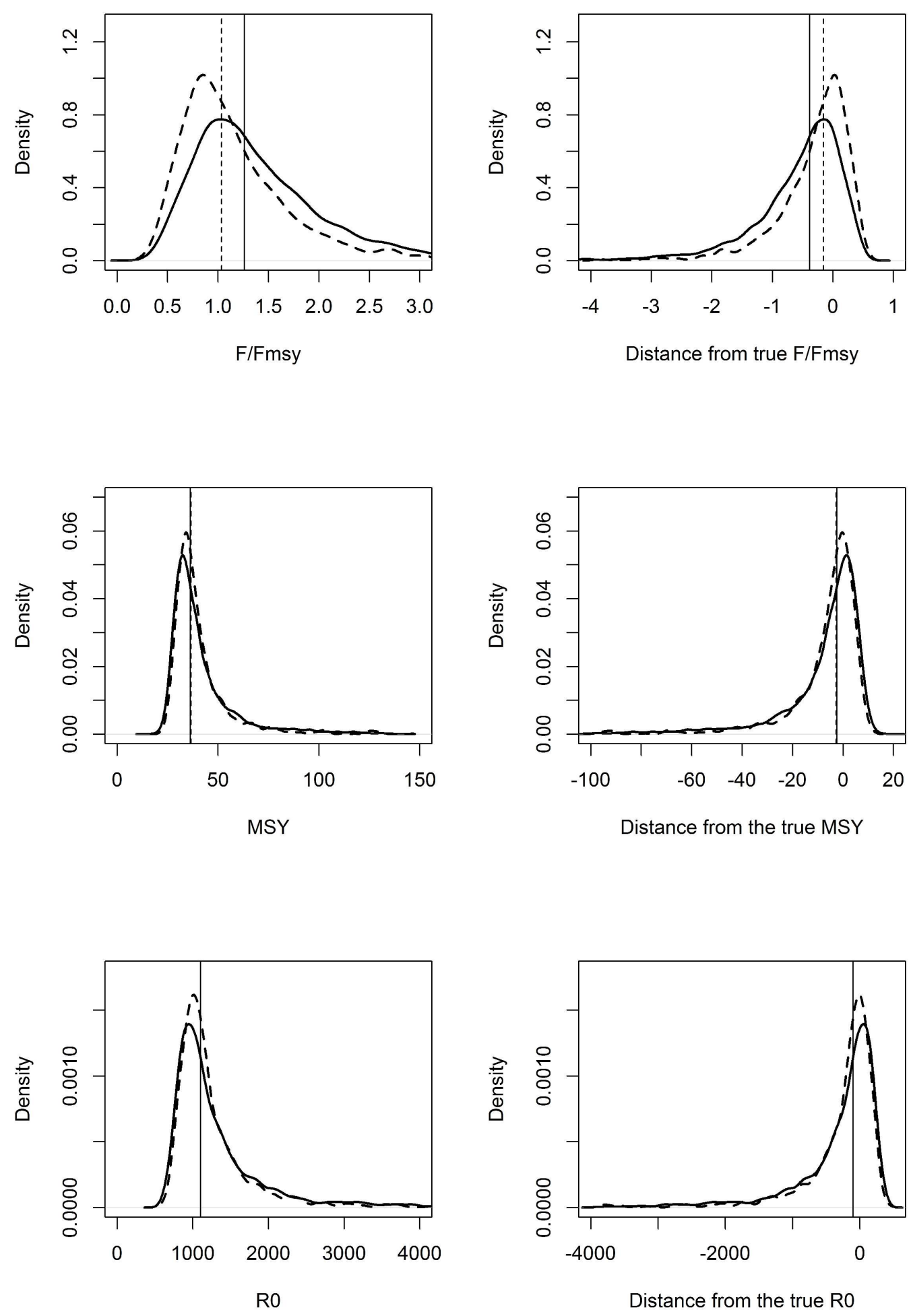
Supplementary Material

Figure S3. Commercial age compositions (increased sample size)
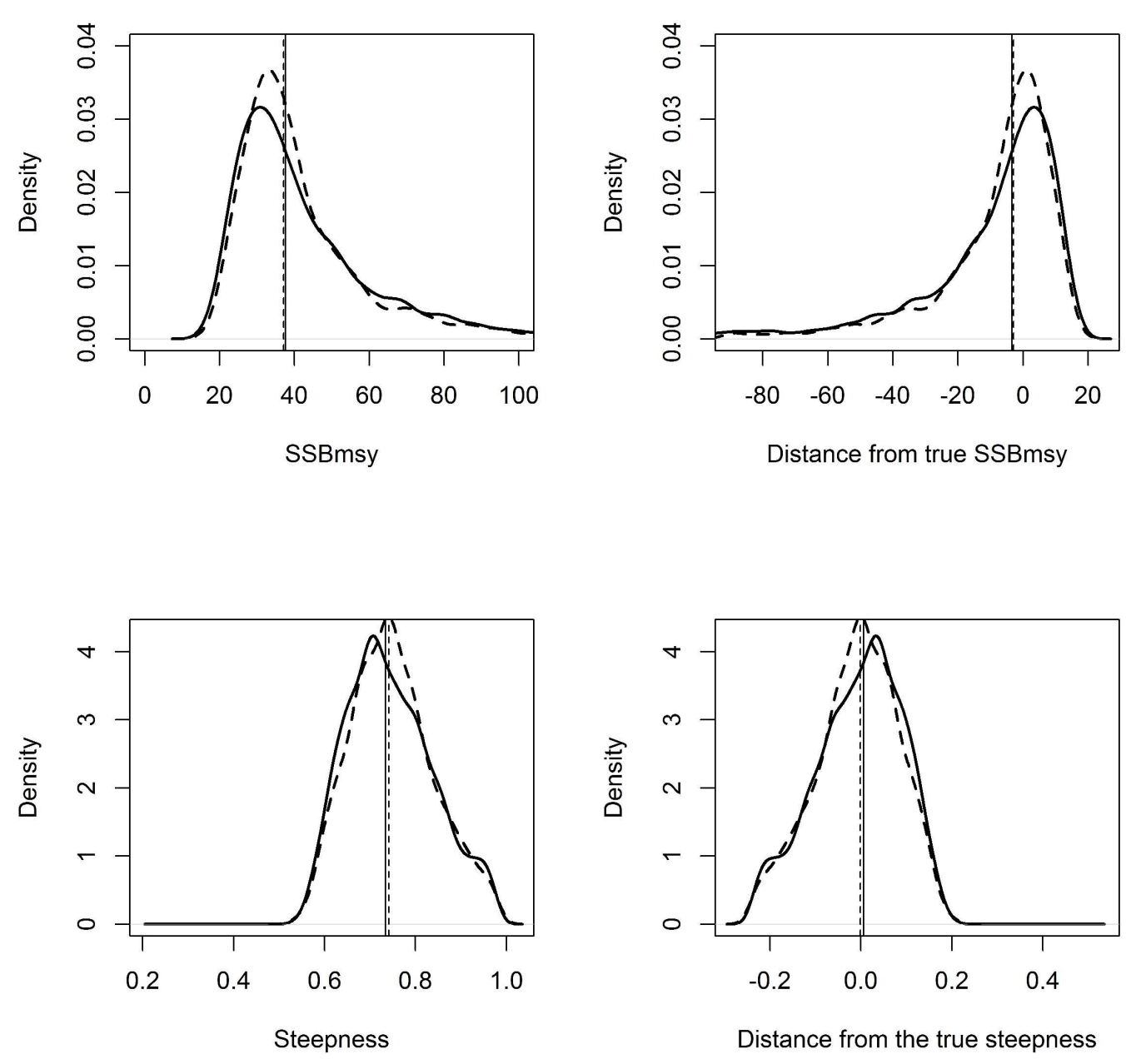
Supplementary Material

Figure S4. Commercial discards
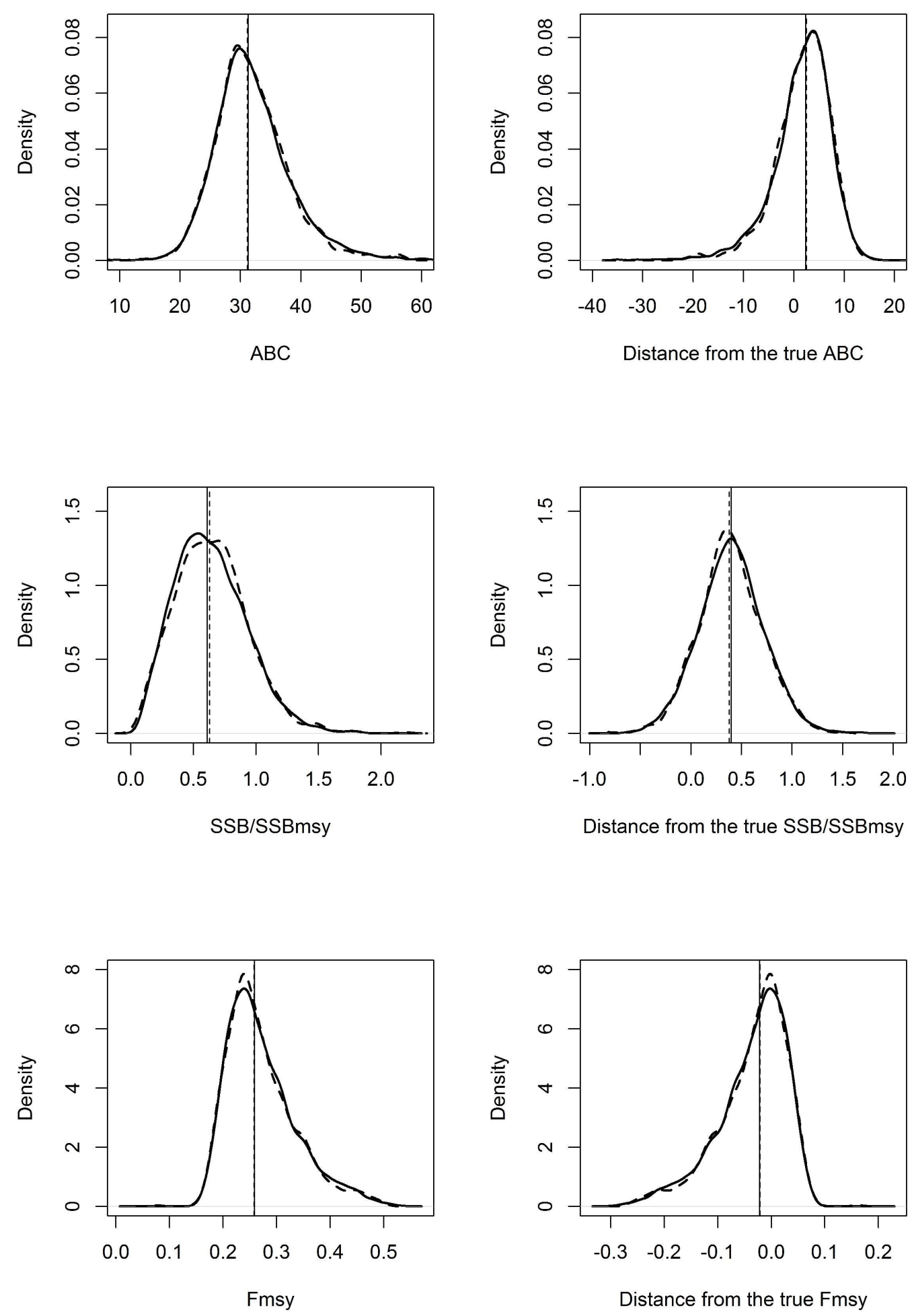
Supplementary Material

Figure S5. Commercial discards
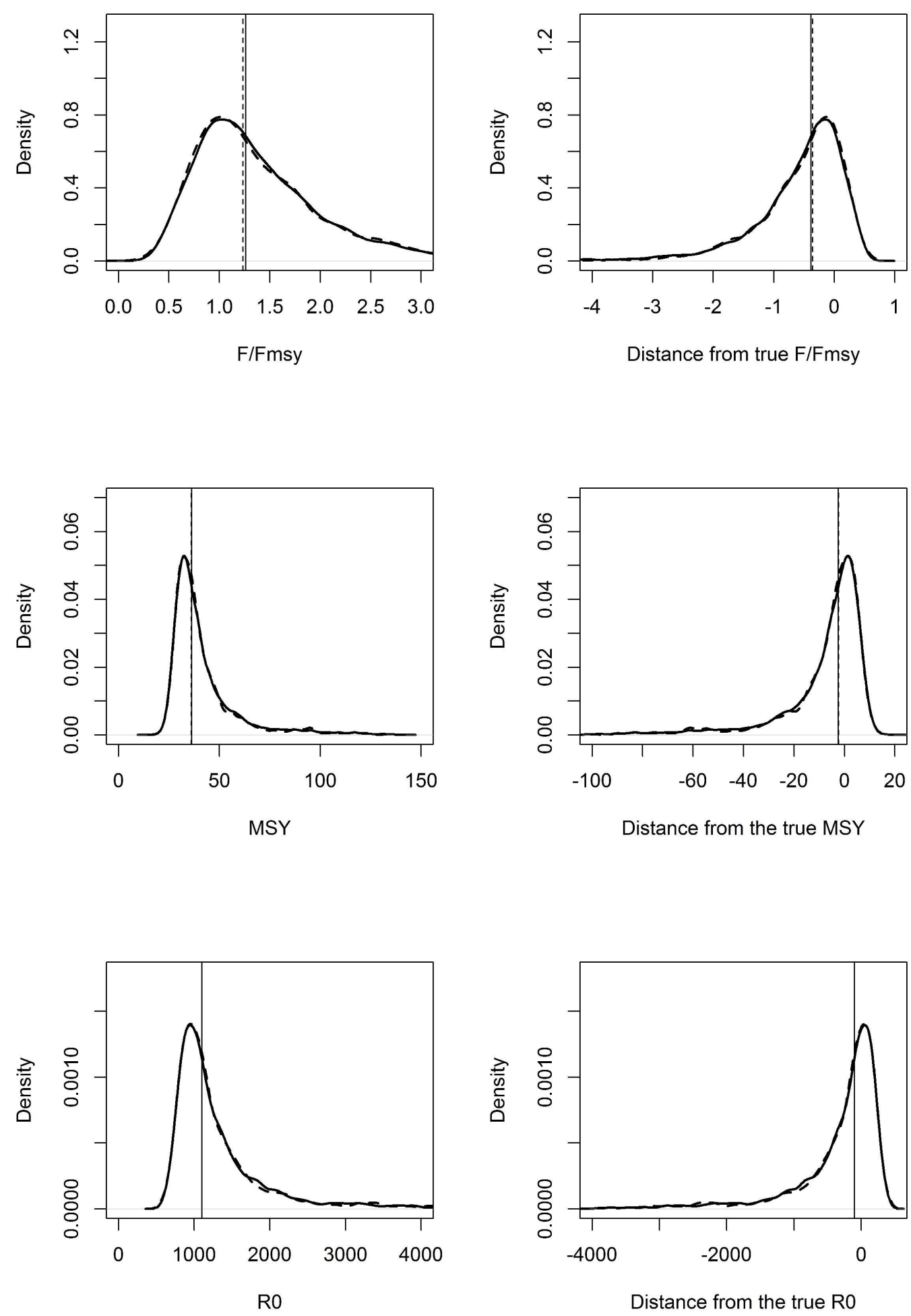
Supplementary Material

Figure S6. Commercial discards
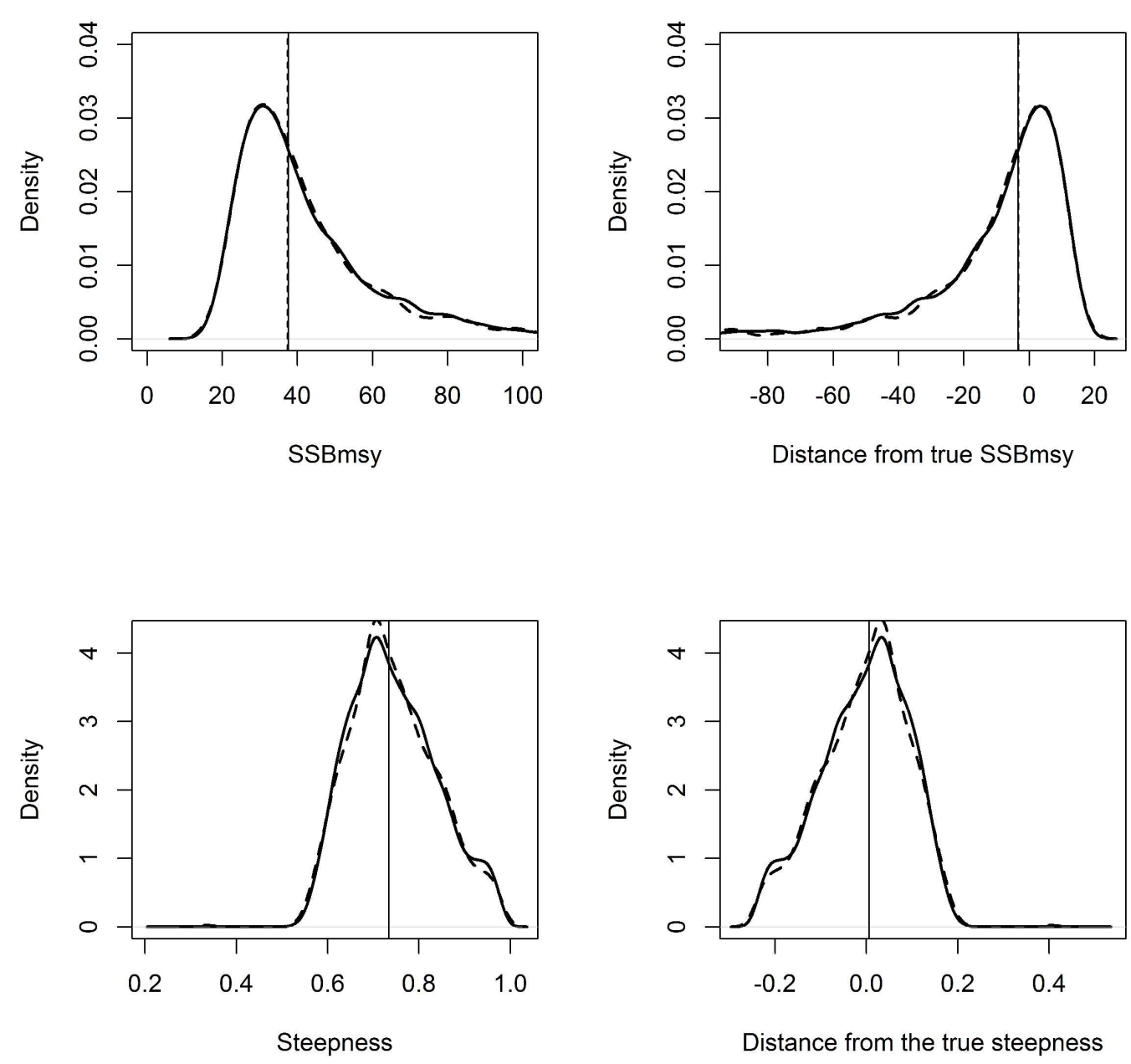

Distance from the true steepness 
Supplementary Material

Figure S7. Recreational age compositions (increased sample size)
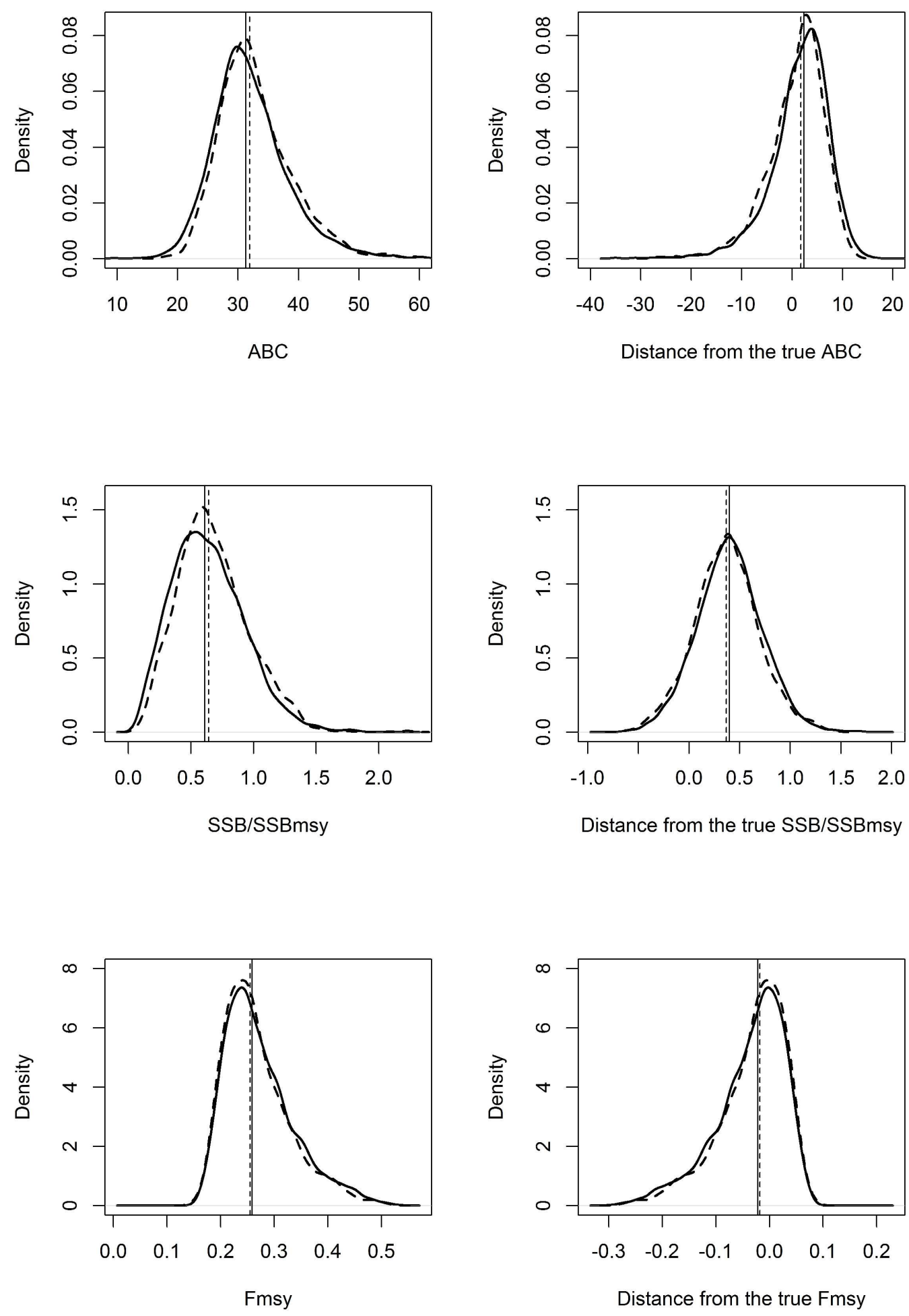
Supplementary Material

Figure S8. Recreational age compositions (increased sample size)
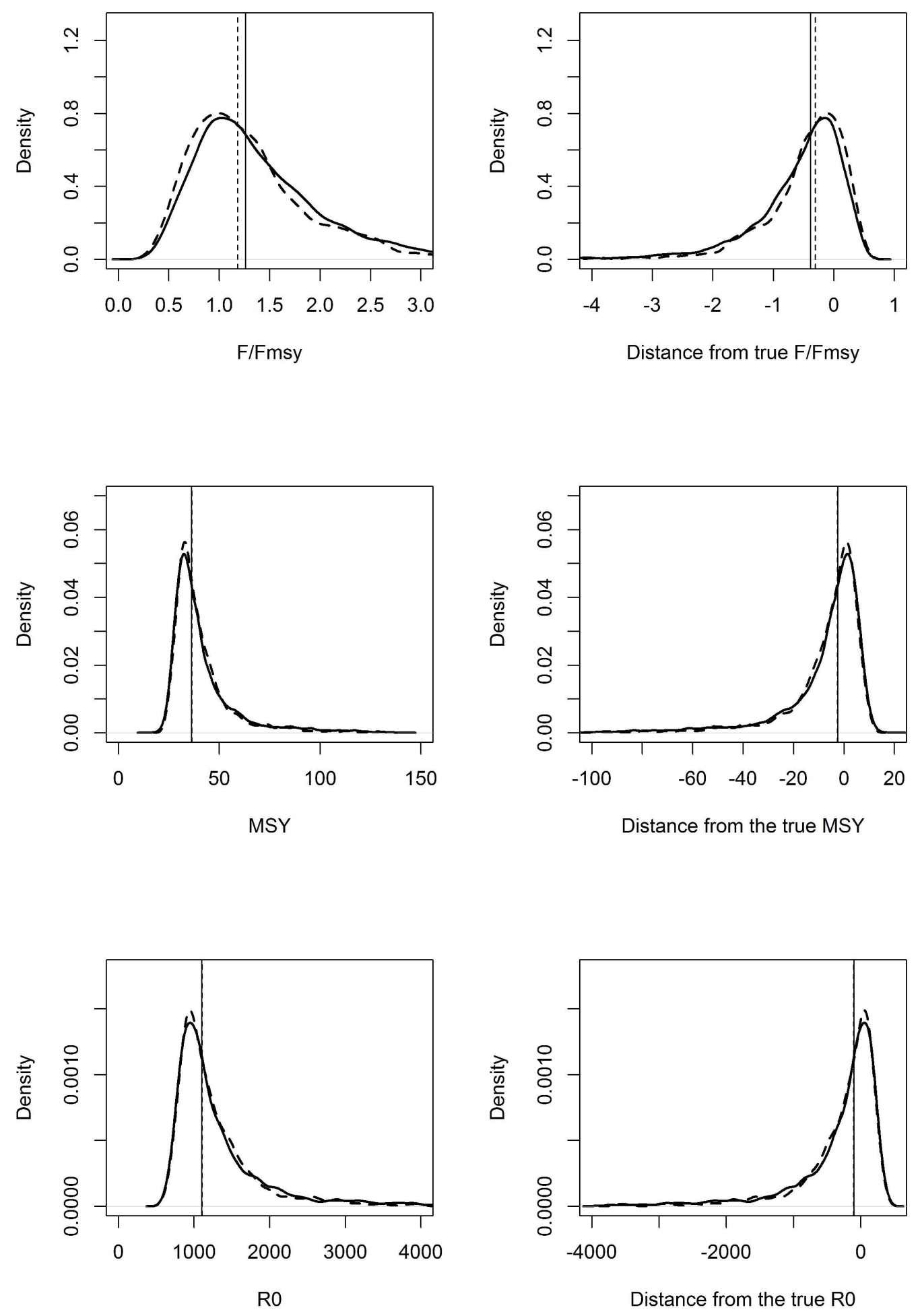
Supplementary Material

Figure S9. Recreational age compositions (increased sample size)
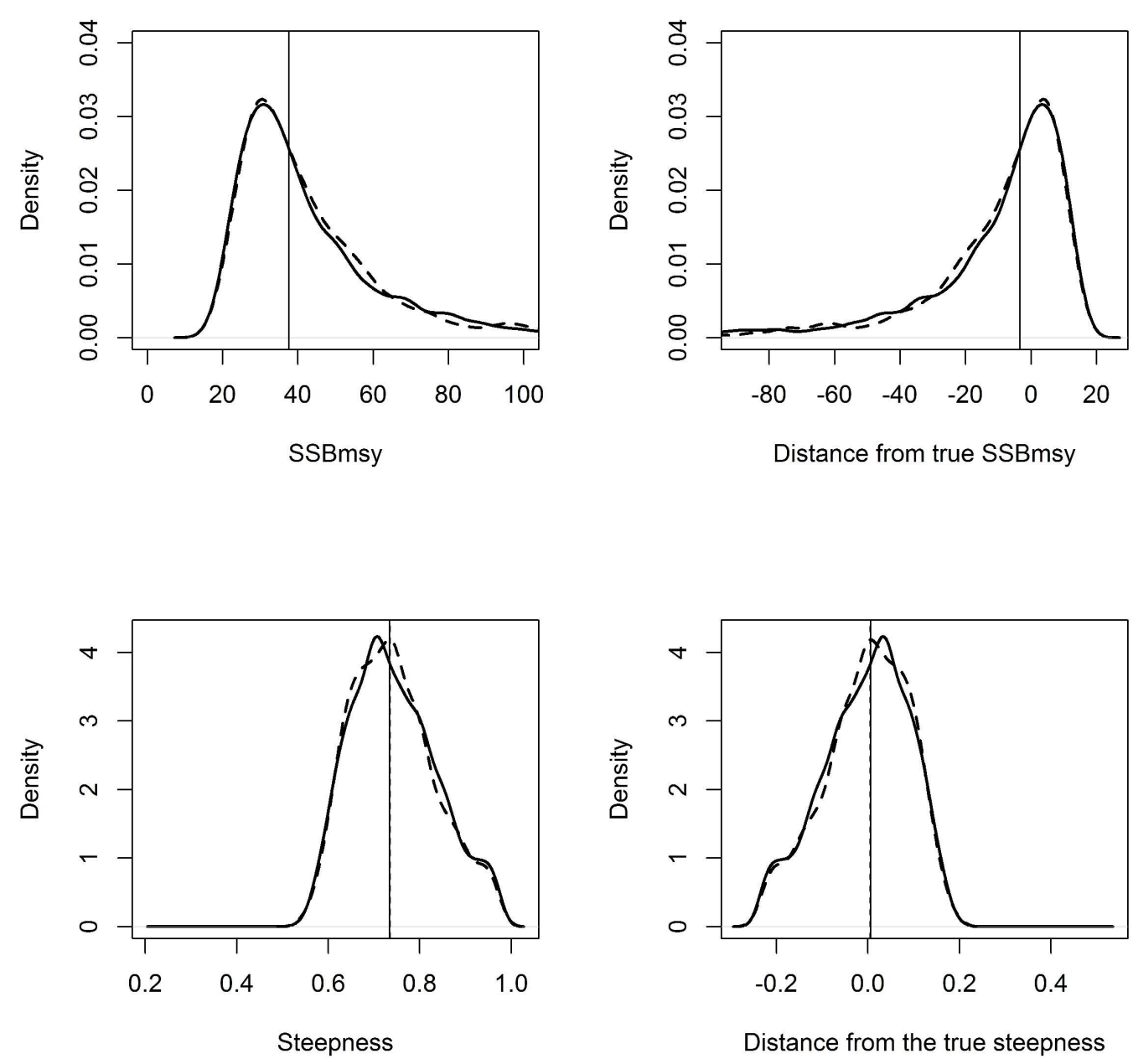

Distance from the true steepness 
Supplementary Material

Figure S10. Recreational CPUE
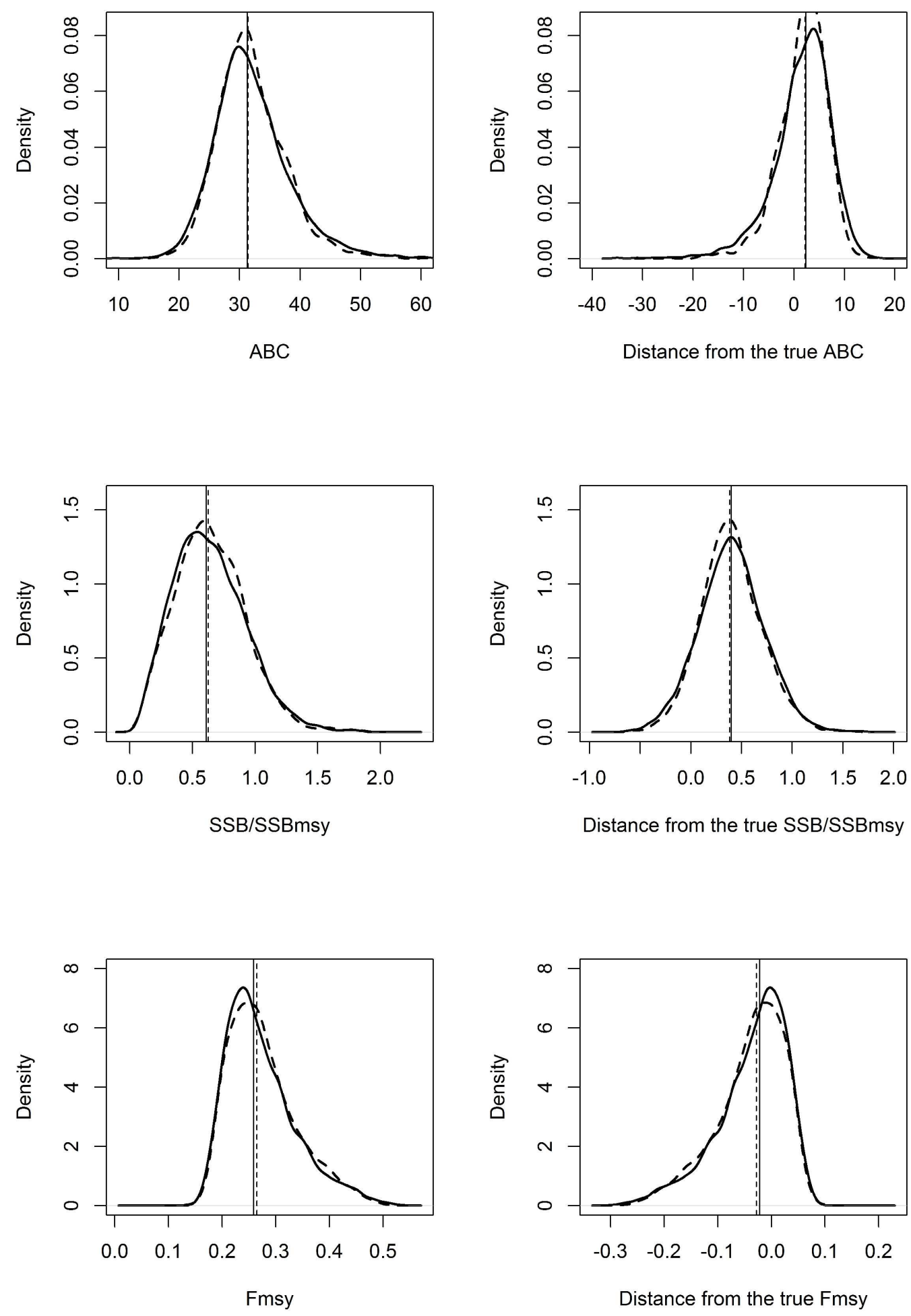
Supplementary Material

Figure S11. Recreational CPUE
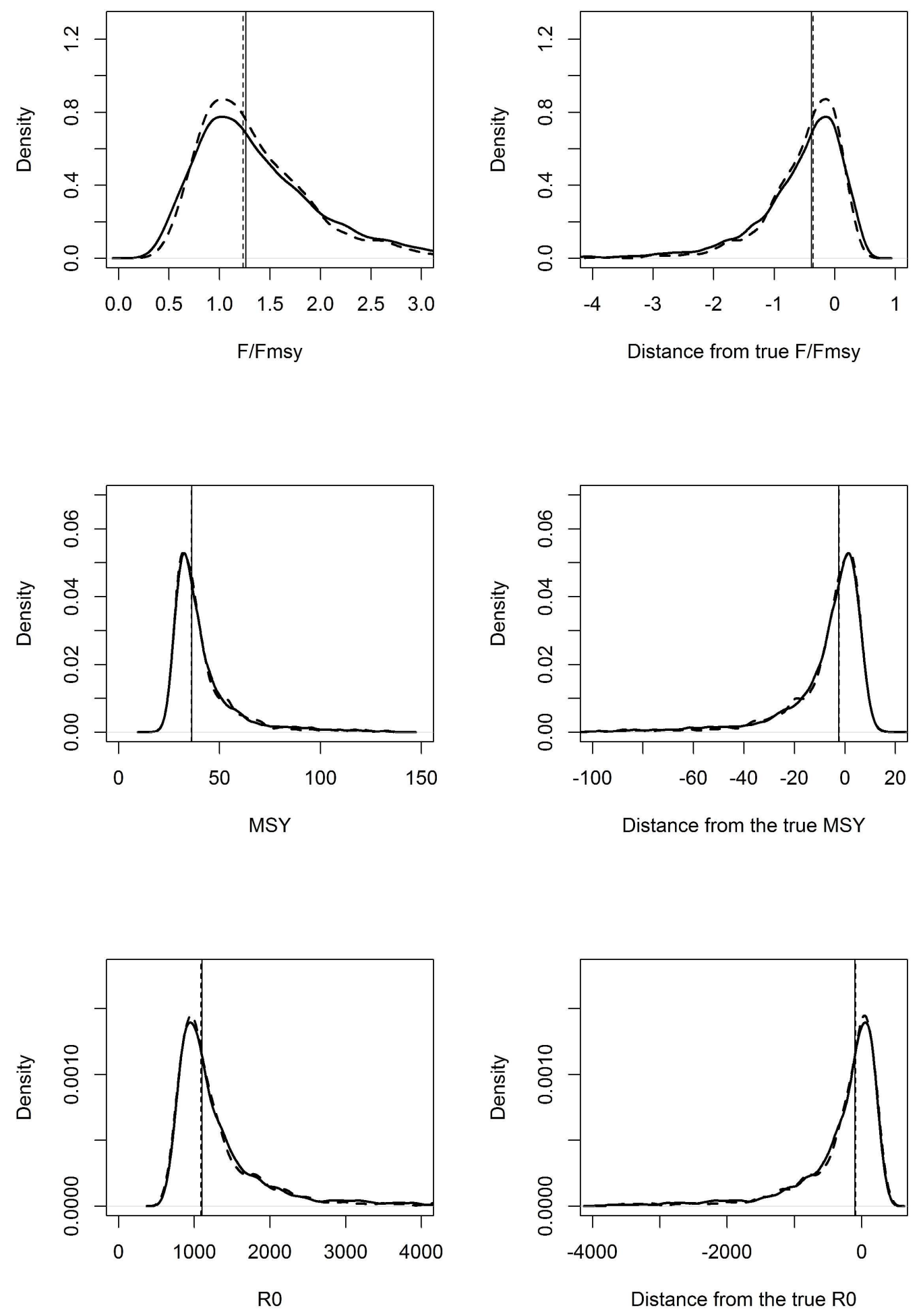
Supplementary Material

Figure S12. Recreational CPUE
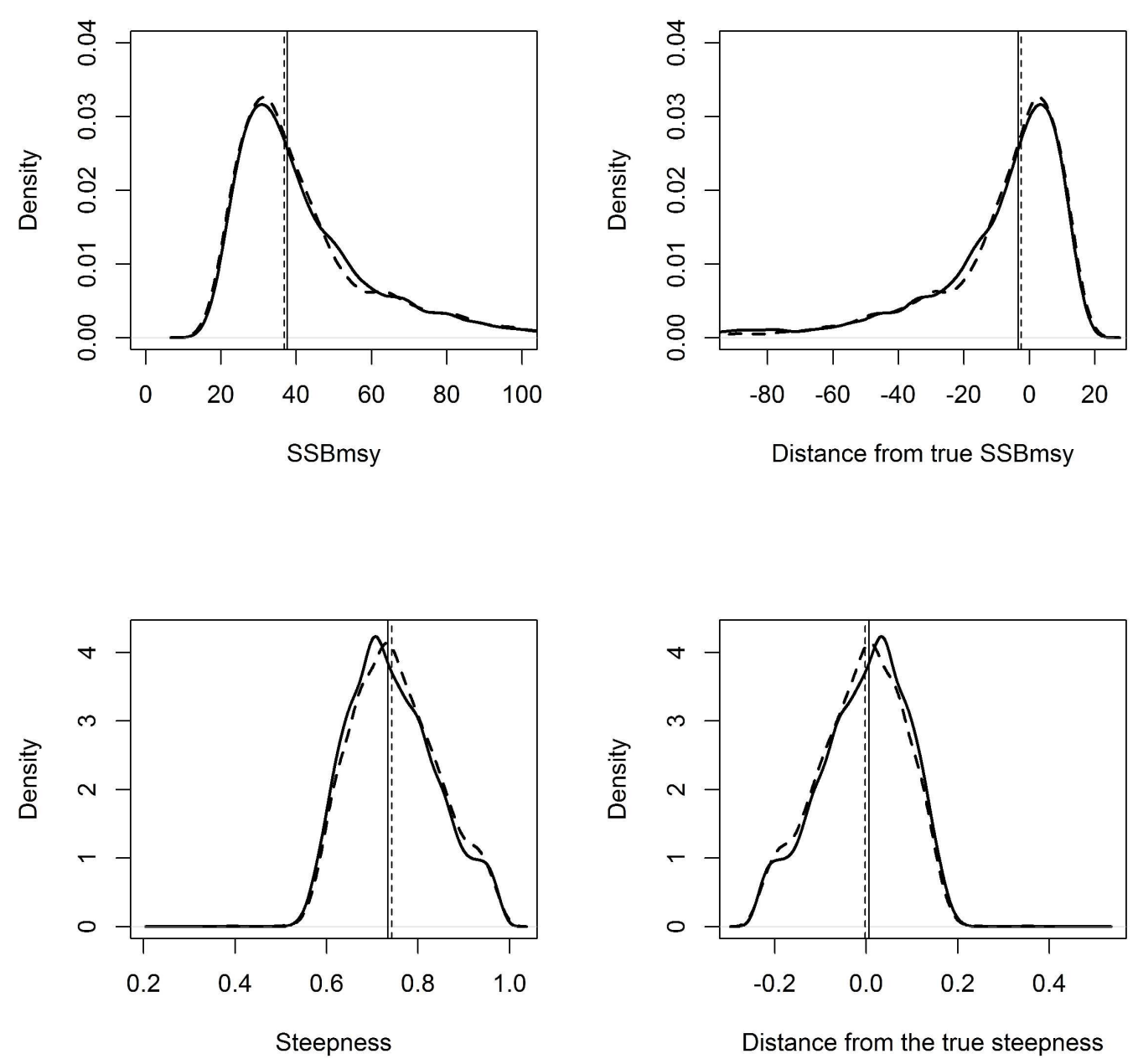
Supplementary Material

Figure S13. Recreational discards
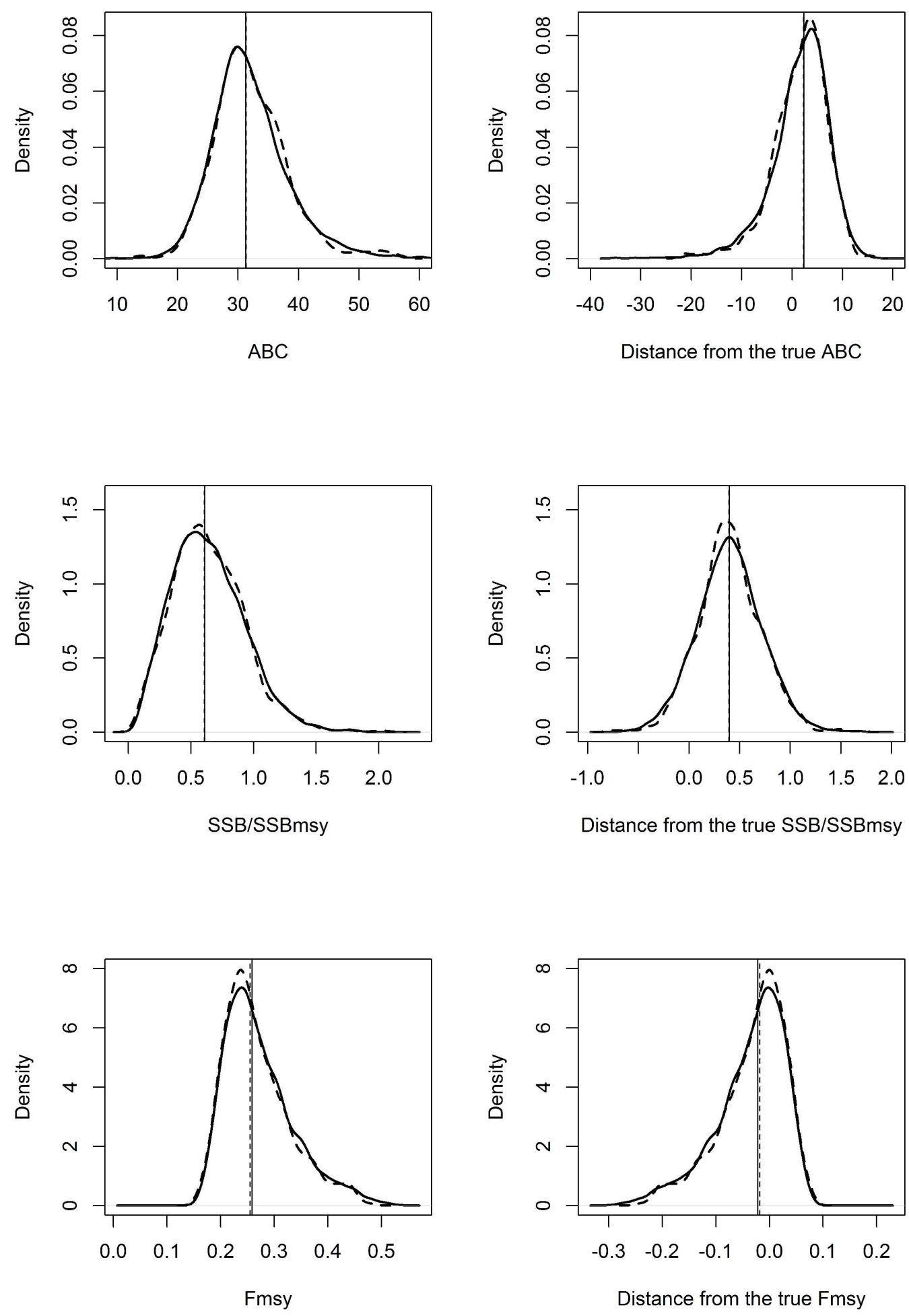
Supplementary Material

Figure S14. Recreational discards
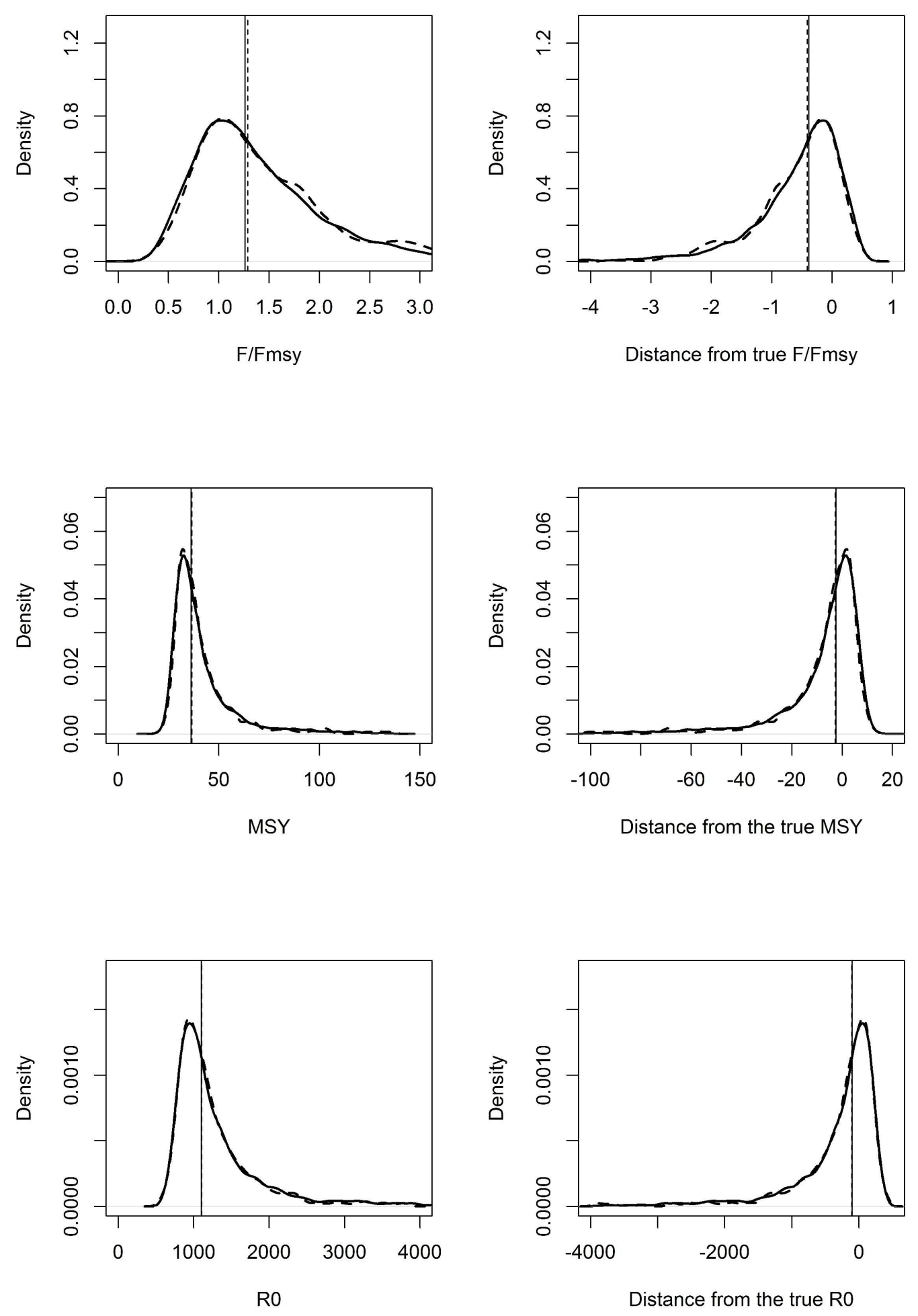
Supplementary Material

Figure S15. Recreational discards
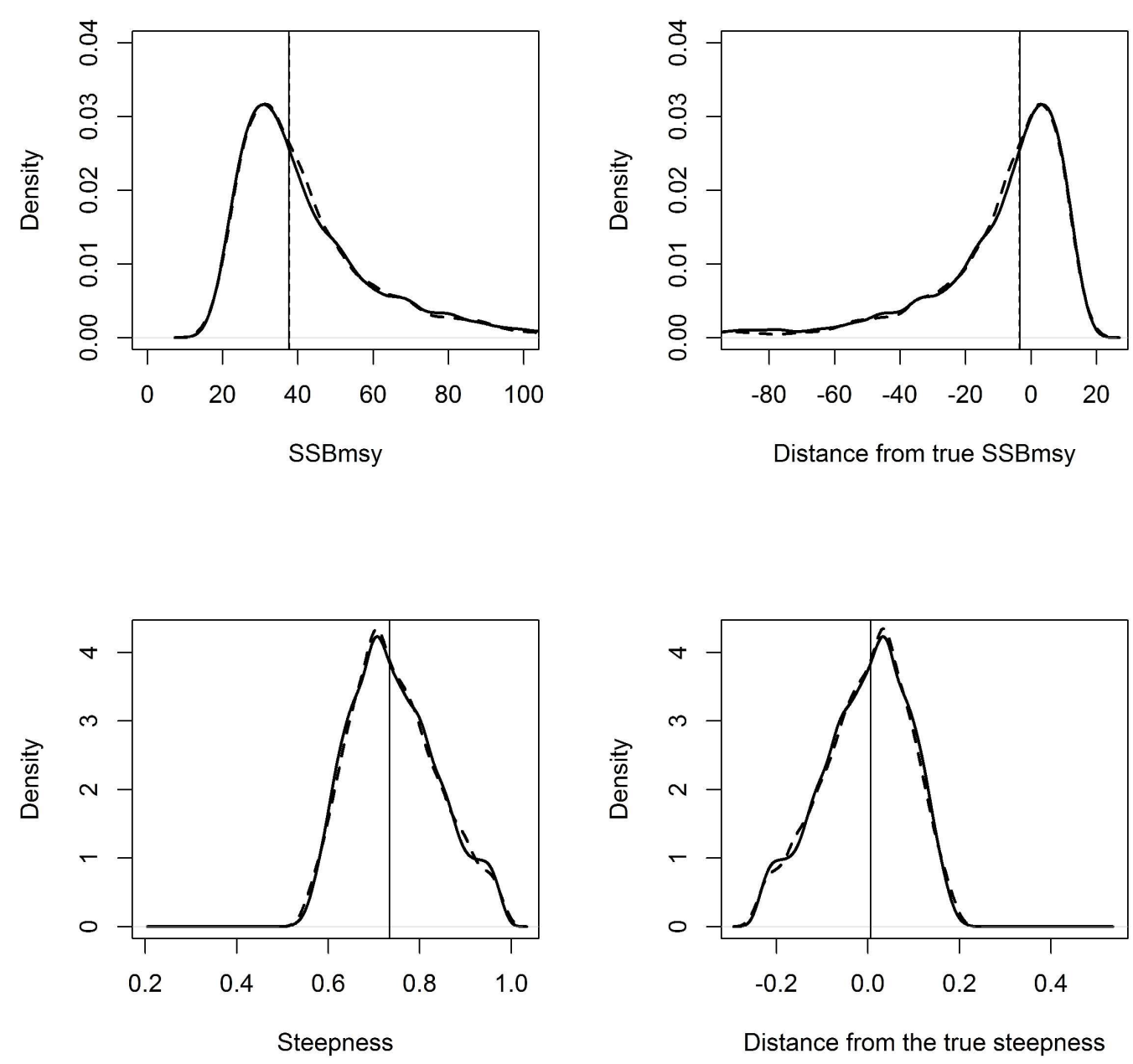

Distance from the true steepness 
Supplementary Material

Figure S16. Recreational landings
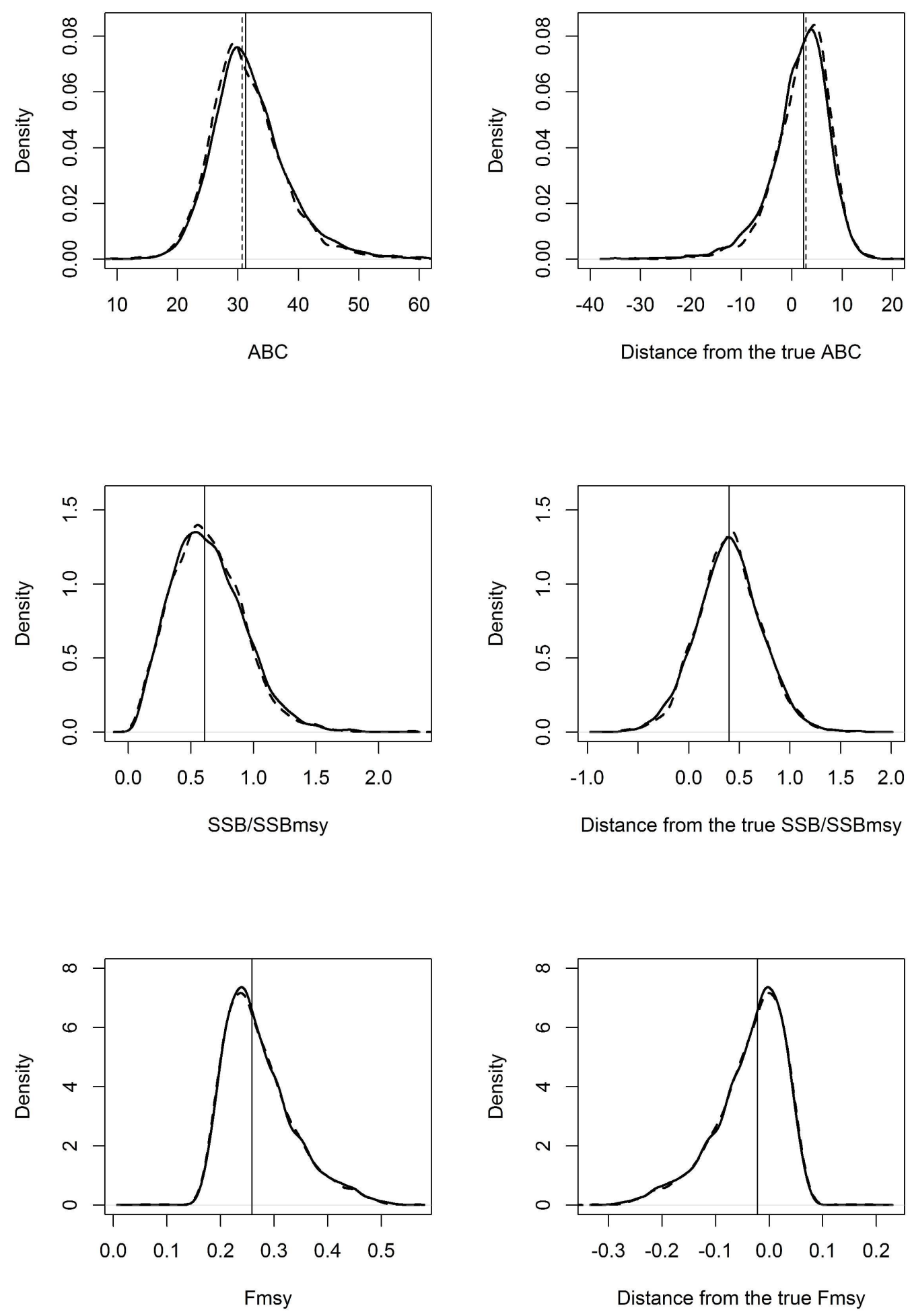
Supplementary Material

Figure S17. Recreational landings
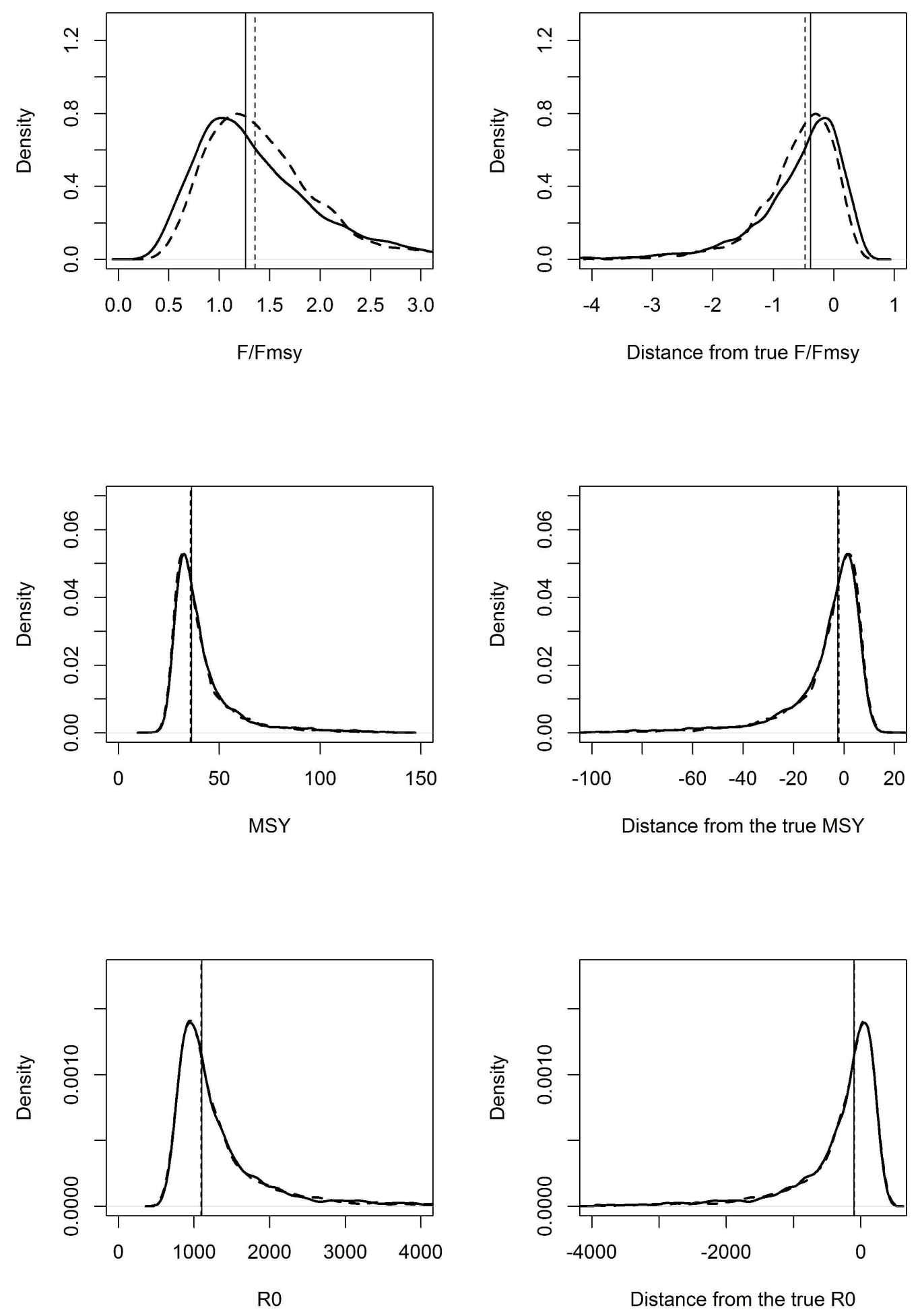
Supplementary Material

Figure S18. Recreational landings
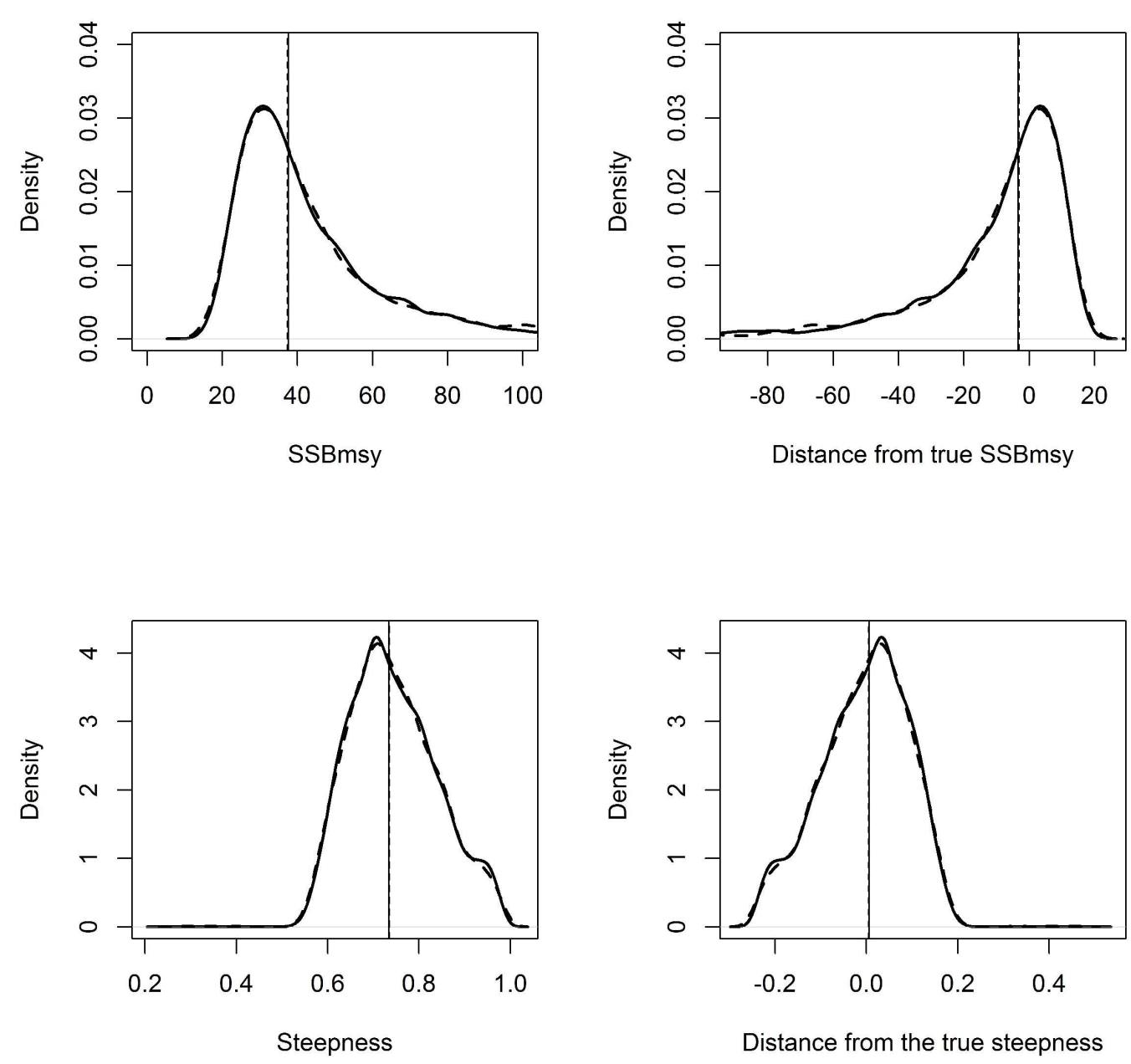

Distance from the true steepness 
Supplementary Material

Figure S19. Survey age compositions (increased sample size)
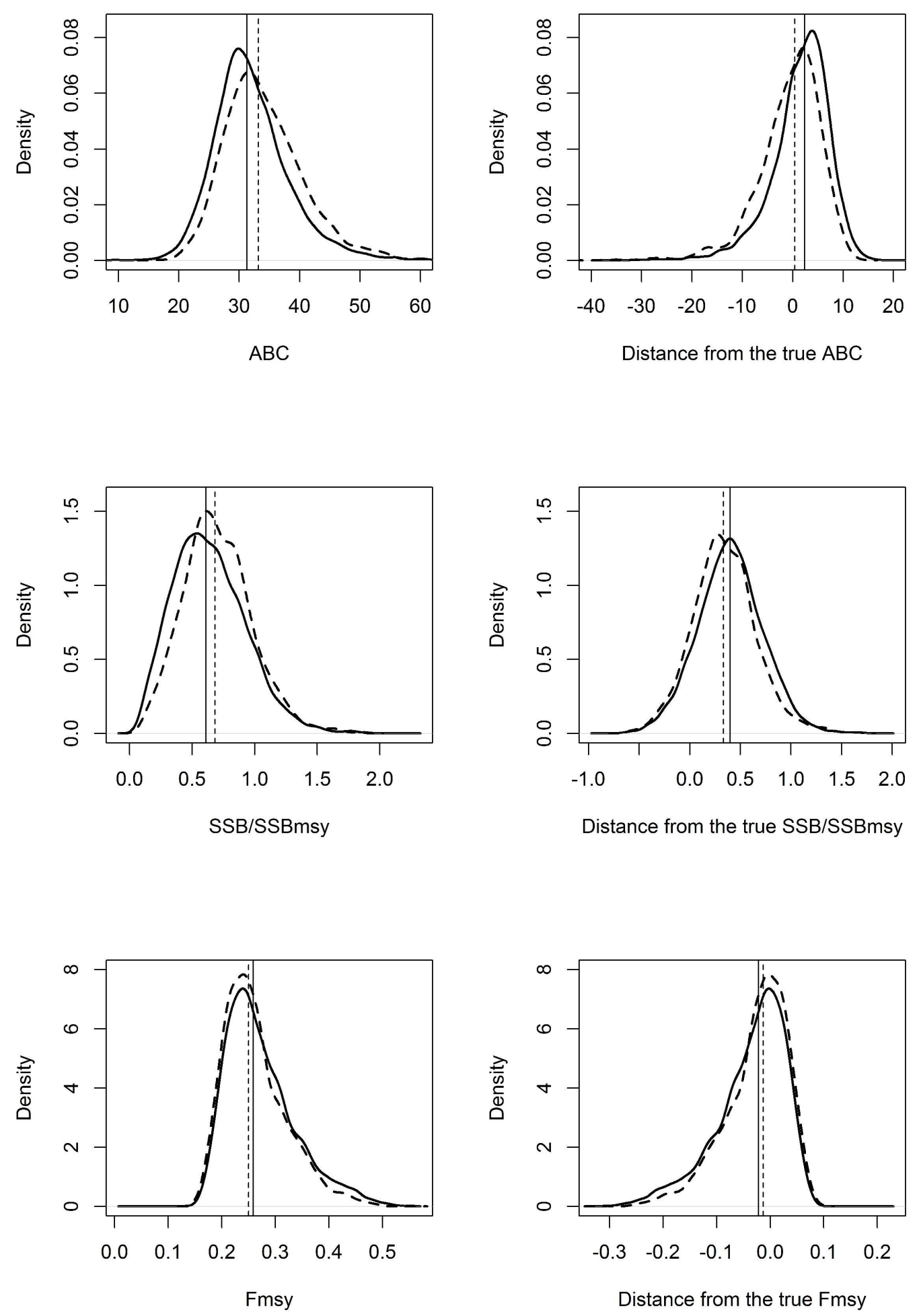
Supplementary Material

Figure S20. Survey age compositions (increased sample size)
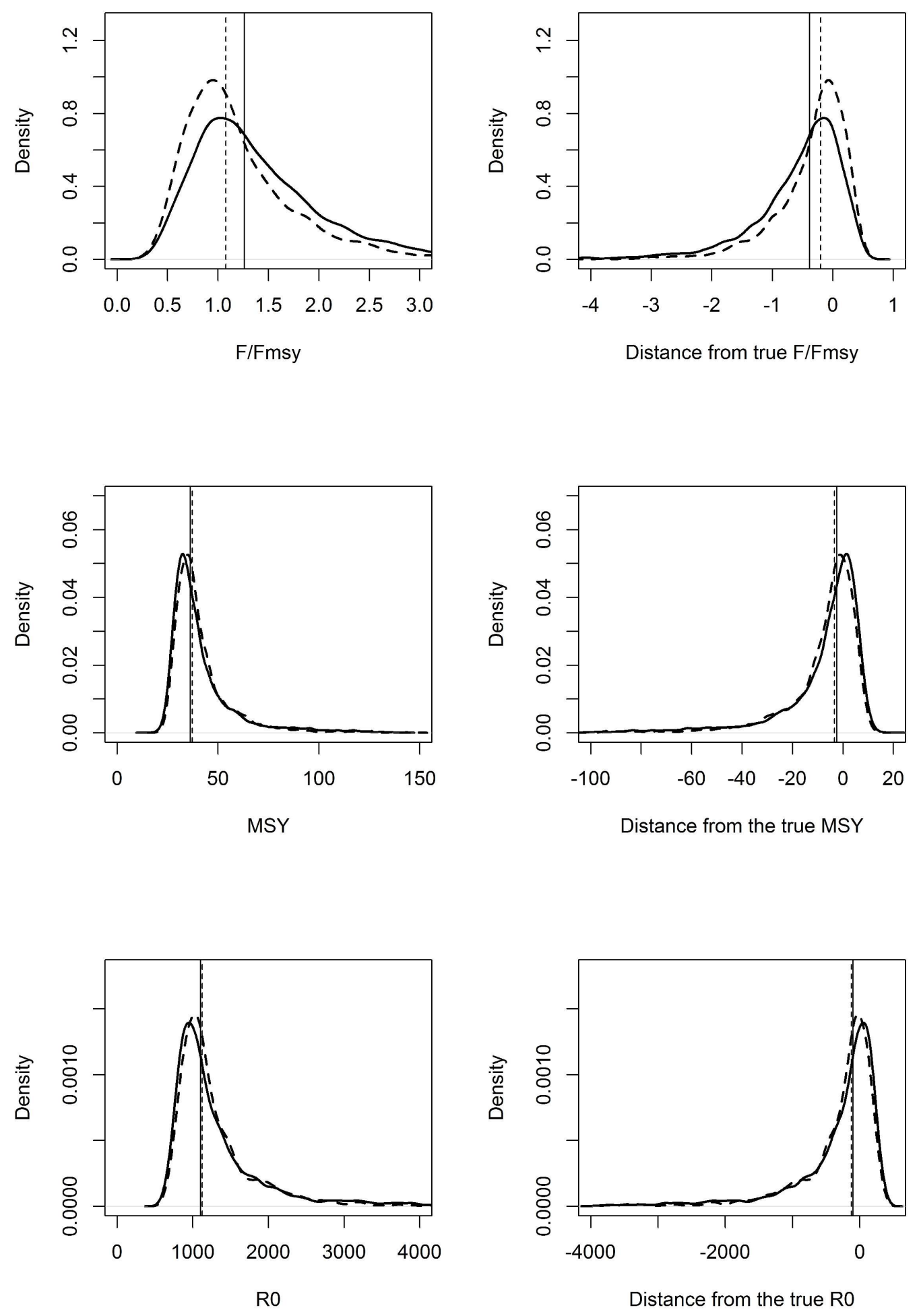
Supplementary Material

Figure S21. Survey age compositions (increased sample size)
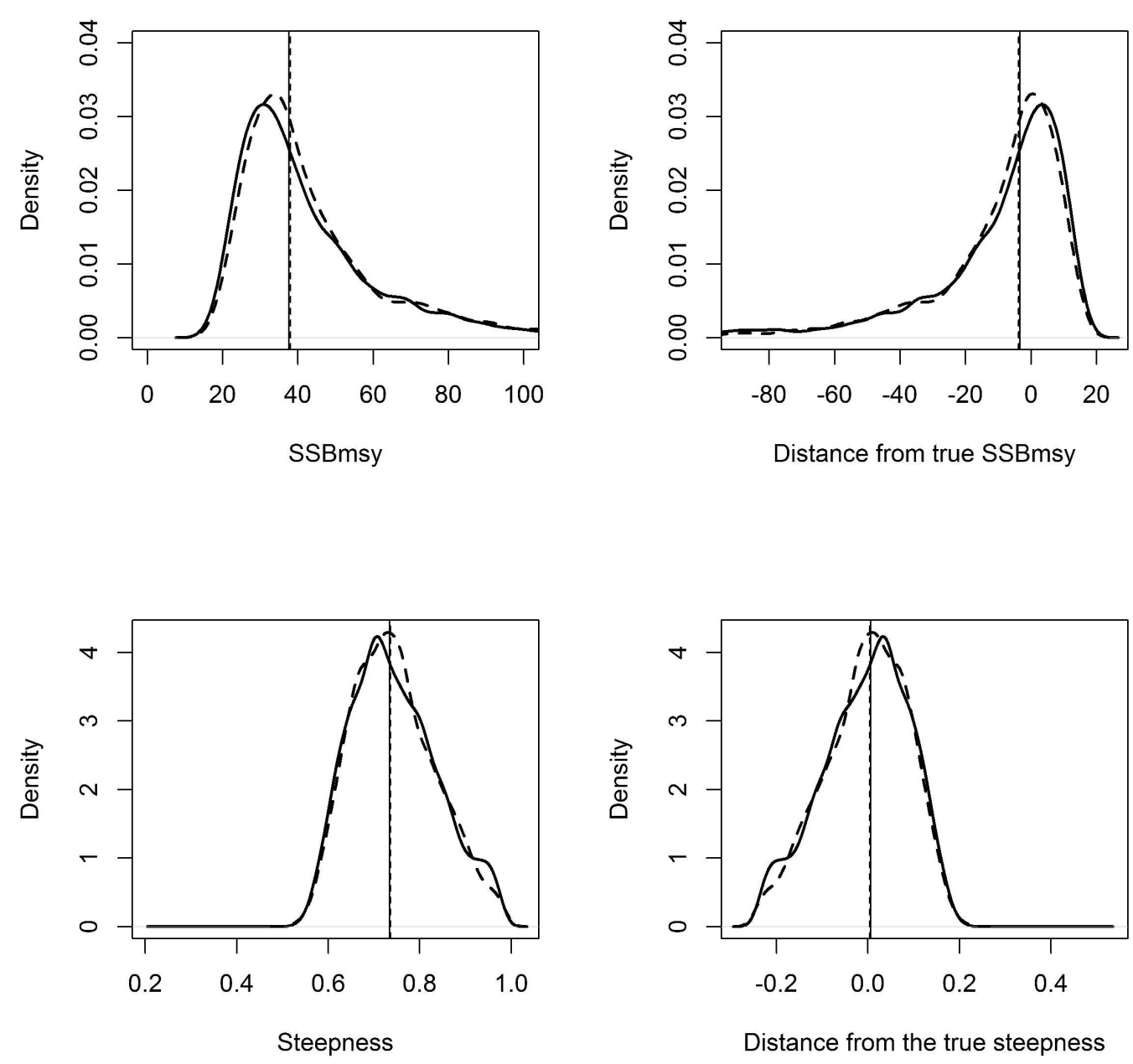

Distance from the true steepness 
Supplementary Material

Figure S22. Survey index
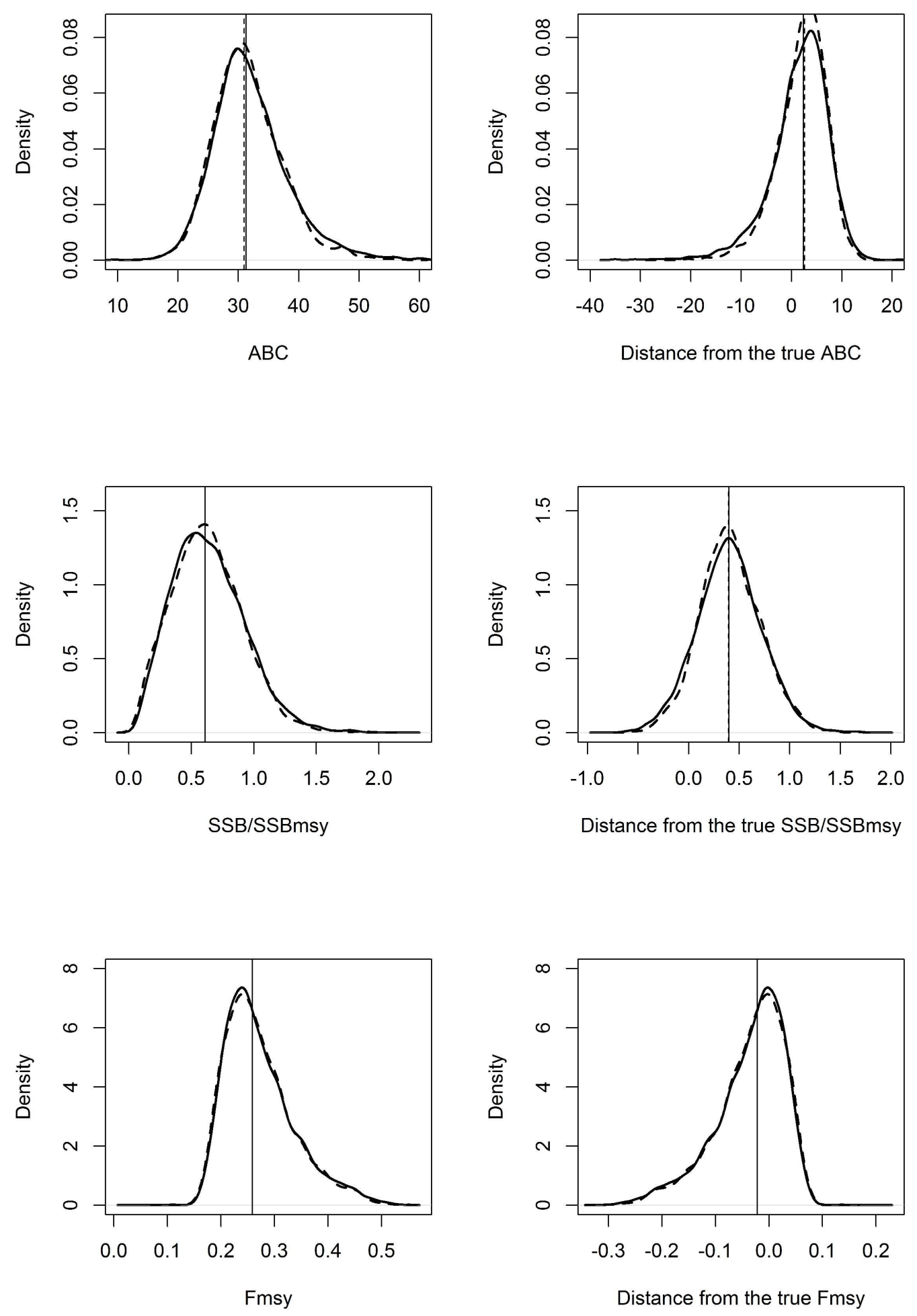
Supplementary Material

Figure S23. Survey index
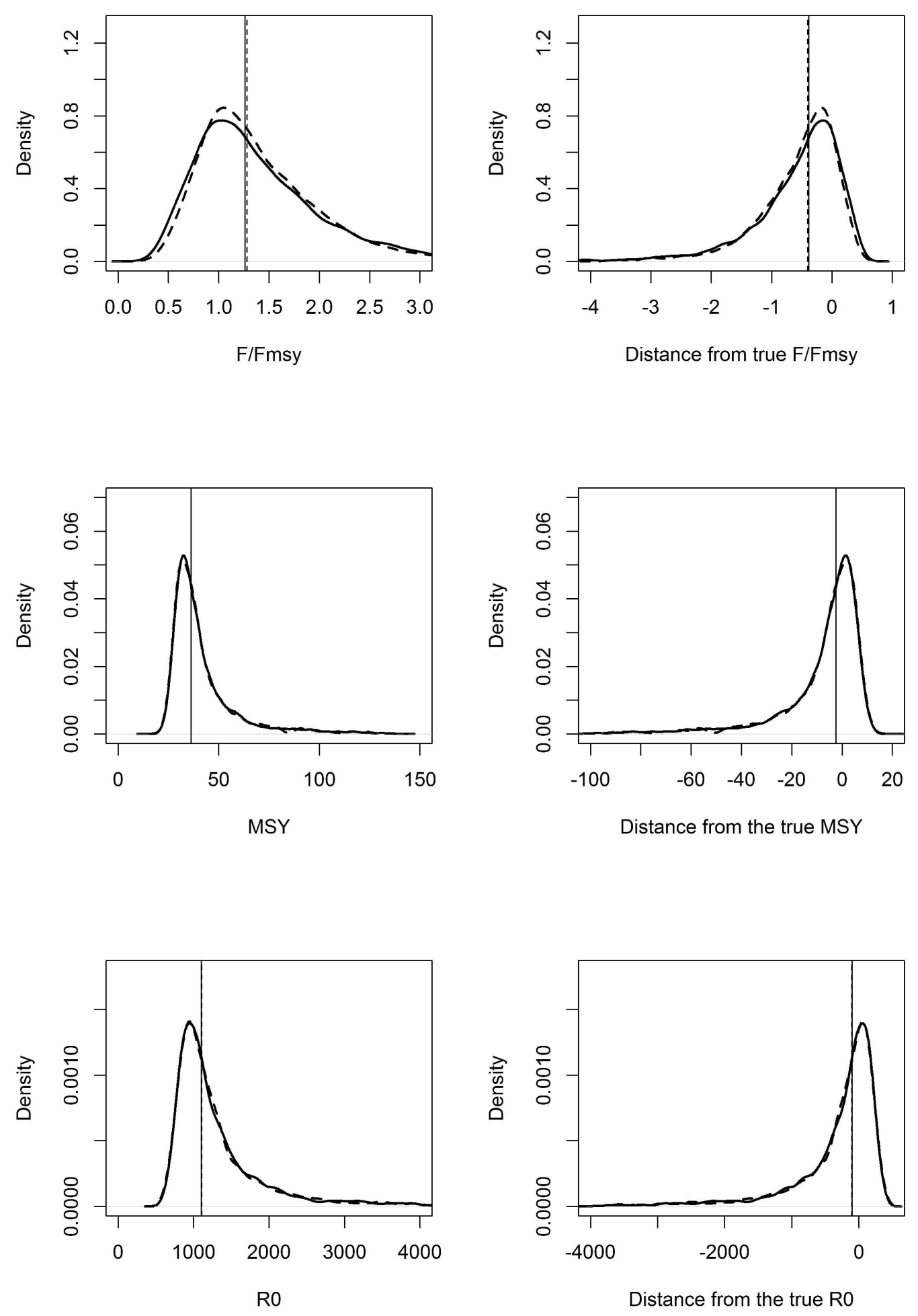
Supplementary Material

Figure S24. Survey index
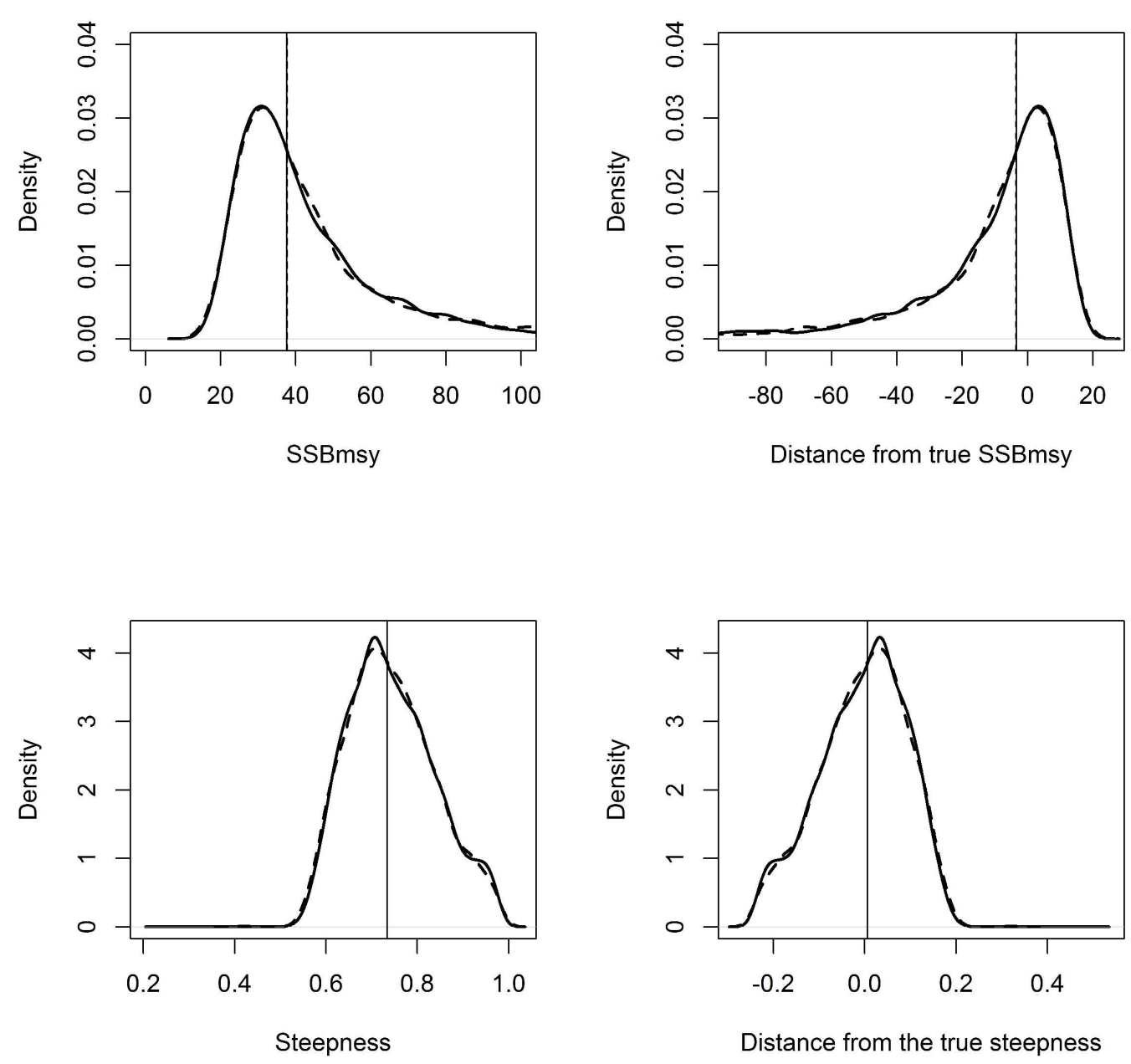

Distance from the true steepness 
Supplementary Material

Figure S25. All commercial components (increased age comp N, decrease discard CV)
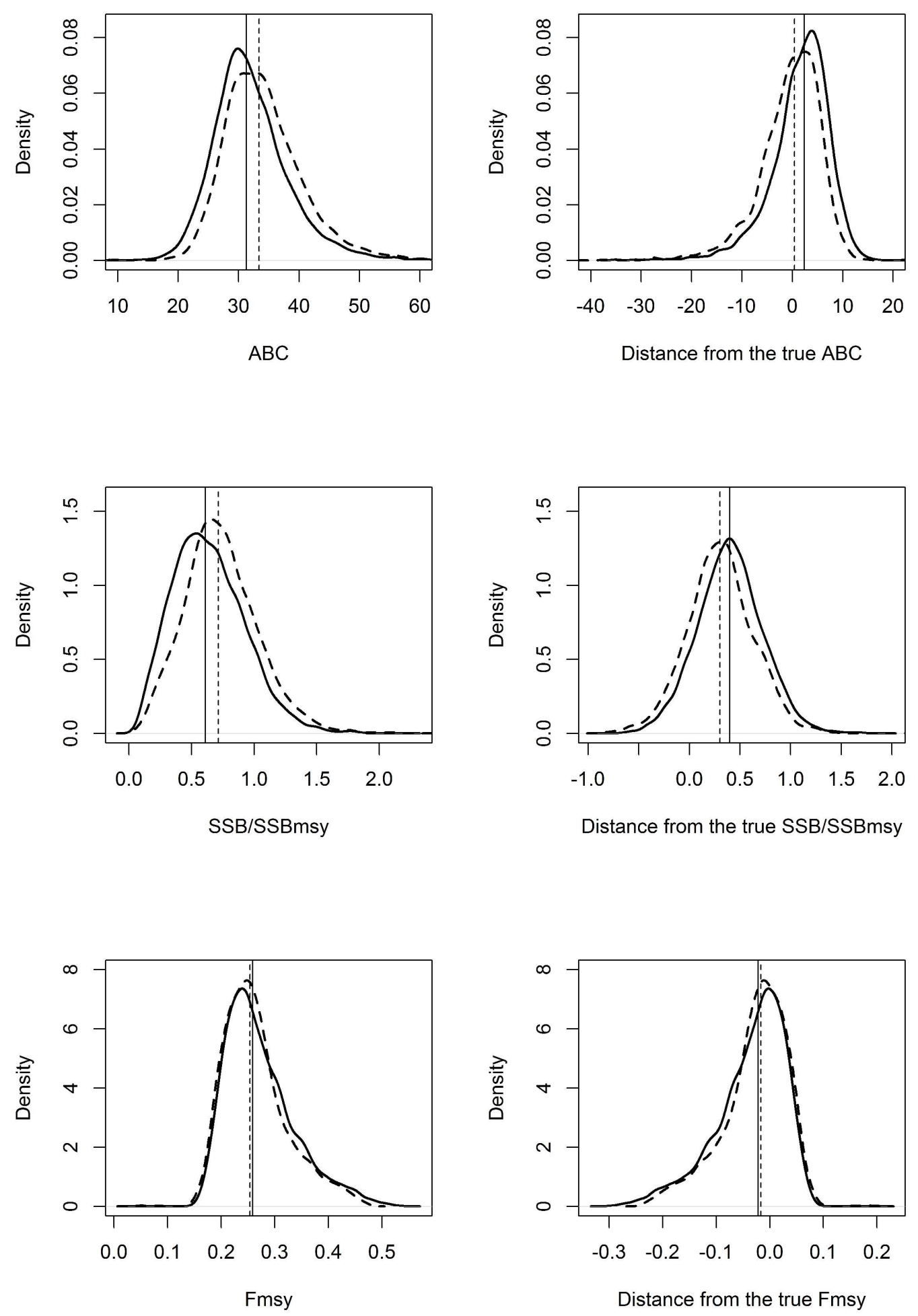
Supplementary Material

Figure S26. All commercial components (increased age comp N, decrease discard CV)
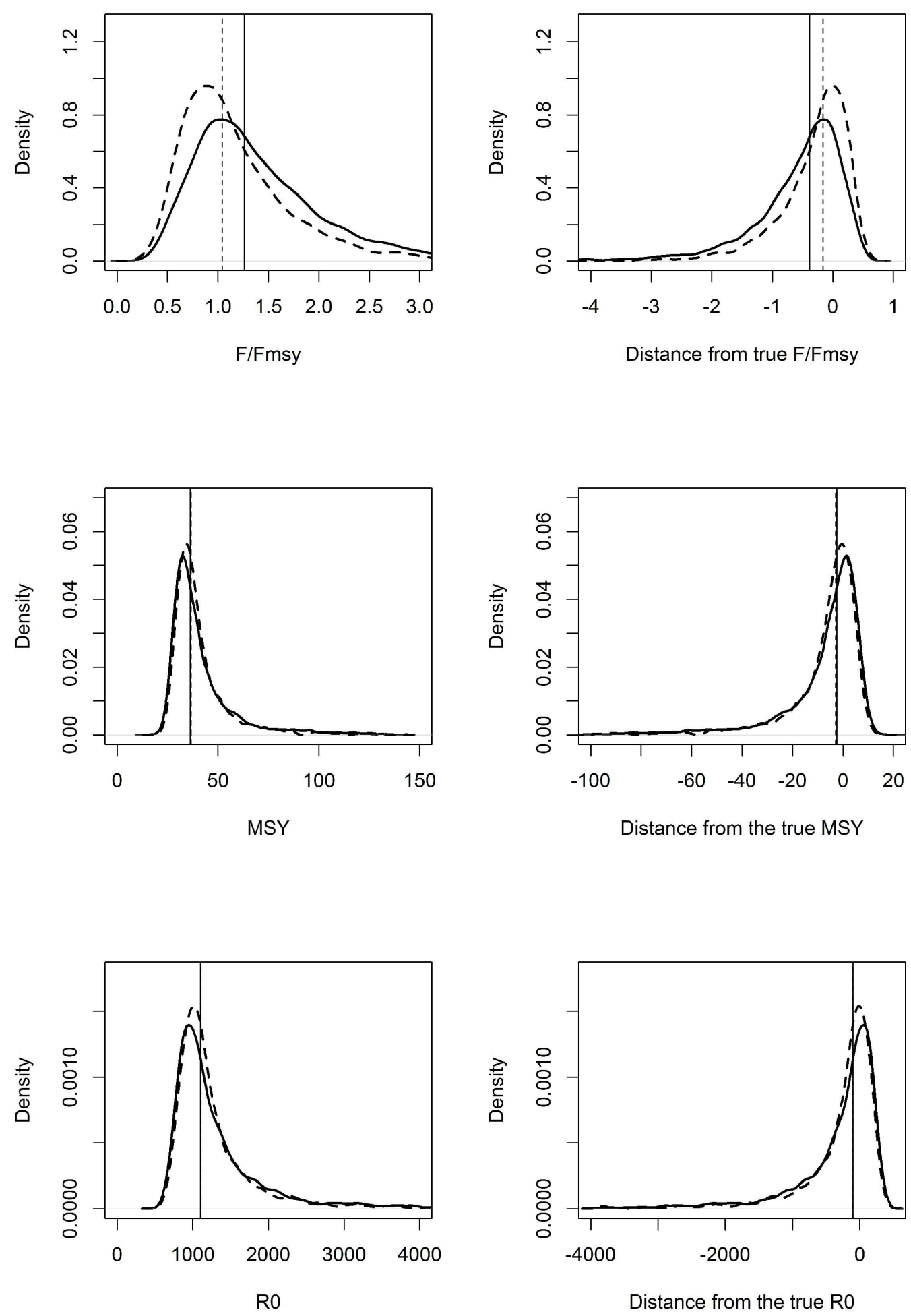
Supplementary Material

Figure S27. All commercial components (increased age comp N, decrease discard CV)
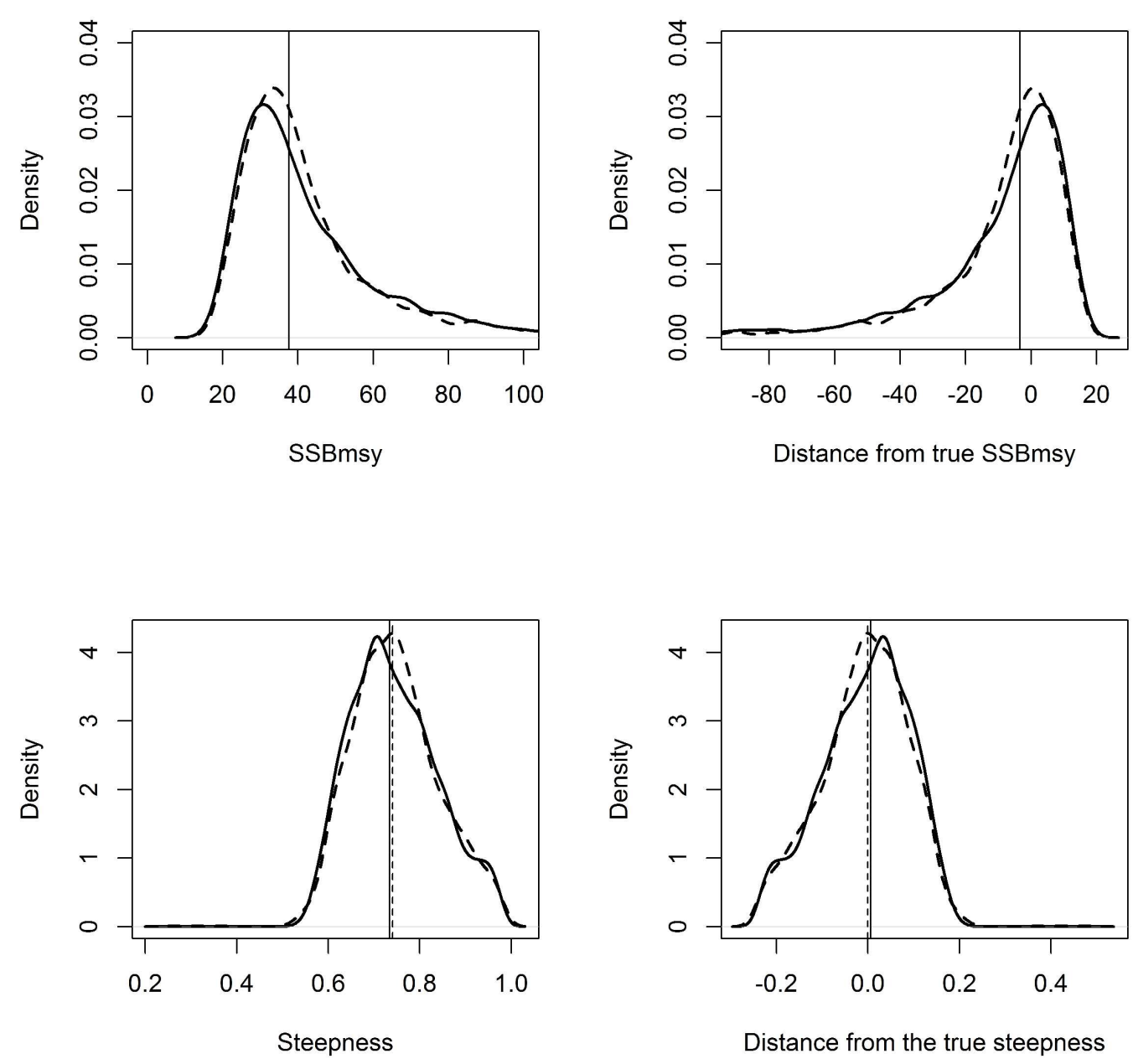

Distance from the true steepness 
Supplementary Material

Figure S28. All recreational components (Increased rec agec sample size, increased discard estimate precision, increased landings precision, decreased CPUE CV)
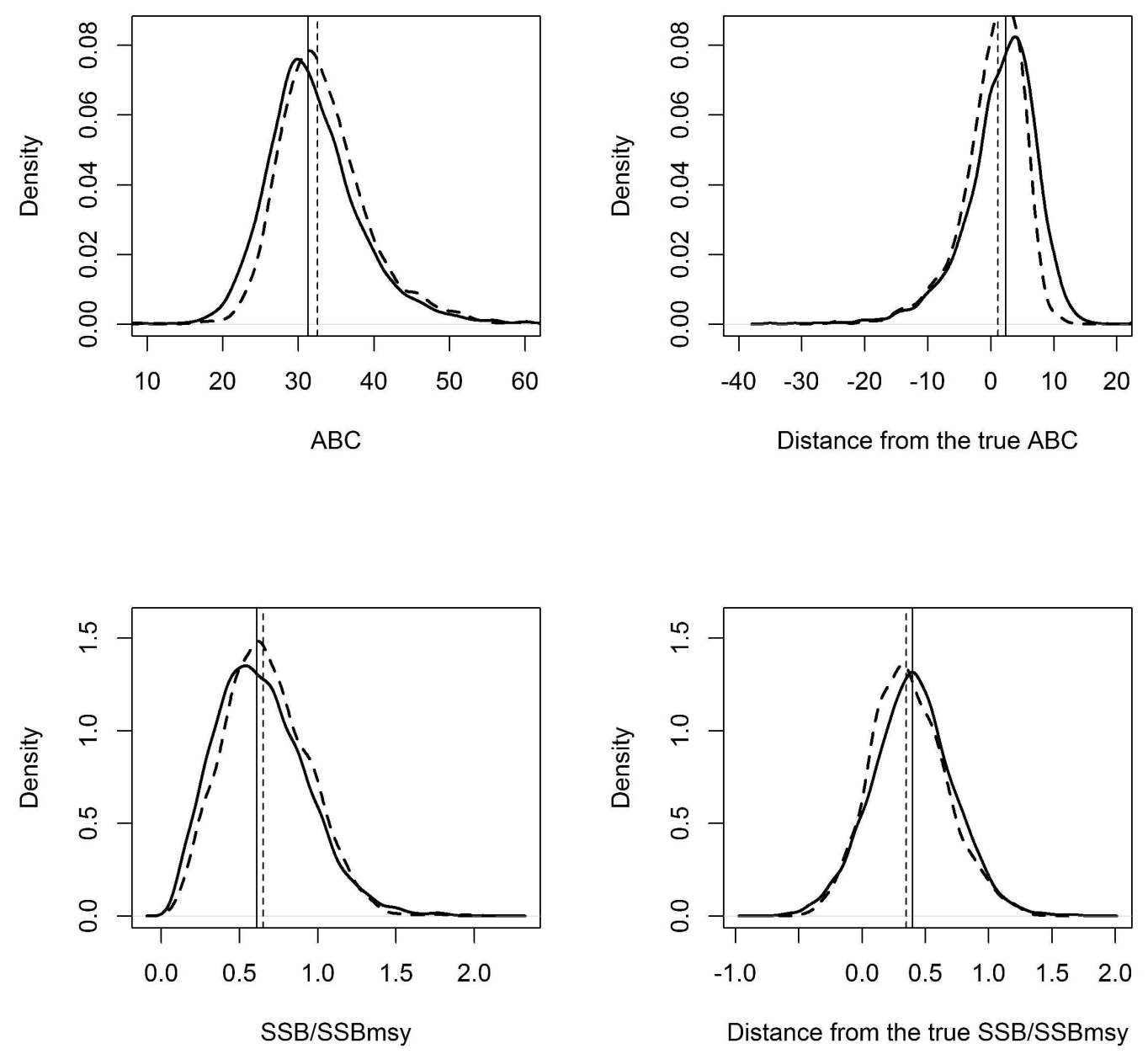
Supplementary Material

Figure S29 All recreational components (Increased rec agec sample size, increased discard estimate precision, increased landings precision, decreased CPUE CV)
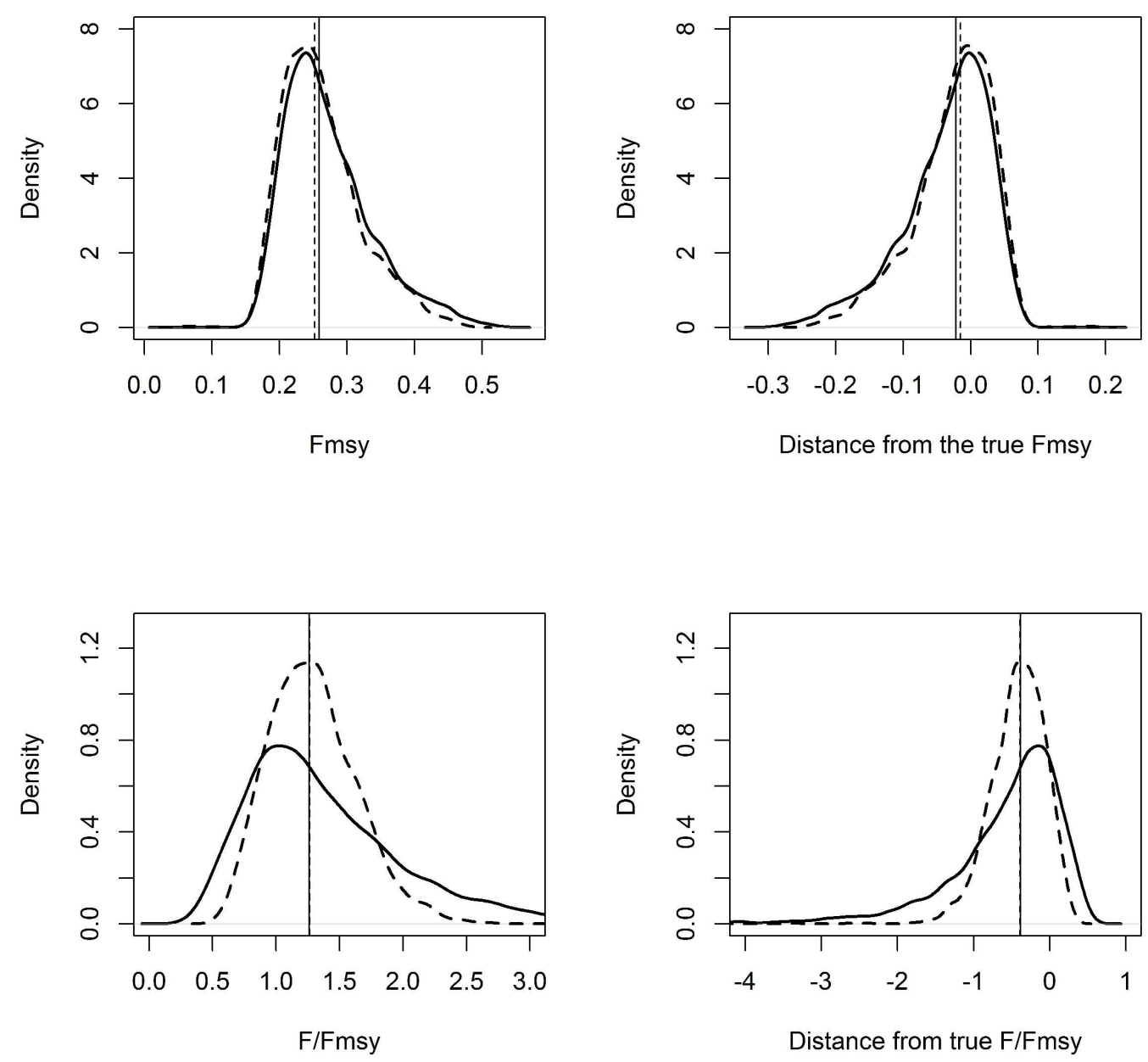
Supplementary Material

Figure S30. All recreational components (Increased rec agec sample size, increased discard estimate precision, increased landings precision, decreased CPUE CV)
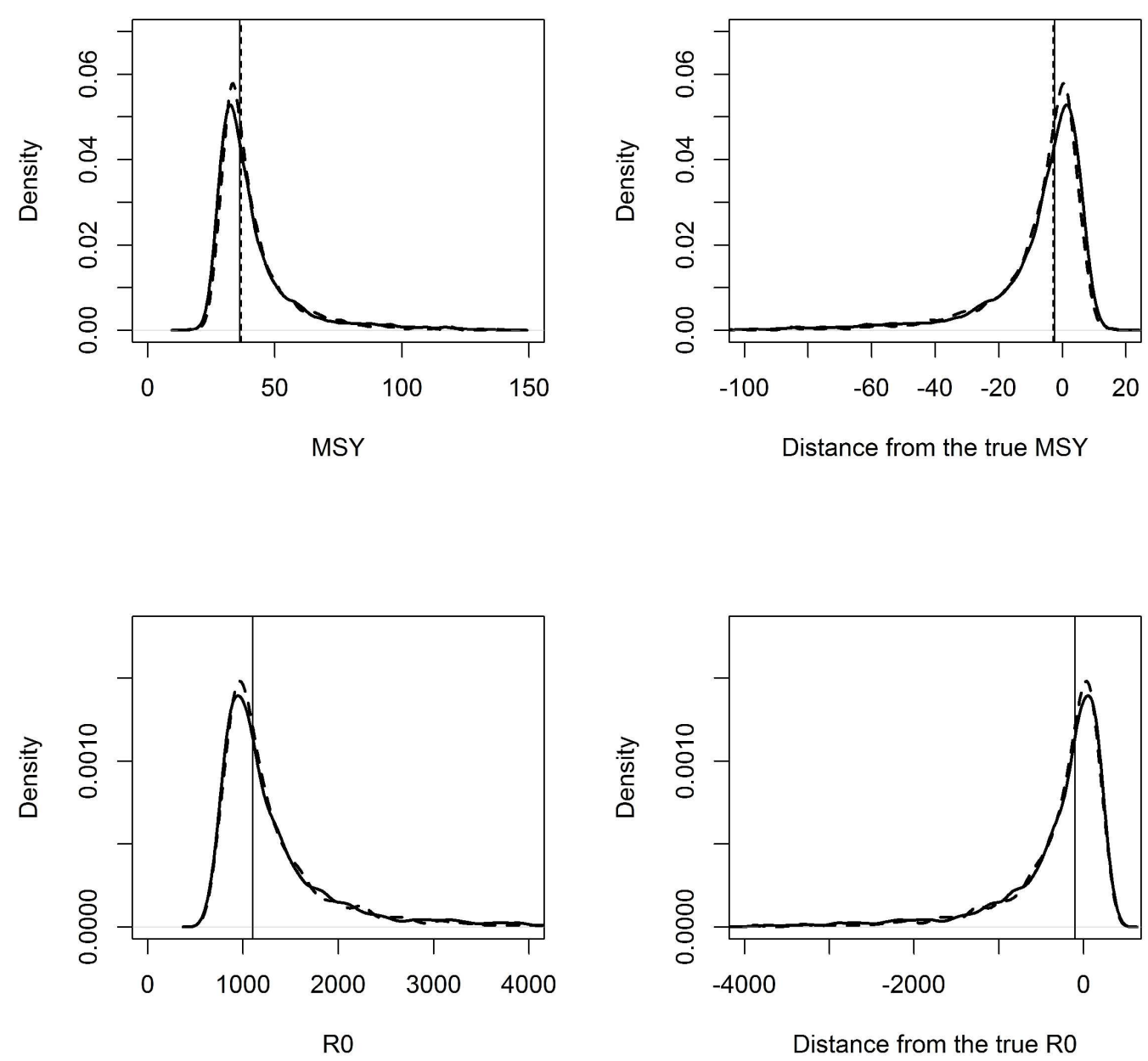
Supplementary Material

Figure S31. All recreational components (Increased rec agec sample size, increased discard estimate precision, increased landings precision, decreased CPUE CV)
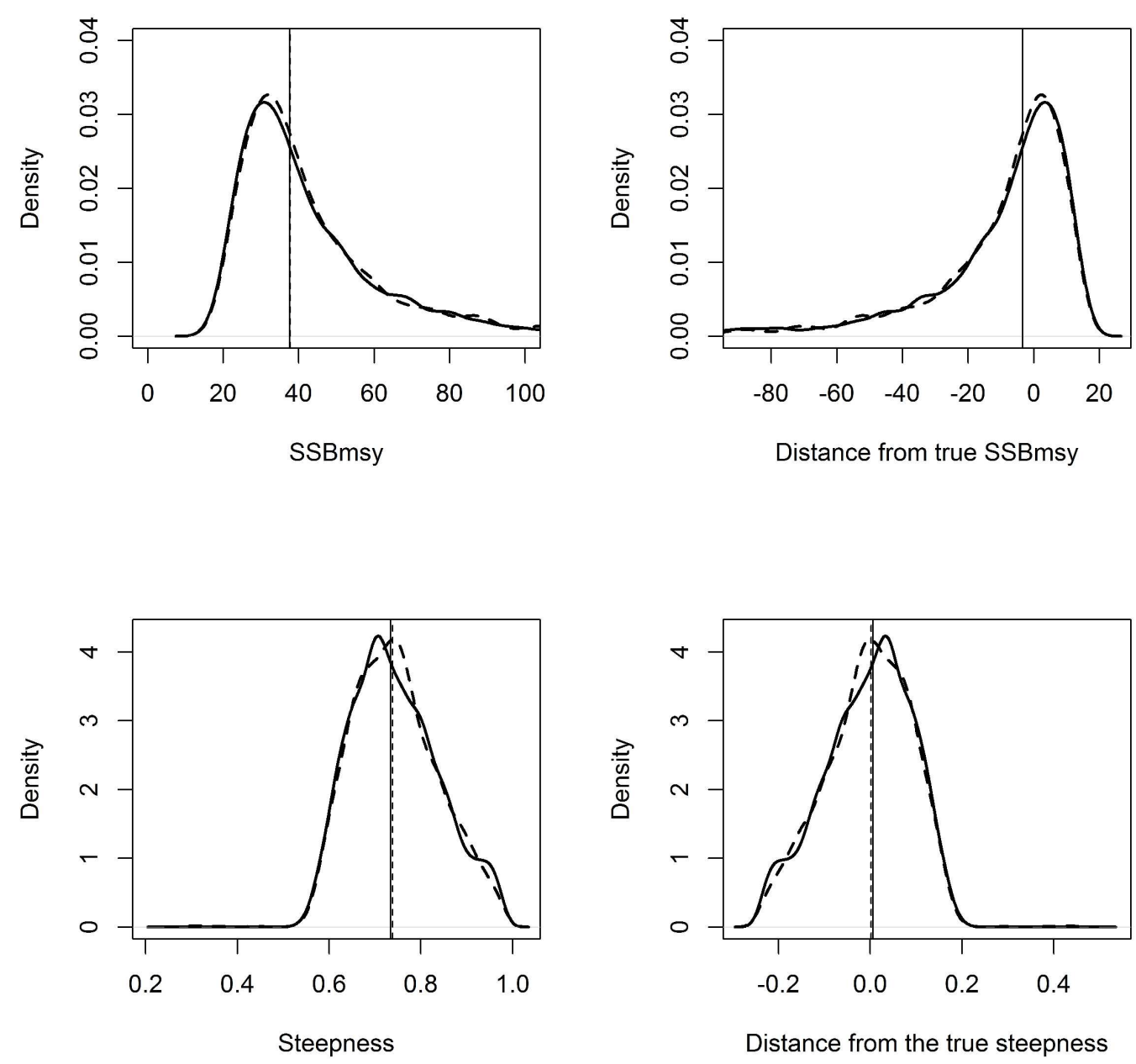

Distance from the true steepness 
Supplementary Material

Figure S32. All indices
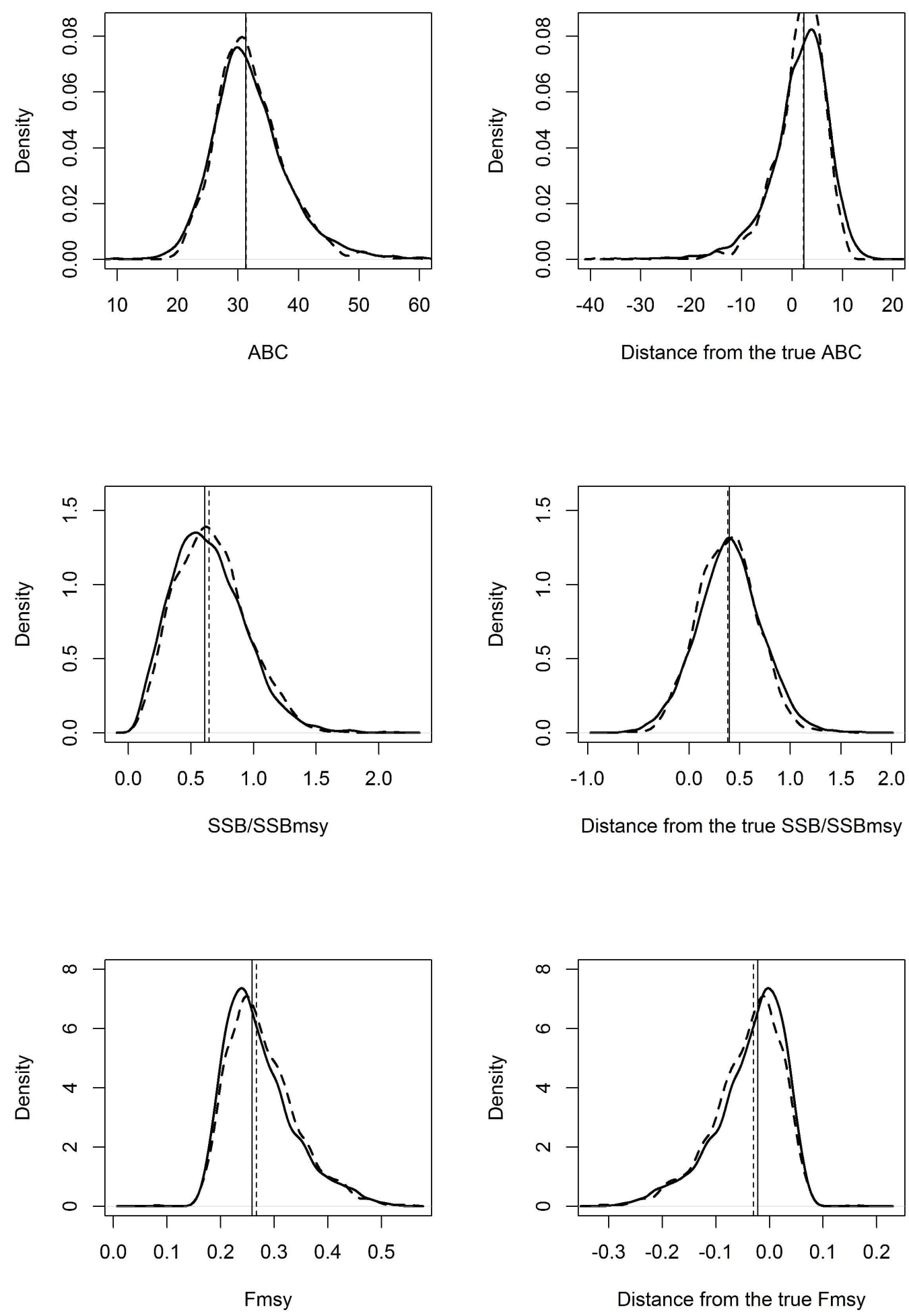
Supplementary Material

Figure S33. All indices
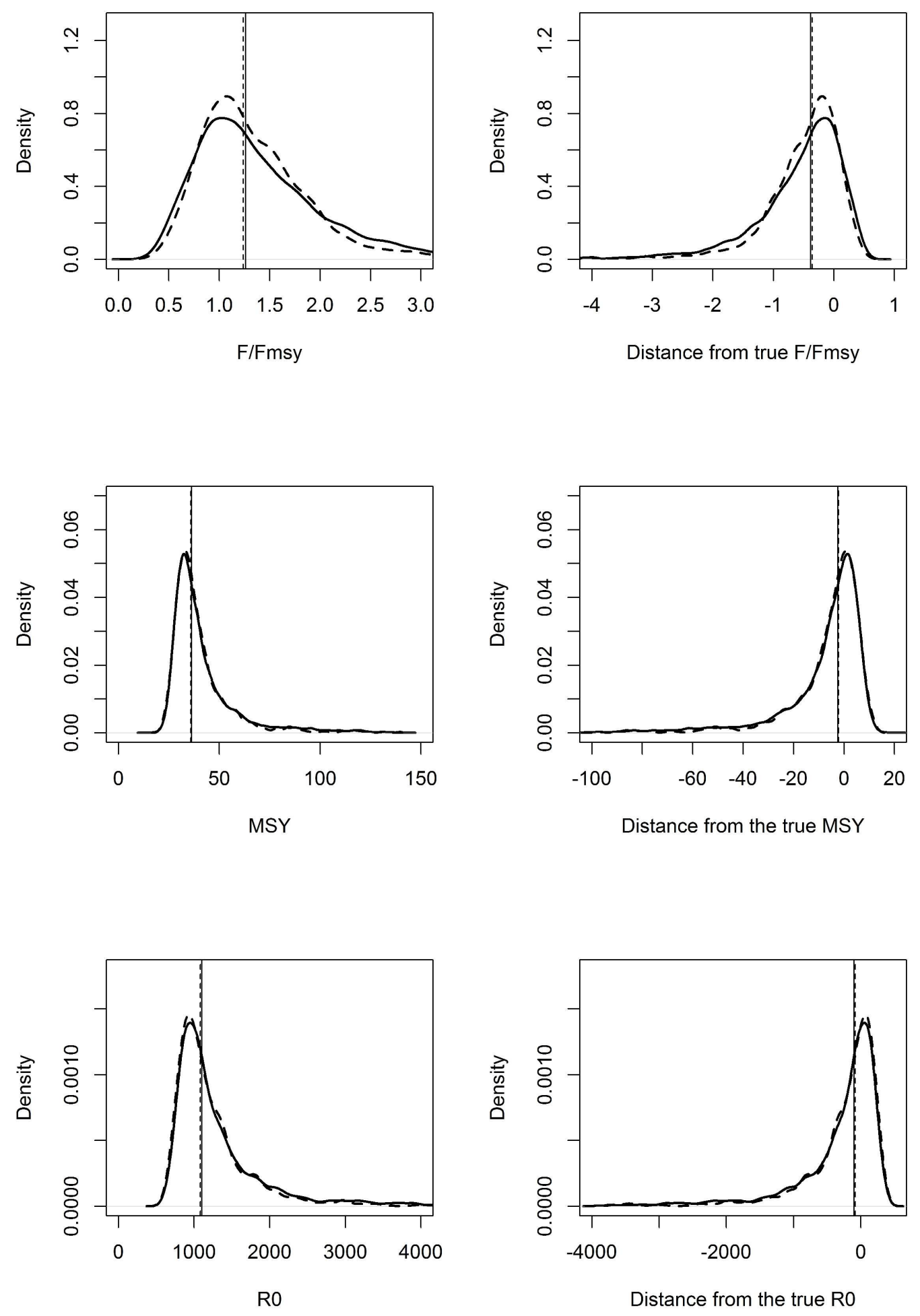
Supplementary Material

Figure S34. All indices
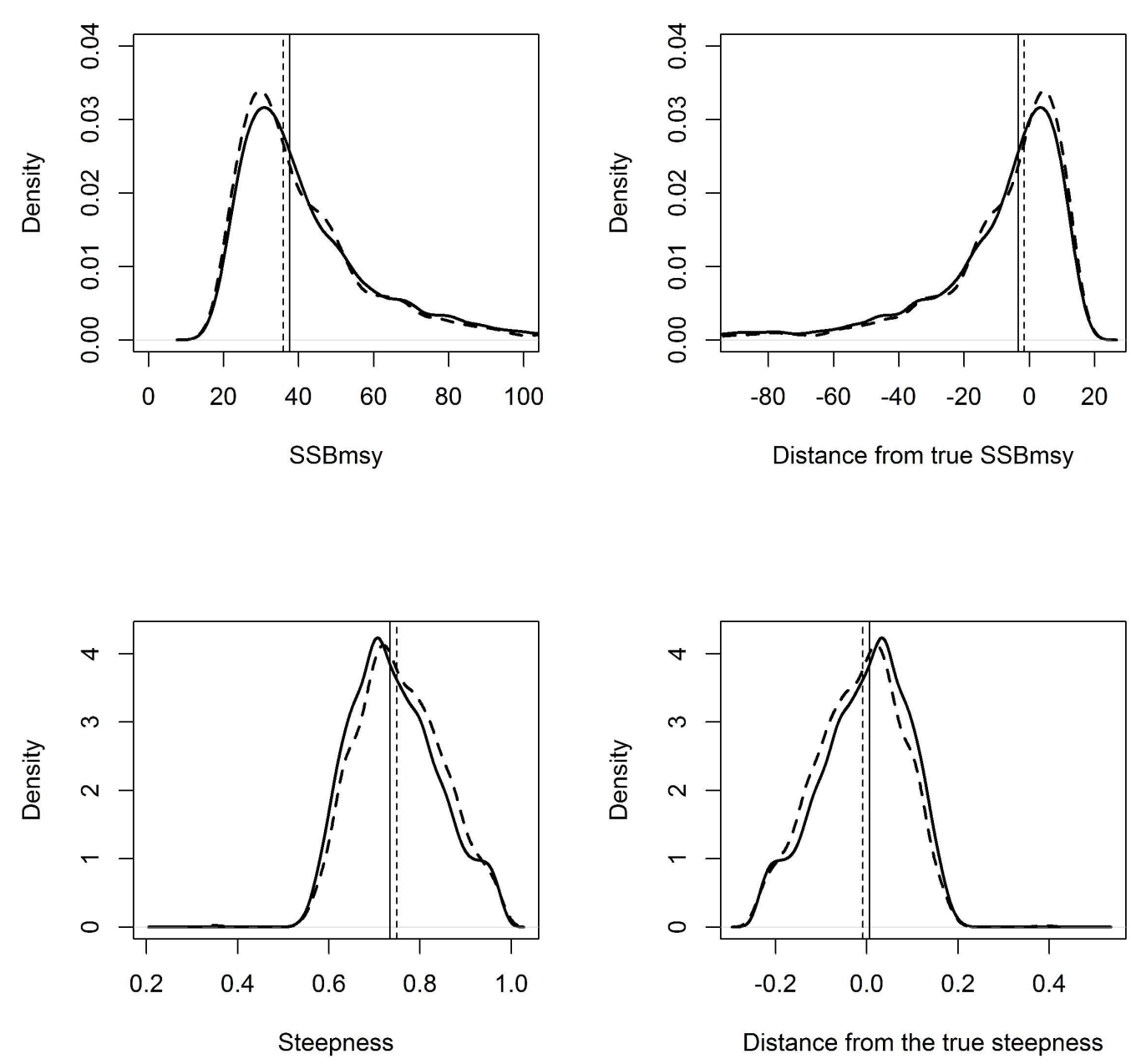

Distance from the true steepness 
Supplementary Material

Figure S35. All survey components (increased sample size)
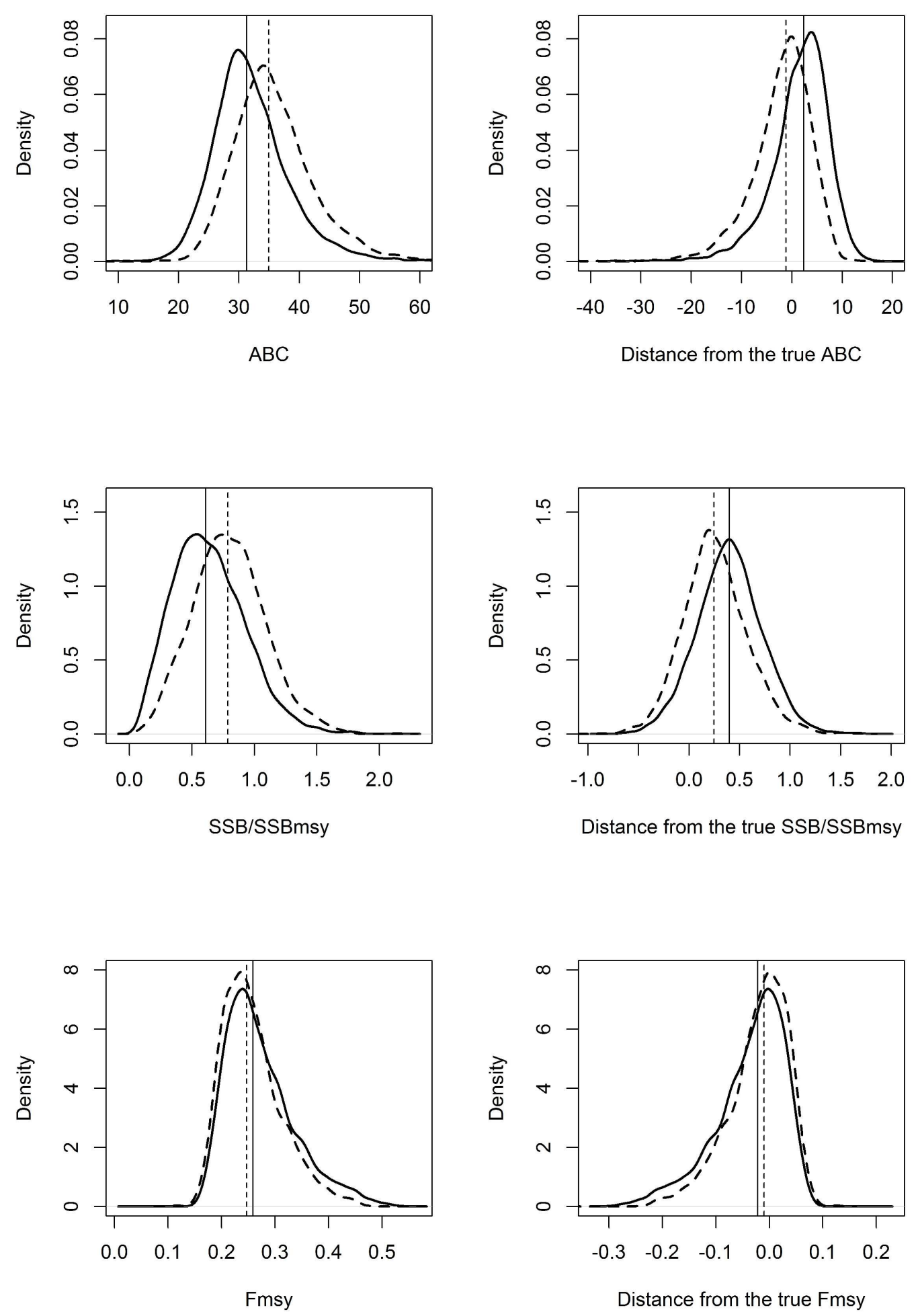
Supplementary Material

Figure S36. All survey components (increased sample size)
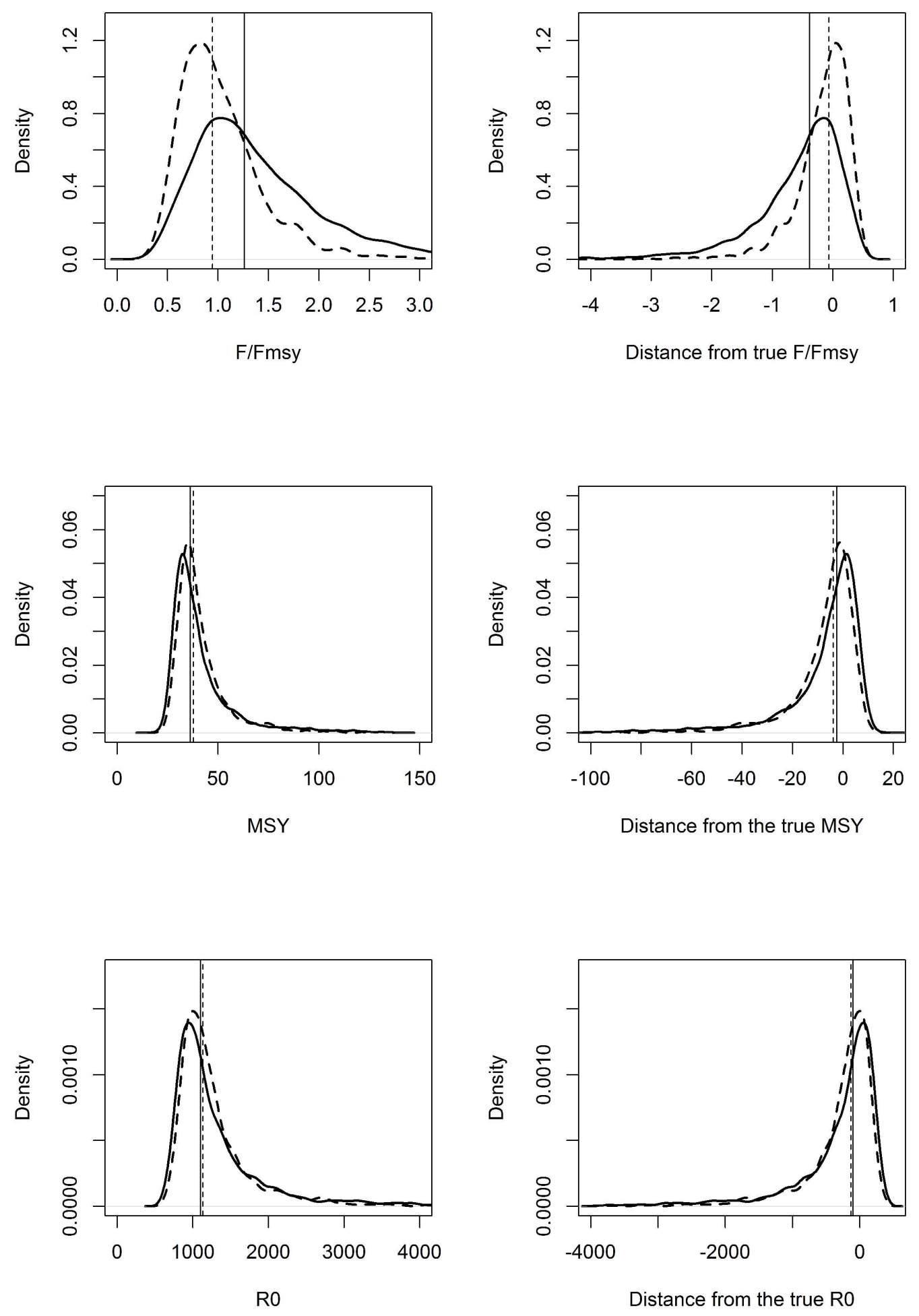
Supplementary Material

Figure S37. All survey components (increased sample size)
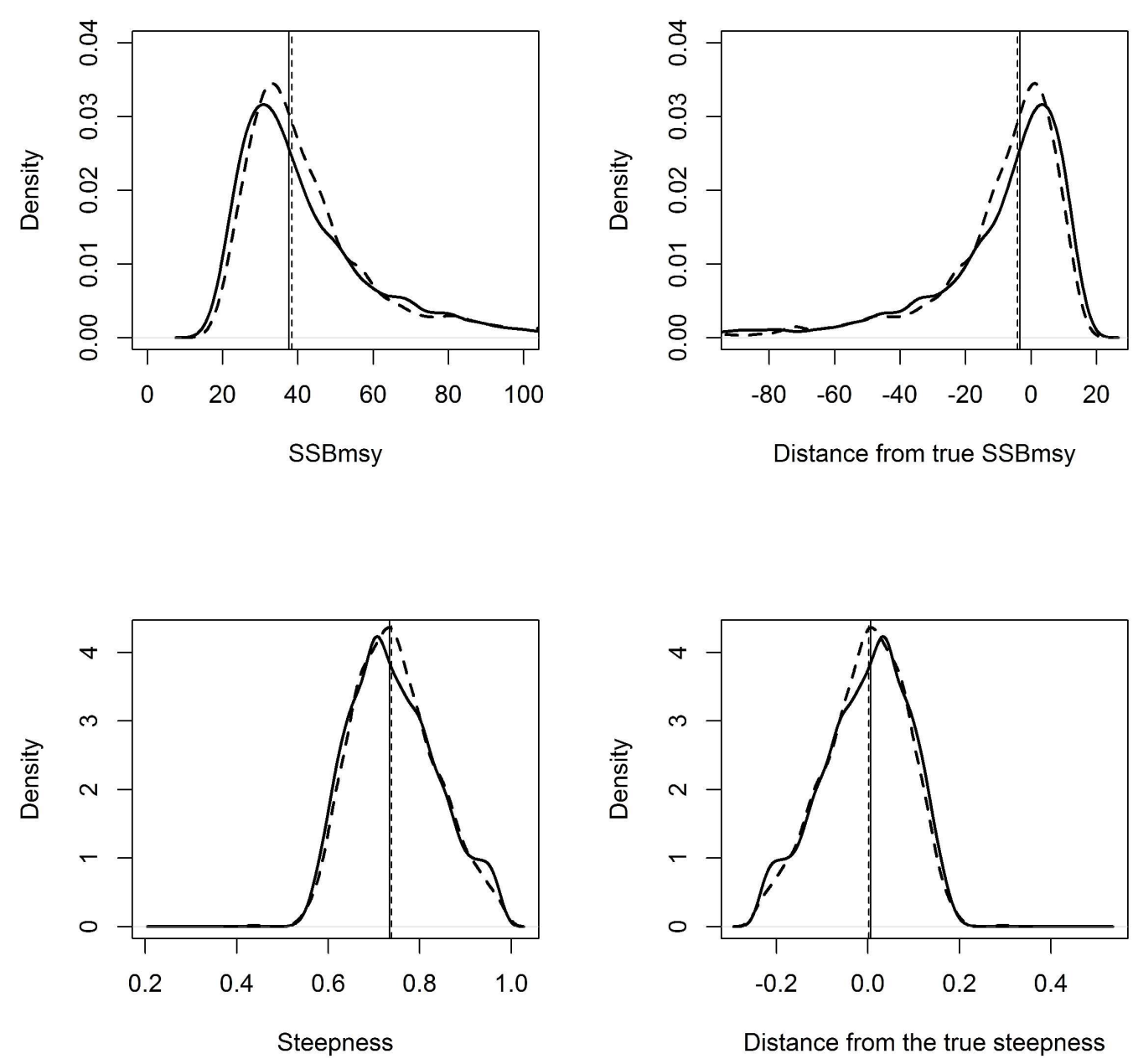

Distance from the true steepness 
Supplementary Material

Figure S38. All compositions (increased sample size)
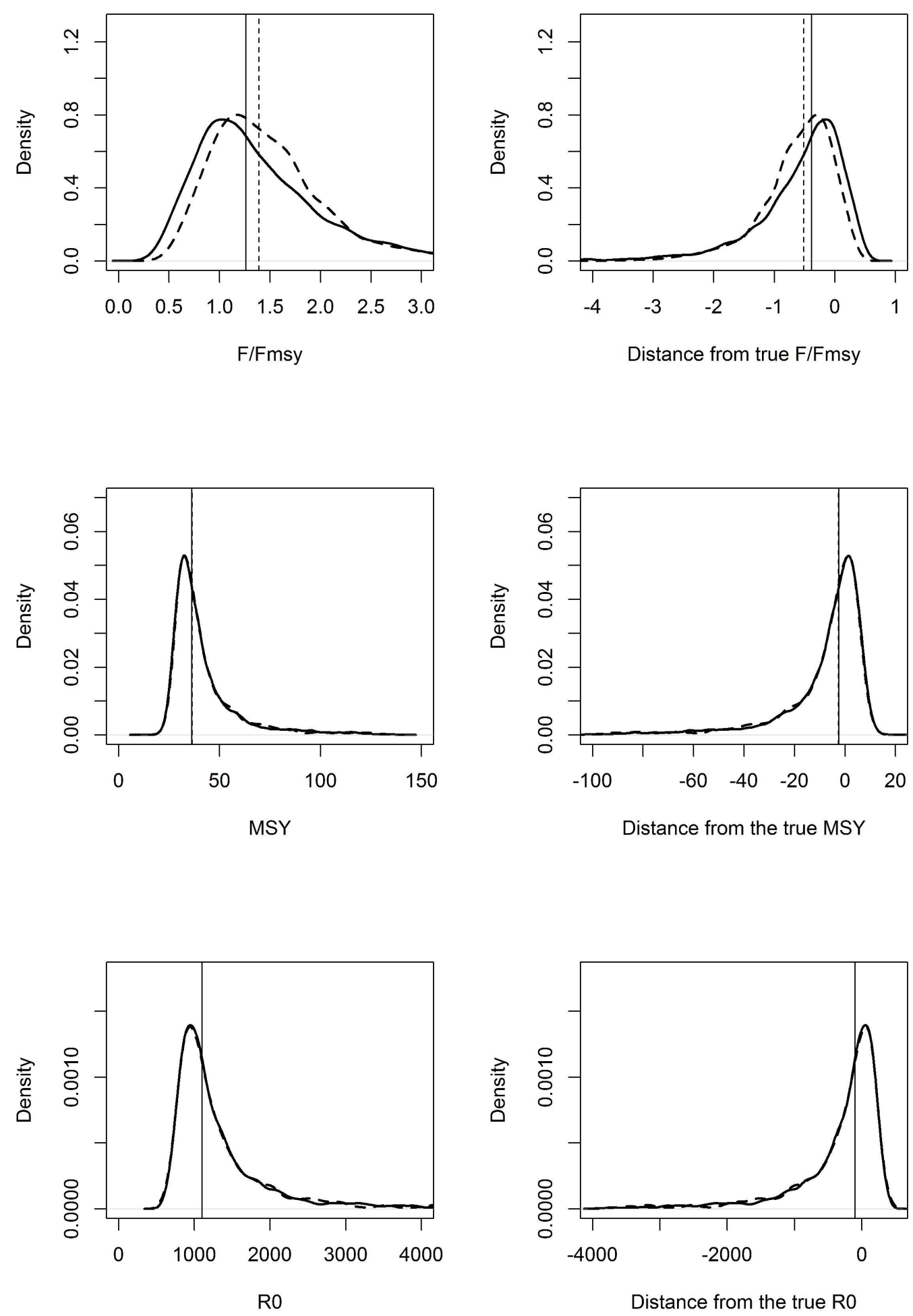
Supplementary Material

Figure S39. All compositions (increased sample size)
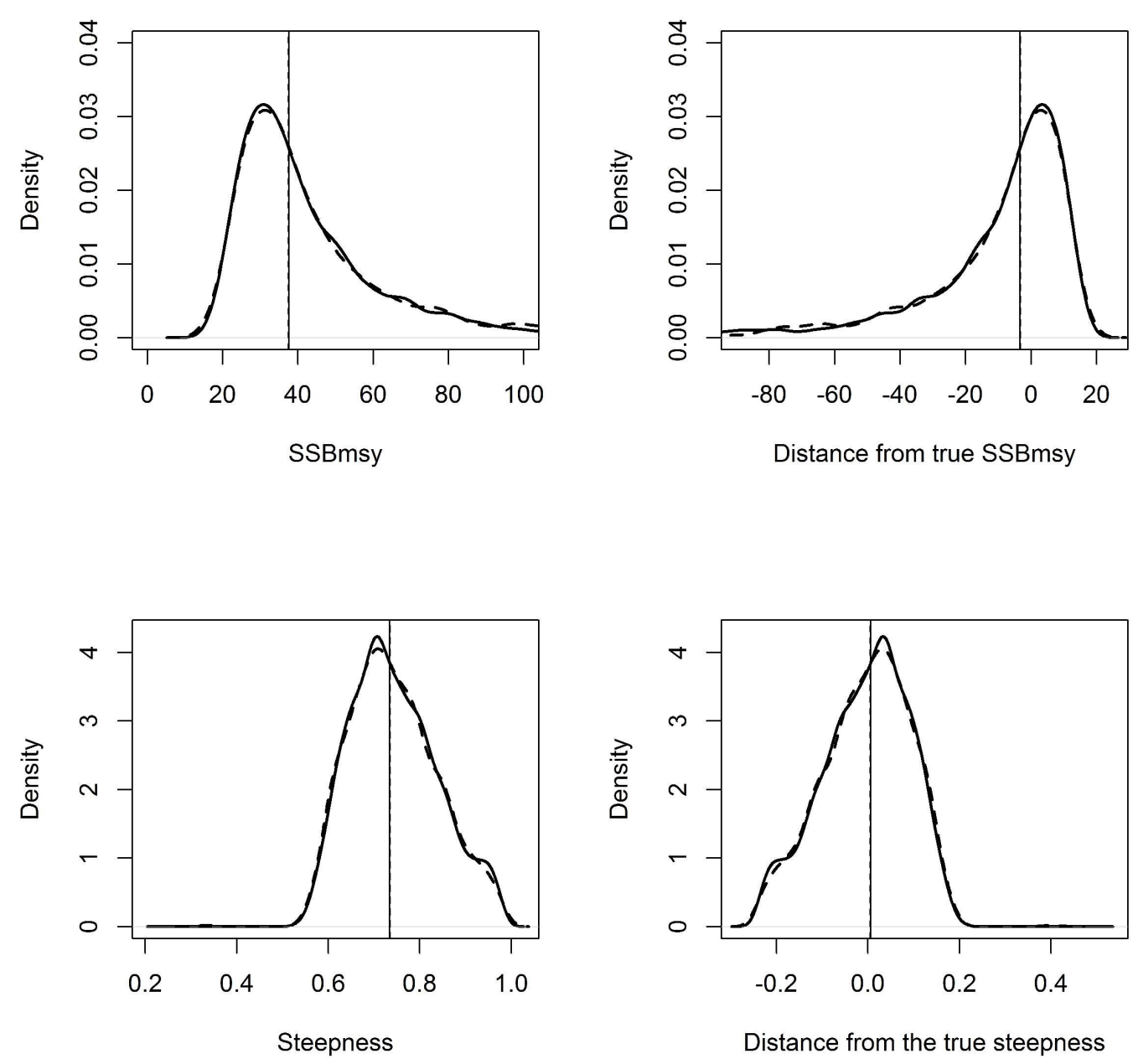

Distance from the true steepness 
Supplementary Material

Figure S40. Landings and discards
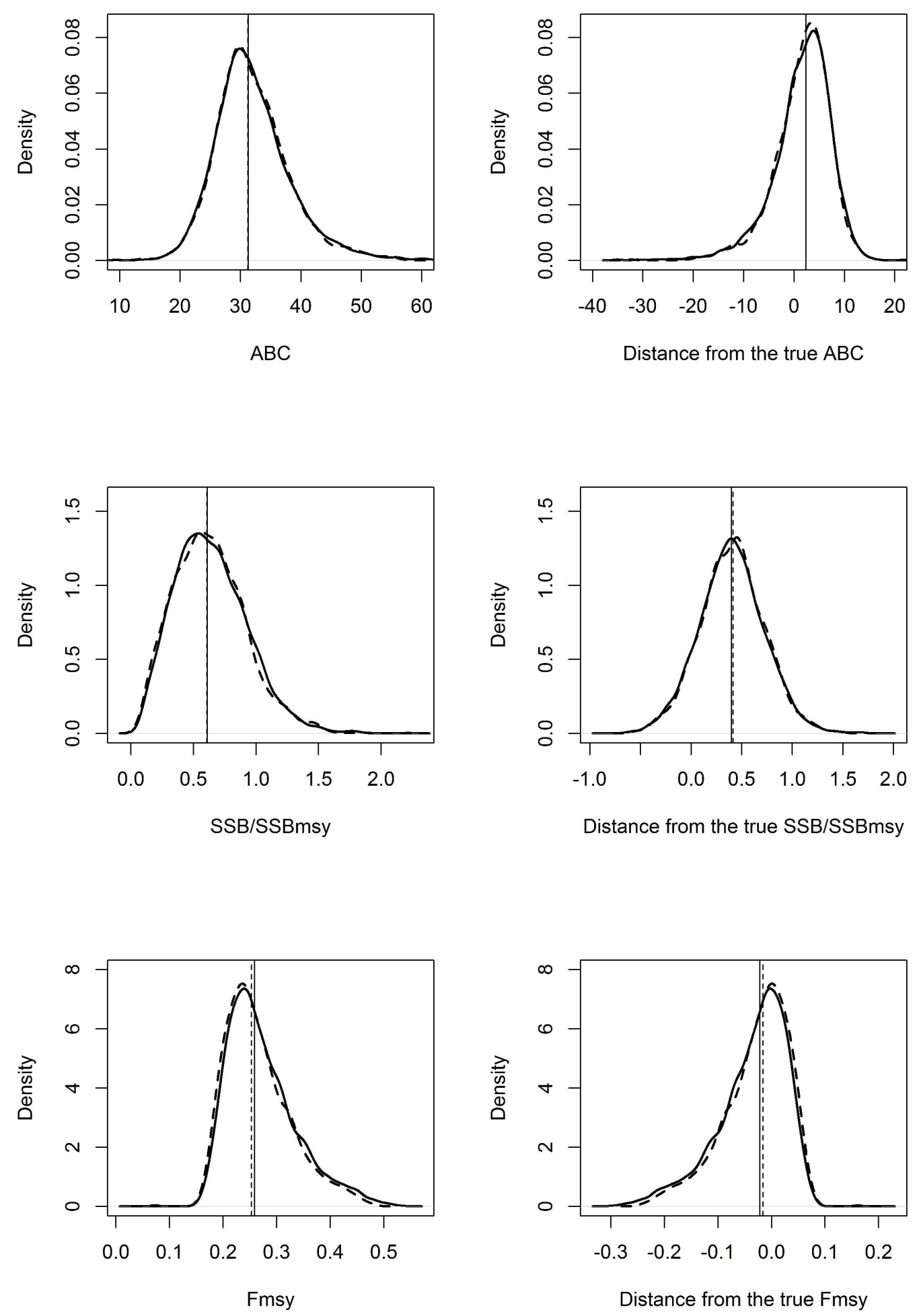
Supplementary Material

Figure S41. Landings and discards
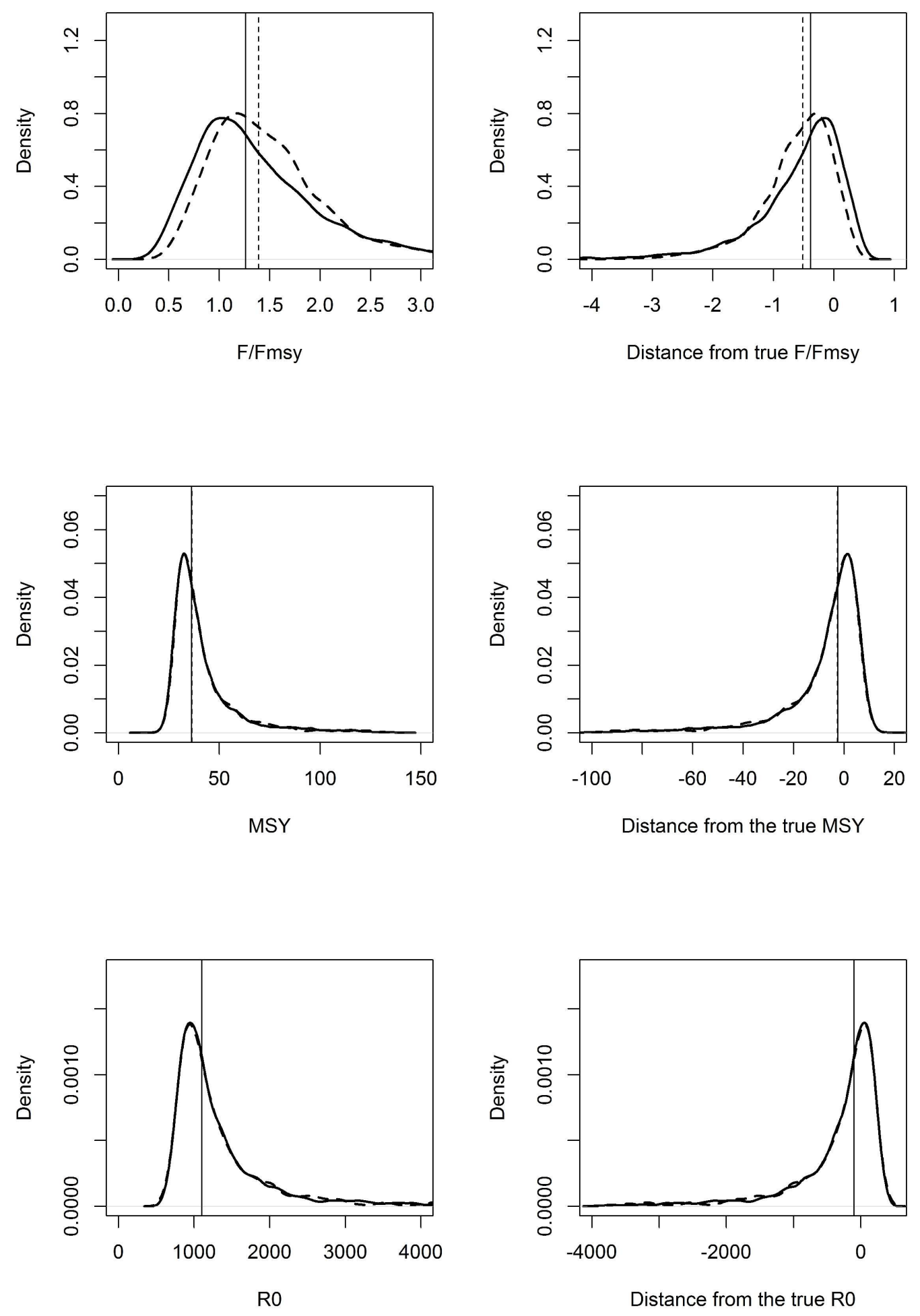
Supplementary Material

Figure S42. Landings and discards
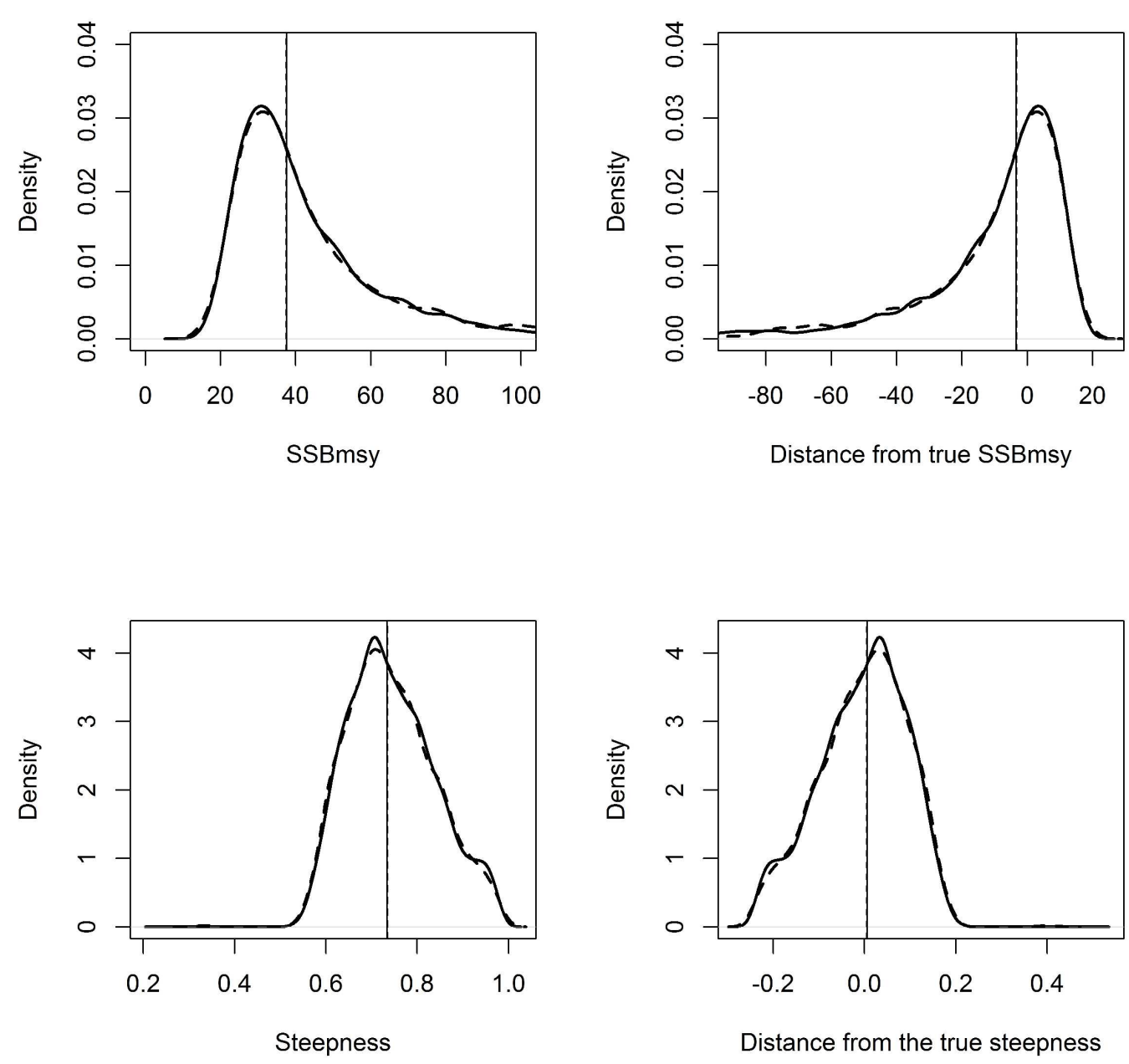

Distance from the true steepness 


\section{Supplementary Material}

BAM .tpl file

\section{Operating model code}

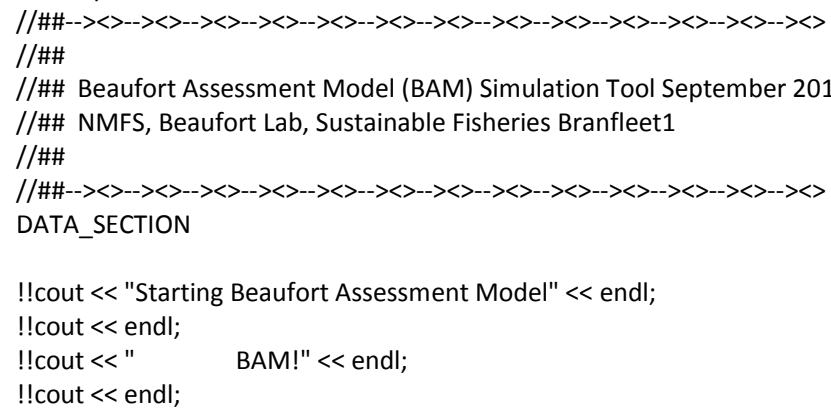

// Starting and ending year of the model (year data starts) init_int styr;

init_int endyr;

//Starting year to estimate recruitment deviation from S-R curve init_int styr_rec_dev;

//Ending year to estimate recruitment deviation from S-R curve

init_int endyr_rec_dev;

//possible 3 phases of constraints on recruitment deviations

init_int endyr_rec_phase1;

init_int endyr_rec_phase2;

// ending years for selectivity blocks

init_int endyr_selex_phase1;

init_int endyr_selex_phase2;

//number assessment years

number nyrs;

number nyrs_rec;

//this section MUST BE INDENTED!!!

LOCAL_CALCS

nyrs=endyr-styr+1.;

nyrs_rec=endyr_rec_dev-styr_rec_dev+1.;

END_CALCS

//Total number of ages in population model init_int nages;

$/ /$ Vector of ages for age bins in population model init_vector agebins(1,nages);

//Max F used in spr and msy calcs init_number max_F_spr_msy;

//Total number of iterations for spr calcs init_int n_iter_spr;

//Total number of iterations for msy calcs init_int n_iter_msy;

//Number years at end of time series over whifleet1 to average sector F's, for weighted selectivities init_int selpar_n_yrs_wgted;

//bias correction (set to 1.0 for no bias correction or a negative value to compute from rec variance) init_number set_BiasCor;

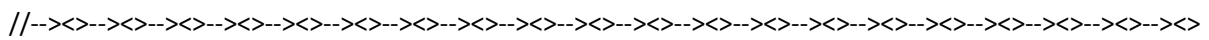




\section{Supplementary Material}

//-- BAM DATA_SECTION: observed data section

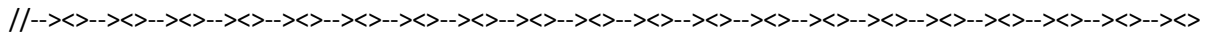

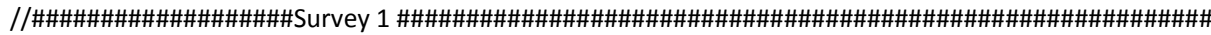
//CPUE: Survey1

init_int styr_survey1_cpue;

init_int endyr_survey1_cpue;

init_vector obs_survey1_cpue(styr_survey1_cpue,endyr_survey1_cpue);//Observed CPUE

init_vector survey1_cpue_cv(styr_survey1_cpue,endyr_survey1_cpue); //CV of cpue

// Survey1 Age Compositions

init_int nyr_survey1_agec;

init_ivectoryrs_survey1_agec(1,nyr_survey1_agec);

init_vectornsamp_survey1_agec(1,nyr_survey1_agec);

init_vector nfish_survey1_agec(1,nyr_survey1_agec);

init_matrix obs_survey1_agec(1,nyr_survey1_agec,1,nages);

//\#\#\#\#\#\#\#\#\#\#\#\#\#Fleet1 \#\#\#\#\#\#\#\#\#\#\#\#\#\#\#\#\#\#\#\#\#\#\#\#\#\#\#\#\#\#\#

//CPUE: fleet1

init_int styr_fleet1_cpue;

init_int endyr_fleet1_cpue;

init_vector obs_fleet1_cpue(styr_fleet1_cpue,endyr_fleet1_cpue); //Observed CPUE

init_vector fleet1_cpue_cv(styr_fleet1_cpue,endyr_fleet1_cpue); //CV of cpue

\section{// Landings}

init_int styr_fleet1_L;

init_int endyr_fleet1_L;

init_vector obs_fleet1_L(styr_fleet1_L,endyr_fleet1_L);

init_vector fleet1_L_cv(styr_fleet1_L,endyr_fleet1_L);

// Discards

init_int styr_fleet1_D;

init_int endyr_fleet1_D;

init_vector obs_fleet1_D(styr_fleet1_D,endyr_fleet1_D);

init_vector fleet1_D_cv(styr_fleet1_D,endyr_fleet1_D);

// Fleet1 age compositions

init_int nyr_fleet1_agec;

init_ivector yrs_fleet1_agec(1,nyr_fleet1_agec);

init_vector nsamp_fleet1_agec(1,nyr_fleet1_agec);

init_vector nfish_fleet1_agec(1,nyr_fleet1_agec);

init_matrix obs_fleet1_agec(1,nyr_fleet1_agec,1,nages);

//\#\#\#\#\#\#\#\#\#\#\#\#\#\#Fleet2 \#\#\#\#\#\#\#\#\#\#\#\#\#\#\#\#\#\#\#\#\#\#\#\#\#\#\#\#\#\#\#\#\#

// Landings

init_int styr_fleet2_L;

init_int endyr_fleet2_L;

init_vector obs_fleet2_L(styr_fleet2_L,endyr_fleet2_L);

init_vector fleet2_L_cv(styr_fleet2_L,endyr_fleet2_L);

// Discards

init_int styr_fleet2_D;

init_int endyr_fleet2_D;

init_vector obs_fleet2_D(styr_fleet2_D,endyr_fleet2_D);

init_vector fleet2_D_cv(styr_fleet2_D,endyr_fleet2_D);

// Fleet2 age compositions

init_int nyr_fleet2_agec;

init_ivector yrs_fleet2_agec(1,nyr_fleet2_agec);

init_vector nsamp_fleet2_agec(1,nyr_fleet2_agec);

init_vector nfish_fleet2_agec(1,nyr_fleet2_agec);

init_matrix obs_fleet2_agec(1,nyr_fleet2_agec,1,nages); 


\section{Supplementary Material}

//-- BAM DATA_SECTION: parameter section

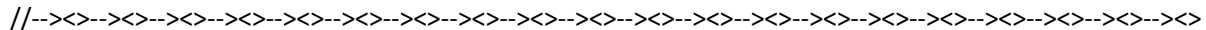

//\#\#\#\#\#\#\#\#\#\#\#\#\#\#\#\#Single Parameter values and initial guesses \#\#\#\#\#\#\#\#\#\#\#\#\#\#\#\#\#\#\#\#\#\#\#\#\#\#\#\# // Von Bert parameters in TL mm all fish init_vector set_Linf(1,7);

init_vector set_K(1,7);

init_vector set_tO(1,7);

//Spawner-recruit parameters (Initial guesses or fixed values)

init_vector set_steep $(1,7)$; //recruitment steepness

init_vector set_log_RO $(1,7)$; //recruitment R0

init_vector set_R_autocorr(1,7); //recruitment autocorrelation

init_vector set_rec_sigma(1,7); //recruitment standard deviation in log space

//Initial guesses or fixed values of estimated selectivity parameters

init_vector set_selpar_L50_survey1 $(1,7)$;

init_vector set_selpar_slope_survey $1(1,7)$;

init_vector set_selpar_L50_fleet1(1,7);

init_vector set_selpar_slope_fleet1 $(1,7)$;

//init_vector set_selpar_L50_fleet1_D(1,7);

//init_vector set_selpar_slope_fleet1_D(1,7);

//init_vector set_selpar_L502_fleet1_D(1,7);

//init_vector set_selpar_slope2_fleet1_D(1,7);

init_vector set_selpar_peak_fleet1_D(1,7);

init_vector set_selpar_top_fleet1_D(1,7);

init_vector set_selpar_ascwid_fleet1_D(1,7);

init_vector set_selpar_deswid_fleet1_D(1,7);

init_vector set_selpar_init_fleet1_D(1,7);

init_vector set_selpar_final_fleet1_D(1,7);

init_vector set_selpar_L50_fleet2 $(1,7)$;

init_vector set_selpar_slope_fleet2 $(1,7)$;

//init_vector set_selpar_L50_fleet2_D(1,7);

//init_vector set_selpar_slope_fleet2_D(1,7);

//init_vector set_selpar_L502_fleet2_D(1,7);

//init_vector set_selpar_slope2_fleet2_D(1,7);

init_vector set_selpar_peak_fleet2_D(1,7);

init_vector set_selpar_top_fleet2_D(1,7);

init_vector set_selpar_ascwid_fleet2_D(1,7);

init_vector set_selpar_deswid_fleet2_D(1,7);

init_vector set_selpar_init_fleet2_D(1,7);

init_vector set_selpar_final_fleet2_D(1,7);

//--index catfleet1ability

init_vector set_log_q_survey1(1,7); //catchability coefficient $(\log )$ for survey1 cpue index

init_vector set_log_q_fleet1(1,7); //catchability coefficient (log) for fleet1 cpue index

//initial F

init_vector set_F_init(1,7); //scales initial F

//--mean F's in log space

init_vector set_log_avg_F_fleet1 $(1,7)$;

init_vector set_log_avg_F_fleet1_D(1,7);

init_vector set_log_avg_F_fleet2 $(1,7)$;

init_vector set_log_avg_F_fleet2_D(1,7);

//\#\#\#\#\#\#\#\#\#\#\#\#\#\#\#\#粮 Vector Parameter values (vals) and bounds \#\#\#\#\#\#\#\#\#\#\#\#\#\#\#\#\#\#\#\#\#\#\#\#\#\#\#

//--F vectors-

init_vector set_log_F_dev_fleet1(1,3);

init_vector set_log_F_dev_fleet1_D(1,3);

init_vector set_log_F_dev_fleet2(1,3);

init_vector set_log_F_dev_fleet2_D(1,3); 


\section{Supplementary Material}

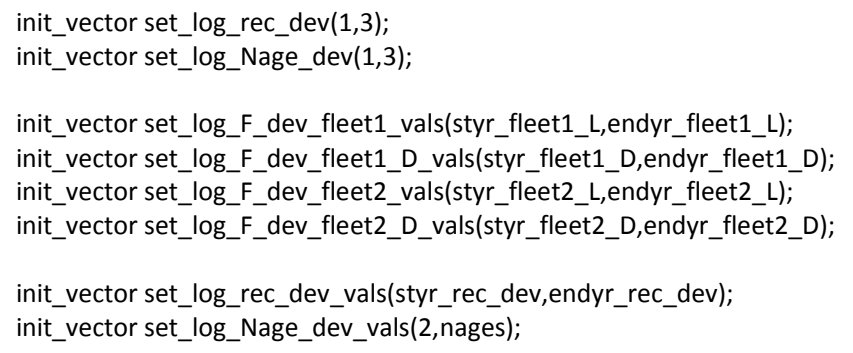




\title{
Supplementary Material
}

\author{
//Tune Fapex (tuning removed in final year of optimization) \\ init number set Ftune; \\ init_int set_Ftune_yr; \\ //threshold sample sizes for age comps \\ init_number minSS_survey1_agec; \\ init_number minSS_fleet1_agec; \\ init_number minSs_fleet2_agec;
}

//ageing error matrix (columns are true ages, rows are ages as read for age comps: columns should sum to one) init_matrix age_error(1,nages,1,nages);

// \#\#\#\#\#\#Indexing integers for year(iyear), age(iage),length(ilen) \#\#\#\#\#\#\#\#\#\#\#\#\#

int iyear;

int iage;

int ilen;

int ff;

number sqrt2pi;

number g2mt

//conversion of grams to metric tons

number g2 kg;

number g2klb;

//conversion of grams to $\mathrm{kg}$

//conversion of grams to $1000 \mathrm{lb}$

number $\mathrm{mt} 2 \mathrm{klb}$

//conversion of metric tons to $1000 \mathrm{lb}$

number mt2lb;

//conversion of metric tons to $\mathrm{lb}$

number dzero;

//small additive constant to prevent division by zero

number huge_number; $\quad$ //huge number, to avoid irregular parameter space

init_number end_of_data_file;

//this section MUST BE INDENTED!!!

LOCAL_CALCS

if(end_of_data_file!=999)

\{

cout $<<" * * *$ WARNING: Data File NOT READ CORRECTLY $* * * * "<<$ endl; exit(0);

else

\{cout $<<$ "Data File read correctly" << endl;

END_CALCS

PARAMETER_SECTION

LOCAL_CALCS

const double dummy_optimize_PH=set_dummy_optimize;

const double Linf_LO=set_Linf(2); const double Linf_HI=set_Linf(3); const double Linf_PH=set_Linf(4);

const double K_LO=set_K(2); const double K_HI=set_K(3); const double K_PH=set_K(4);

const double t0_LO=set_tO(2); const double t0_HI=set_tO(3); const double t0_PH=set_tO(4);

const double steep_LO=set_steep(2); const double steep_HI=set_steep(3); const double steep_PH=set_steep(4);

const double log_RO_LO=set_log_RO(2); const double log_RO_HI=set_log_RO(3); const double log_RO_PH=set_log_RO(4);

const double R_autocorr_LO=set_R_autocorr(2); const double R_autocorr_HI=set_R_autocorr(3); const double

R_autocorr_PH=set_R_autocorr(4);

const double rec_sigma_LO=set_rec_sigma(2); const double rec_sigma_HI=set_rec_sigma(3); const double rec_sigma_PH=set_rec_sigma(4);

const double selpar_L50_survey1_LO=set_selpar_L50_survey1(2); const double selpar_L50_survey1_HI=set_selpar_L50_survey1(3); const double selpar_L50_survey1_PH=set_selpar_L50_survey1(4);

const double selpar_slope_survey1_LO=set_selpar_slope_survey1(2); const double selpar_slope_survey1_HI=set_selpar_slope_survey1(3); const double selpar_slope_survey1_PH=set_selpar_slope_survey1(4);

const double selpar_L50_fleet1_LO=set_selpar_L50_fleet1(2); const double selpar_L50_fleet1_HI=set_selpar_L50_fleet1(3); const double selpar_L50_fleet1_PH=set_selpar_L50_fleet1(4);

const double selpar_slope_fleet1_LO=set_selpar_slope_fleet1(2); const double selpar_slope_fleet1_HI=set_selpar_slope_fleet1(3); const double selpar_slope_fleet1_PH=set_selpar_slope_fleet1(4);

//const double selpar_L50_fleet1____LO=set_selpar_L50_fleet1_D(2); const double selpar_L50_fleet1_D_HI=set_selpar_L50_fleet1_D(3); const double selpar_L50_fleet1_D_PH=set_selpar_L50_fleet1_D(4); 


\section{Supplementary Material}

//const double selpar_slope_fleet1_D_LO=set_selpar_slope_fleet1_D(2); const double
selpar_slope_fleet1_D_HI=set_selpar_slope_fleet1_D(3); const double selpar_slope_fleet1_D_PH=set_selpar_slope_fleet1_D(4);

//const double selpar_L502_fleet1_D_LO=set_selpar_L502_fleet1_D(2); const double selpar_L502_fleet1_D_HI=set_selpar_L502_fleet1_D(3); const double selpar_L502_fleet1_D_PH=set_selpar_L502_fleet1_D(4);

//const double selpar_slope2_fleet1_D_LO=set_selpar_slope2_fleet1_D(2); const double

selpar_slope2_fleet1_D_HI=set_selpar_slope2_fleet1_D(3); const double selpar_slope2_fleet1_D_PH=set_selpar_slope2_fleet1_D(4);

const double selpar_peak_fleet1_D_LO=set_selpar_peak_fleet1_D(2); const double selpar_peak_fleet1_D_HI=set_selpar_peak_fleet1_D(3); const double selpar_peak_fleet1_D_PH=set_selpar_peak_fleet1_D(4);

const double selpar_top_fleet1_D_LO=set_selpar_top_fleet1_D(2); const double selpar_top_fleet1_D_HI=set_selpar_top_fleet1_D(3); const double selpar_top_fleet1_D_PH=set_selpar_top_fleet1_D(4);

const double selpar_ascwid_fleet1_D_LO=set_selpar_ascwid_fleet1_D(2); const double

selpar_ascwid_fleet1_D_HI=set_selpar_ascwid_fleet1_D(3); const double selpar_ascwid_fleet1_D_PH=set_selpar_ascwid_fleet1_D(4);

const double selpar_deswid_fleet1_D_LO=set_selpar_deswid_fleet1_D(2); const double

selpar_deswid_fleet1_D_HI=set_selpar_deswid_fleet1_D(3); const double selpar_deswid_fleet1_D_PH=set_selpar_deswid_fleet1_D(4);

const double selpar_init_fleet1_D_LO=set_selpar_init_fleet1_D(2); const double selpar_init_fleet1_D_HI=set_selpar_init_fleet1_D(3); const double selpar_init_fleet1_D_PH=set_selpar_init_fleet1_D(4);

const double selpar_final_fleet1_D_LO=set_selpar_final_fleet1_D(2); const double selpar_final_fleet1_D_HI=set_selpar_final_fleet1_D(3); const double selpar_final_fleet1_D_PH=set_selpar_final_fleet1_D(4);

const double selpar_L50_fleet2_LO=set_selpar_L50_fleet2(2); const double selpar_L50_fleet2_HI=set_selpar_L50_fleet2(3); const double selpar_L50_fleet2_PH=set_selpar_L50_fleet2(4);

const double selpar_slope_fleet2_LO=set_selpar_slope_fleet2(2); const double selpar_slope_fleet2_HI=set_selpar_slope_fleet2(3); const double selpar_slope_fleet2_PH=set_selpar_slope_fleet2(4);

//const double selpar_L50_fleet2_D_LO=set_selpar_L50_fleet2_D(2); const double selpar_L50_fleet2_D_HI=set_selpar_L50_fleet2_D(3); const double selpar_L50_fleet2_D_PH=set_selpar_L50_fleet2_D(4);

//const double selpar_slope_fleet2_D_LO=set_selpar_slope_fleet2_D(2); const double

selpar_slope_fleet2_D_HI=set_selpar_slope_fleet2_D(3); const double selpar_slope_fleet2_D_PH=set_selpar_slope_fleet2_D(4);

//const double selpar_L502_fleet2_D_LO=set_selpar_L502_fleet2_D(2); const double selpar_L502_fleet2_D_HI=set_selpar_L502_fleet2_D(3); const double selpar_L502_fleet2_D_PH=set_selpar_L502_fleet2_D(4);

//const double selpar_slope2_fleet2_D_LO=set_selpar_slope2_fleet2_D(2); const double

selpar_slope2_fleet2_D_HI=set_selpar_slope2_fleet2_D(3); const double selpar_slope2_fleet2_D_PH=set_selpar_slope2_fleet2_D(4);

const double selpar_peak_fleet2_D_LO=set_selpar_peak_fleet2_D(2); const double selpar_peak_fleet2_D_HI=set_selpar_peak_fleet2_D(3); const double selpar_peak_fleet2_D_PH=set_selpar_peak_fleet2_D(4);

const double selpar_top_fleet2_D_LO=set_selpar_top_fleet2_D(2); const double selpar_top_fleet2_D_HI=set_selpar_top_fleet2_D(3); const double selpar_top_fleet2_D_PH=set_selpar_top_fleet2_D(4);

const double selpar_ascwid_fleet2_D_LO=set_selpar_ascwid_fleet2_D(2); const double

selpar_ascwid_fleet2_D_HI=set_selpar_ascwid_fleet2_D(3); const double selpar_ascwid_fleet2_D_PH=set_selpar_ascwid_fleet2_D(4);

const double selpar_deswid_fleet2_D_LO=set_selpar_deswid_fleet2_D(2); const double

selpar_deswid_fleet2_D_HI=set_selpar_deswid_fleet2_D(3); const double selpar_deswid_fleet2_D_PH=set_selpar_deswid_fleet2_D(4);

const double selpar_init_fleet2_D_LO=set_selpar_init_fleet2_D(2); const double selpar_init_fleet2_D_HI=set_selpar_init_fleet2_D(3); const double selpar_init_fleet2_D_PH=set_selpar_init_fleet2_D(4);

const double selpar_final_fleet2_D_LO=set_selpar_final_fleet2_D(2); const double selpar_final_fleet2_D_HI=set_selpar_final_fleet2_D(3); const double selpar_final_fleet2_D_PH=set_selpar_final_fleet2_D(4);

const double log_q_survey1_LO=set_log_q_survey1(2); const double log_q_survey1_HI=set_log_q_survey1(3); const double

log_q_survey1_PH=set_log_q_survey1(4);

const double log_q_fleet1_LO=set_log_q_fleet1(2); const double log_q_fleet1_HI=set_log_q_fleet1(3); const double

log_q_fleet1_PH=set_log_q_fleet1(4);

const double F_init_LO=set_F_init(2); const double F_init_HI=set_F_init(3); const double F_init_PH=set_F_init(4);

const double log_avg_F_fleet1_LO=set_log_avg_F_fleet1(2); const double log_avg_F_fleet1_HI=set_log_avg_F_fleet1(3); const double

log_avg_F_fleet1_PH=set_log_avg_F_fleet1(4);

const double log_avg_F_fleet1_D_LO=set_log_avg_F_fleet1_D(2); const double log_avg_F_fleet1_D_HI=set_log_avg_F_fleet1_D(3); const double log_avg_F_fleet1_D_PH=set_log_avg_F_fleet1_D(4);

const double log_avg_F_fleet2_LO=set_log_avg_F_fleet2(2); const double log_avg_F_fleet2_HI=set_log_avg_F_fleet2(3); const double log_avg_F_fleet2_PH=set_log_avg_F_fleet2(4);

const double log_avg_F_fleet2_D_LO=set_log_avg_F_fleet2_D(2); const double log_avg_F_fleet2_D_HI=set_log_avg_F_fleet2_D(3); const double log_avg_F_fleet2_D_PH=set_log_avg_F_fleet2_D(4);

//-dev vectors-

const double log_F_dev_fleet1_LO=set_log_F_dev_fleet1(1); const double log_F_dev_fleet1_HI=set_log_F_dev_fleet1(2); const double log_F_dev_fleet1_PH=set_log_F_dev_fleet1(3);

const double log_F_dev_fleet1_D_LO=set_log_F_dev_fleet1_D(1); const double log_F_dev_fleet1_D_HI=set_log_F_dev_fleet1_D(2); const double log_F_dev_fleet1_D_PH=set_log_F_dev_fleet1_D(3);

const double log_F_dev_fleet2_LO=set_log_F_dev_fleet2(1); const double log_F_dev_fleet2_HI=set_log_F_dev_fleet2(2); const double log_F_dev_fleet2_PH=set_log_F_dev_fleet2(3); 


\section{Supplementary Material}

const double log_F_dev_fleet2_D_LO=set_log_F_dev_fleet2_D(1); const double log_F_dev_fleet2_D_HI=set_log_F_dev_fleet2_D(2); const double log_F_dev_fleet2_D_PH=set_log_F_dev_fleet2_D(3);

const double log_rec_dev_LO=set_log_rec_dev(1); const double log_rec_dev_HI=set_log_rec_dev(2); const double log_rec_dev_PH=set_log_rec_dev(3);

const double log_Nage_dev_LO=set_log_Nage_dev(1); const double log_Nage_dev_HI=set_log_Nage_dev(2); const double log_Nage_dev_PH=set_log_Nage_dev(3);

\section{END_CALCS}

//-----dummy optimization parameter

init_number dummy_optimize(dummy_optimize_PH);

////-----------Growth-

init_bounded_number Linf(Linf_LO,Linf_HI,Linf_PH);

init_bounded_number K(K_LO,K_HI,K_PH);

init_bounded_number tO(tO_LO,tO_HI,tO_PH);

vector Linf_out(1,8);

vector $\mathrm{K}$ out $(1,8)$;

vector t0_out $(1,8)$;

vector meanlen_TL(1,nages); //mean total length $(\mathrm{mm})$ at age all fish

vector wgt_g(1,nages); //whole wgt in $\mathrm{g}$

vector wgt_kg(1,nages); //whole wgt in $\mathrm{kg}$

vector wgt_mt(1,nages); //whole wgt in $\mathrm{mt}$

vector wgt_klb(1,nages); //whole wgt in $1000 \mathrm{lb}$

vector wgt_lb(1,nages); //whole wgt in $\mathrm{lb}$

matrix wholewgt_fleet1_klb(styr,endyr,1,nages); //whole wgt of fleet1 landings in $1000 \mathrm{lb}$ matrix wholewgt_fleet2_klb(styr,endyr,1,nages); //whole wgt of fleet2 landings in $1000 \mathrm{lb}$ matrix wholewgt_fleet1_D_klb(styr,endyr,1,nages); //whole wgt of fleet1 landings in $1000 \mathrm{lb}$ matrix wholewgt_fleet2_D_klb(styr,endyr,1,nages); //whole wgt of fleet2 landings in $1000 \mathrm{lb}$

\section{//----Predicted age compositions}

matrix pred_survey1_agec(1,nyr_survey1_agec,1,nages);

matrix pred_survey1_agec_allages(1,nyr_survey1_agec,1,nages);

matrix ErrorFree_survey1_agec(1,nyr_survey1_agec,1,nages);

matrix pred_fleet1_agec(1,nyr_fleet1_agec,1,nages);

matrix pred_fleet1_agec_allages(1,nyr_fleet1_agec,1,nages);

matrix ErrorFree_fleet1_agec(1,nyr_fleet1_agec,1,nages);

matrix pred_fleet2_agec(1,nyr_fleet2_agec,1,nages);

matrix pred_fleet2_agec_allages(1,nyr_fleet2_agec,1,nages);

matrix ErrorFree_fleet2_agec(1,nyr_fleet2_agec,1,nages);

//effective sample size applied in multinomial distributions

vector nsamp_survey1_agec_allyr(styr,endyr);

vector nsamp_fleet1_agec_allyr(styr,endyr);

vector nsamp_fleet2_agec_allyr(styr,endyr);

//Nfish used in MCB analysis (not used in fitting) vector nfish_survey1_agec_allyr(styr,endyr); vector nfish_fleet1_agec_allyr(styr,endyr); vector nfish_fleet2_agec_allyr(styr,endyr);

//Computed effective sample size for output (not used in fitting) vector neff_survey1_agec_allyr_out(styr,endyr); vector neff_fleet1_agec_allyr_out(styr,endyr); vector neff_fleet2_agec_allyr_out(styr,endyr);

//-----Population-

matrix N(styr, endyr+1,1,nages). //Population numbers by year and age at start of $y \mathrm{r}$

matrix N_mdyr(styr,endyr,1,nages); //Population numbers by year and age at mdpt of yr: used for comps and cpue matrix N_spawn(styr,endyr,1,nages); //Population numbers by year and age at peaking spawning: used for SSB vector $\mathrm{N} \_$proj(1,nages); 


\title{
Supplementary Material
}

\author{
init_bounded_vector log_Nage_dev(2,nages,log_Nage_dev_LO,log_Nage_dev_HI,log_Nage_dev_PH); \\ vector log_Nage_dev_output(1,nages); //used in output. equals zero for first age \\ matrix $\mathrm{B}$ (styr,endyr+1,1,nages); //Population biomass by year and age at start of $\mathrm{yr}$ \\ vector totB(styr,endyr+1); $\quad / / /$ Total biomass by year \\ vector totN(styr,endyr+1); $\quad$ //Total abundance by year \\ vector SSB(styr,endyr); $\quad$ //Total spawning biomass by year (female + male mature biomass) \\ vector MatFemB(styr,endyr); //Total spawning biomass by year (mature female biomass) \\ vector rec(styr,endyr+1); $\quad / /$ Recruits by year \\ vector prop_f(1,nages); $\quad$ //Proportion female by age, all males assumed mature \\ vector maturity_f(1,nages); $\quad / / P r o p o r t i o n$ of female mature at age \\ vector reprod(1,nages); $\quad$ //vector used to compute spawning biomass (total mature biomass - males + females)
}

//---Stock-Recruit Function (Beverton-Holt, steepness parameterization)--------init_bounded_number log_RO(log_RO_LO,log_RO_HI,log_RO_PH); //log(virgin Recruitment) vector log_RO_out $(1,8)$; number RO; $\quad$ //virgin recruitment init_bounded_number steep(steep_LO,steep_HI,steep_PH); //steepness vector steep_out(1,8);

init_bounded_number rec_sigma(rec_sigma_LO,rec_sigma_HI,rec_sigma_PH); //sd recruitment residuals //KC comment out; this should fix it at the initial value vector rec_sigma_out(1,8); init_bounded_number R_autocorr(R_autocorr_LO,R_autocorr_HI,R_autocorr_PH); //autocorrelation in SR KC commented out since not estimated vector R_autocorr_out(1,8);

number rec_sigma_sq; $\quad$ //square of rec_sigma

number rec_logL_add; $\quad$ //additive term in - $\log L$ term

//vector log_rec_dev(styr_rec_dev,endyr_rec_dev);

init_bounded_dev_vector log_rec_dev(styr_rec_dev,endyr_rec_dev,log_rec_dev_LO,log_rec_dev_HI,log_rec_dev_PH); vector log_rec_dev_output(styr,endyr+1); //used in t.series output. equals zero except for yrs in log_rec_dev vector log_rec_dev_out(styr_rec_dev,endyr_rec_dev); //used in output for bound fleet1ecking

numbervar_rec_dev; number sigma_rec_dev;

number BiasCor;

number S0;

number BO;

number R1;

number R_virgin;

vector SdSO(styr,endyr);
$/ /$ variance of log recruitment deviations, from yrs with unconstrainted $S-R(X X X X-X X X X)$

//sample SD of log residuals (may not equal rec_sigma

//Bias correction in equilibrium recruits

//equal to spr_FO*RO $=$ virgin SSB

//equal to bpr_FO*RO = virgin $\mathrm{B}$

//Recruits in styr

//unfished recruitment with bias correction

//SSB / virgin SSB

$/ /-$

////---Selectivity-

//survey1

matrix sel_survey1(styr,endyr,1,nages);

init_bounded_number selpar_L50_survey1(selpar_L50_survey1_LO,selpar_L50_survey1_HI,selpar_L50_survey1_PH);

init_bounded_number selpar_slope_survey1(selpar_slope_survey1_LO,selpar_slope_survey1_HI,selpar_slope_survey1_PH);

vector selpar_L50_survey1_out $(1,8)$;

vector selpar_slope_survey1_out(1,8);

//fleet1 landings--

matrix sel_fleet1(styr,endyr,1,nages);

init_bounded_number selpar_L50_fleet1(selpar_L50_fleet1_LO,selpar_L50_fleet1_HI,selpar_L50_fleet1_PH);

init_bounded_number selpar_slope_fleet1(selpar_slope_fleet1_LO,selpar_slope_fleet1_HI,selpar_slope_fleet1_PH);

vector selpar_L50_fleet1_out(1,8);

vector selpar_slope_fleet1_out(1,8);

//fleet1 discards--

matrix sel_fleet1_D(styr,endyr,1,nages);

//init_bounded_number selpar_L50_fleet1_D(selpar_L50_fleet1_D_LO,selpar_L50_fleet1_D_HI,selpar_L50_fleet1_D_PH);

//init_bounded_number selpar_slope_fleet1_D(selpar_slope_fleet1_D_LO,selpar_slope_fleet1_D_HI,selpar_slope_fleet1_D_PH);

//init_bounded_number selpar_L502_fleet1_D(selpar_L502_fleet1_D_LO,selpar_L502_fleet1_D_HI,selpar_L502_fleet1_D_PH); 


\section{Supplementary Material}

//init_bounded_number selpar_slope2_fleet1_D(selpar_slope2_fleet1_D_LO,selpar_slope2_fleet1_D_HI,selpar_slope2_fleet1_D_PH); //vector selpar_L50_fleet1_D_out(1,8);

//vector selpar_slope_fleet1_D_out(1,8);

//vector selpar_L502_fleet1_D_out(1,8);

//vector selpar_slope2_fleet1_D_out(1,8);

init_bounded_number selpar_peak_fleet1_D(selpar_peak_fleet1_D_LO,selpar_peak_fleet1_D_HI,selpar_peak_fleet1_D_PH);

init_bounded_number selpar_top_fleet1_D(selpar_top_fleet1_D_LO,selpar_top_fleet1_D_HI,selpar_top_fleet1_D_PH);

init_bounded_number selpar_ascwid_fleet1_D(selpar_ascwid_fleet1_D_LO,selpar_ascwid_fleet1_D_HI,selpar_ascwid_fleet1_D_PH); init_bounded_number selpar_deswid_fleet1_D(selpar_deswid_fleet1_D_LO,selpar_deswid_fleet1_D_HI,selpar_deswid_fleet1_D_PH); init_bounded_number selpar_init_fleet1_D(selpar_init_fleet1_D_LO,selpar_init_fleet1_D_HI,selpar_init_fleet1_D_PH);

init_bounded_number selpar_final_fleet1_D(selpar_final_fleet1_D_LO,selpar_final_fleet1_D_HI,selpar_final_fleet1_D_PH); vector selpar_peak_fleet1_D_out(1,8);

vector selpar_top_fleet1_D_out $(1,8)$;

vector selpar_ascwid_fleet1_D_out(1,8);

vector selpar_deswid_fleet1_D_out(1,8);

vector selpar_init_fleet1_D_out $(1,8)$;

vector selpar_final_fleet1_D_out(1,8);

//fleet2 landings

matrix sel_fleet2(styr,endyr,1,nages);

init_bounded_number selpar_L50_fleet2(selpar_L50_fleet2_LO,selpar_L50_fleet2_HI,selpar_L50_fleet2_PH);

init_bounded_number selpar_slope_fleet2(selpar_slope_fleet2_LO,selpar_slope_fleet2_HI,selpar_slope_fleet2_PH);

vector selpar_L50_fleet2_out(1,8);

vector selpar_slope_fleet2_out $(1,8)$;

//fleet2 discards

matrix sel_fleet2_D(styr,endyr,1,nages);

//init_bounded_number selpar_L50_fleet2_D(selpar_L50_fleet2_D_LO,selpar_L50_fleet2_D_HI,selpar_L50_fleet2_D_PH);

//init_bounded_number selpar_slope_fleet2_D(selpar_slope_fleet2_D_LO,selpar_slope_fleet2_D_HI,selpar_slope_fleet2_D_PH);

//init_bounded_number selpar_L502_fleet2_D(selpar_L502_fleet2_D_LO, selpar_L502_fleet2_D_HI,selpar_L502_fleet2_D_PH);

//init_bounded_number selpar_slope2_fleet2_D(selpar_slope2_fleet2_D_LO,selpar_slope2_fleet2_D_HI,selpar_slope2_fleet2_D_PH);

//vector selpar_L50_fleet2_D_out(1,8);

$/ /$ vector selpar_slope_fleet2_D_out $(1,8)$;

//vector selpar_L502_fleet2_D_out $(1,8)$;

//vector selpar_slope-_fleet2_D_out(1,8);

init_bounded_number selpar_peak_fleet2_D(selpar_peak_fleet2_D_LO,selpar_peak_fleet2_D_HI,selpar_peak_fleet2_D_PH);

init_bounded_number selpar_top_fleet2_D(selpar_top_fleet2_D_LO,selpar_top_fleet2_D_HI,selpar_top_fleet2_D_PH);

init_bounded_number selpar_ascwid_fleet2_D(selpar_ascwid_fleet2_D_LO,selpar_ascwid_fleet2_D_HI,selpar_ascwid_fleet2_D_PH); init_bounded_number selpar_deswid_fleet2_D(selpar_deswid_fleet2_D_LO,selpar_deswid_fleet2_D_HI,selpar_deswid_fleet2_D_PH); init_bounded_number selpar_init_fleet2_D(selpar_init_fleet2_D_LO,selpar_init_fleet2_D_HI,selpar_init_fleet2_D_PH);

init_bounded_number selpar_final_fleet2_D(selpar_final_fleet2_D_LO,selpar_final_fleet2_D_HI,selpar_final_fleet2_D_PH); vector selpar_peak_fleet2_D_out $(1,8)$;

vector selpar_top_fleet2_D_out $(1,8)$;

vector selpar_ascwid_fleet2_D_out $(1,8)$;

vector selpar_deswid_fleet2_D_out $(1,8)$;

vector selpar_init_fleet2_D_out $(1,8)$;

vector selpar_final_fleet2_D_out $(1,8)$;

//Weighted total selectivity-

//effort-weighted, recent selectivities

vector sel_wgted_L(1,nages); //toward landings

vector sel_wgted_D(1,nages); //toward discards

vector sel_wgted_tot(1,nages);//toward Z, landings plus deads discards

\section{$/ /-$}

//------CPUE Predictions-

vector pred_survey1_cpue(styr_survey1_cpue,endyr_survey1_cpue); matrix N_survey1(styr_survey1_cpue,endyr_survey1_cpue,1, nages); vector pred_fleet1_cpue(styr_fleet1_cpue,endyr_fleet1_cpue); matrix N_fleet1(styr_fleet1_cpue,endyr_fleet1_cpue,1,nages);

//predicted survey1 index (number fish per effort) //used to compute survey1 index $/ /$ predicted fleet1 index (weight fish per effort) //used to compute fleet1 index

//---Catchability (CPUE q's)-

init_bounded_number log_q_survey1(log_q_survey1_LO,log_q_survey1_HI,log_q_survey1_PH);

init_bounded_number log_q_fleet1(log_q_fleet1_LO,log_q_fleet1_HI,log_q_fleet1_PH);

vector log_q_survey1_out $(1,8)$;

vector log_q_fleet1_out(1,8); 


\section{Supplementary Material}

// init_bounded_number q_rate(0.001,0.1,set_q_rate_phase); //KC not estimated so commented out, defleet2ared as number //number q_rate;

//vector q_rate_fcn_rec(styr_rec_cpue,endyr_rec_cpue); //increase due to tefleet1nology creep (saturates in 2003) //KWS

//vector q_rate_fcn_fleet1(styr_fleet1_cpue,endyr_fleet1_cpue); //increase due to tefleet1nology creep (saturates in 2003) //KWS

// init_bounded_number q_DD_beta(0.1,0.9,set_q_DD_phase); //KC not estimated so commented out and defleet2ared as number (below) //number q_DD_beta;

//vector q_DD_fcn(styr,endyr); //density dependent function as a multiple of q (scaled a la Katsukawa and Matsuda. 2003)

//number BO_q_DD; $\quad / / B O$ of ages q_DD_age plus

//vector B_q_DD(styr,endyr); //annual biomass of ages q_DD_age plus

//Fishery dependent random walk catfleet1ability

// init_bounded_vector q_RW_log_dev_rec(styr_rec_cpue,endyr_rec_cpue-1,-3.0,3.0,set_q_RW_phase); //NOT estimated in this model //vector q_RW_log_dev_rec(styr_rec_cpue,endyr_rec_cpue-1);

//vector q_RW_log_dev_fleet1(styr_fleet1_cpue,endyr_fleet1_cpue-1);

//Fishery dependent catfleet1ability over time, may be constant vector q_fleet1(styr_fleet1_cpue,endyr_fleet1_cpue);

\section{$/ /-$}

//---Landings in numbers (total or 1000 fish) and in wgt (klb)-

matrix L_fleet1_num(styr,endyr,1,nages); //landings (numbers) at age

matrix L_fleet1_klb(styr,endyr,1,nages); //landings (1000 lb whole weight) at age

vector pred_fleet1_L_knum(styr,endyr); //yearly landings in 1000 fish summed over ages

vector pred_fleet1_L_klb(styr,endyr); //yearly landings in $1000 \mathrm{lb}$ summed over ages

matrix D_fleet1_num(styr,endyr,1, nages); //discards (numbers) at age

matrix D_fleet1_klb(styr,endyr,1,nages); //discards (1000 lb whole weight) at age

vector pred_fleet1_D_knum(styr,endyr); //yearly discards in 1000 fish summed over ages

vector pred_fleet1_D_klb(styr,endyr); //yearly discards in $1000 \mathrm{lb}$ summed over ages

matrix L_fleet2_num(styr,endyr,1,nages); //landings (numbers) at age

matrix L_fleet2_klb(styr,endyr,1,nages); //landings (1000 lb whole weight) at age

vector pred_fleet2_L_knum(styr,endyr); //yearly landings in 1000 fish summed over ages

vector pred_fleet2_L_klb(styr,endyr); //yearly landings in $1000 \mathrm{lb}$ summed over ages

matrix D_fleet2_num(styr,endyr,1,nages); //discards (numbers) at age

matrix D_fleet2_klb(styr,endyr,1,nages); //discards (1000 lb whole weight) at age

vector pred_fleet2_D_knum(styr,endyr); //yearly discards in 1000 fish summed over ages

vector pred_fleet2_D_klb(styr,endyr); //yearly discards in $1000 \mathrm{lb}$ summed over ages

matrix L_total_num(styr,endyr,1,nages);//total landings in number at age

matrix L_total_klb(styr,endyr,1,nages);//landings in klb at age

vector L_total_knum_yr(styr,endyr); //total landings in 1000 fish by yr summed over ages

vector L_total_klb_yr(styr,endyr); //total landings (klb) by yr summed over ages

matrix D_total_num(styr,endyr,1,nages);//total landings in number at age

matrix D_total_klb(styr,endyr,1,nages);//landings in $\mathrm{klb}$ at age

vector D_total_knum_yr(styr,endyr); //total landings in 1000 fish by yr summed over ages

vector D_total_klb_yr(styr,endyr); //total landings (klb) by yr summed over ages

vector L_proj(1,nages); //total landings at-age for one year projection to get $A B C$

number L_proj_klb; //total landings in klb for one year projection

number L_proj_knum; //total landings in 1000 fish for one year projection

vector D_proj(1,nages); //total landings at-age for one year projection to get $A B C$ number D_proj_klb; //total landings in klb for one year projection

number D_proj_knum; //total landings in 1000 fish for one year projection

////---MSY calcs-

number F_fleet1_prop; //proportion of $F_{-}$sum attributable to fleet1, last $\mathrm{X}$ yr

number F_fleet1_D_prop; //proportion of F_sum attributable to fleet1, last $X \mathrm{yr}$

number F_fleet2_prop; //proportion of F_sum attributable to fleet2, last $\mathrm{X}$ yr 


\section{Supplementary Material}

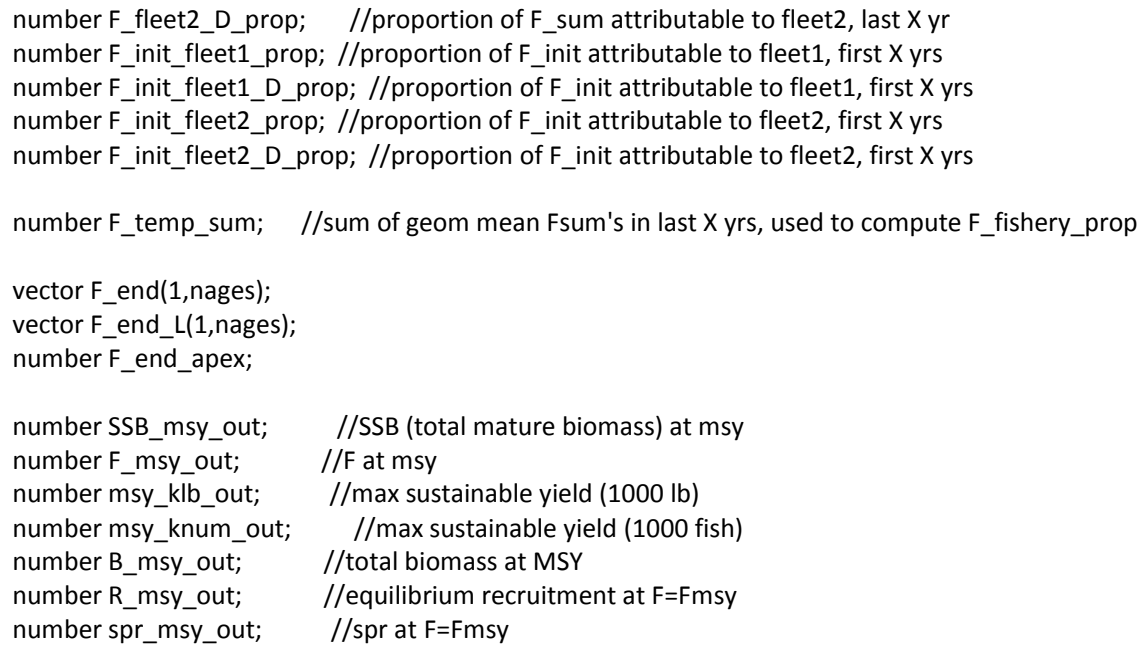




\title{
Supplementary Material
}

\author{
matrix $F($ styr,endyr,1,nages); \\ vector F_proj(1,nages); \\ vector F_D_proj(1,nages); \\ vector Fsum(styr,endyr); \\ vector Fapex(styr,endyr); \\ matrix Z(styr,endyr,1,nages); \\ vector $Z_{-}$proj(1,nages);
}

//Full fishing mortality rate by year

//Max across ages, fishing mortality rate by year (may differ from Fsum bc of dome-shaped sel

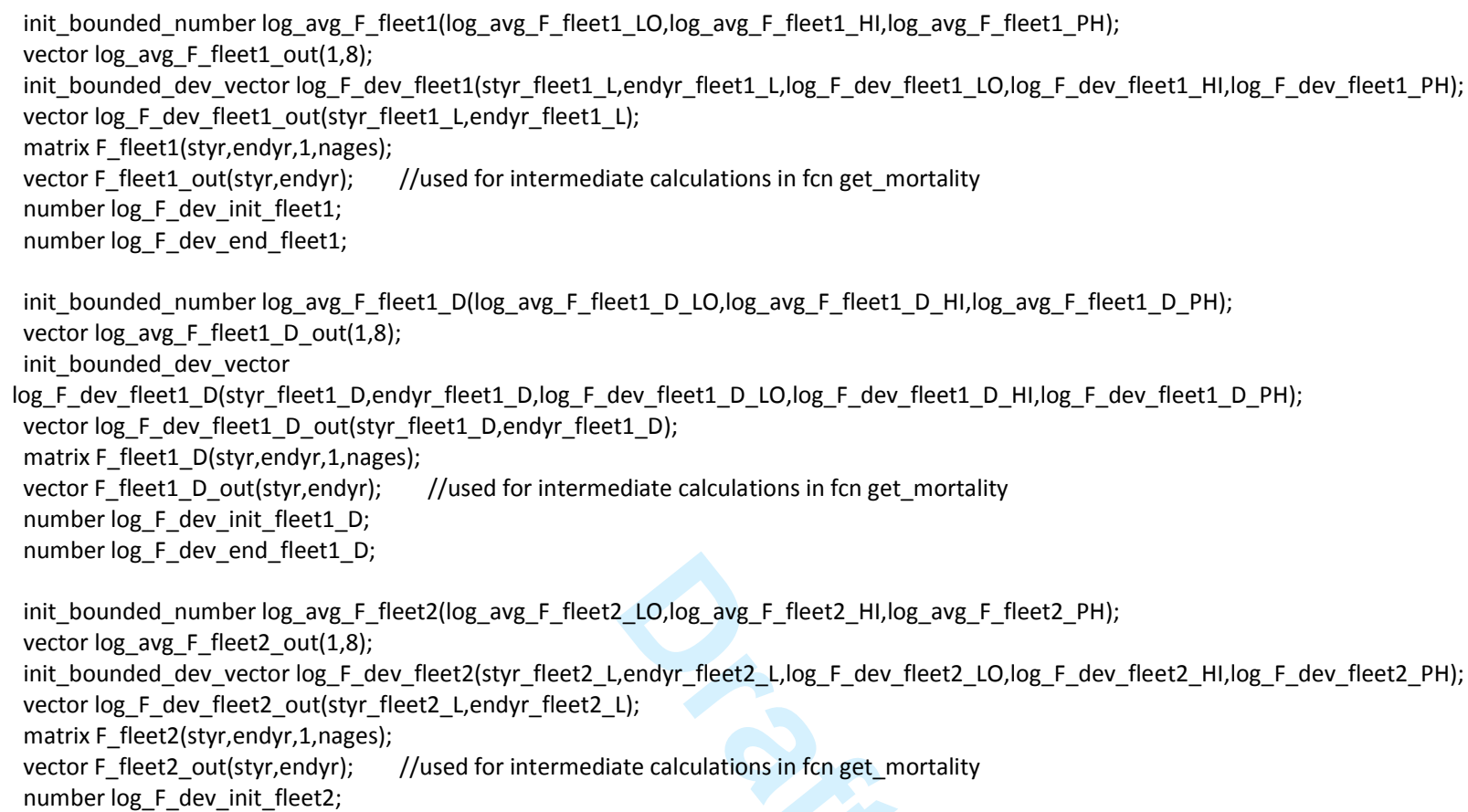




\section{Supplementary Material}

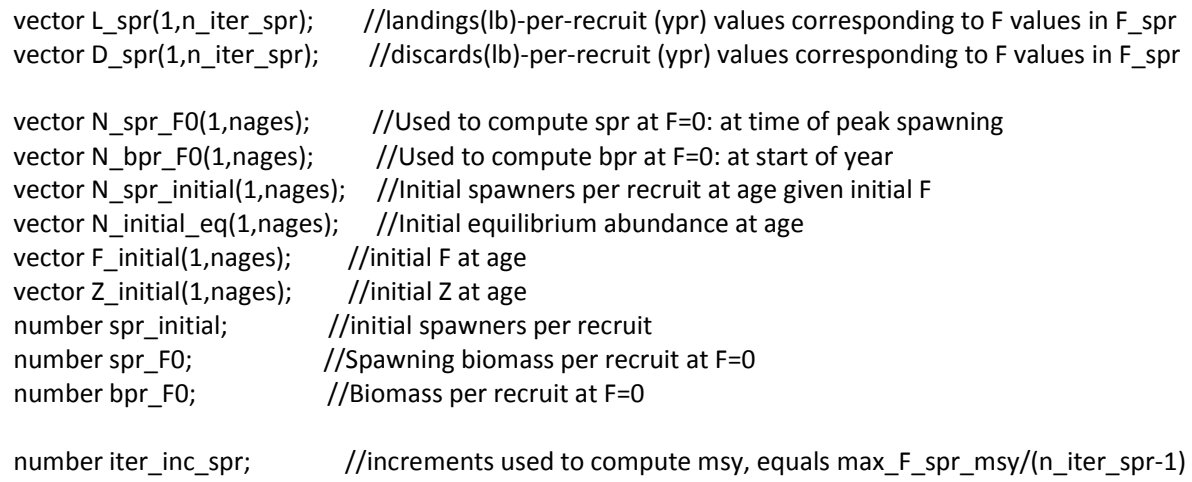




\section{Supplementary Material}

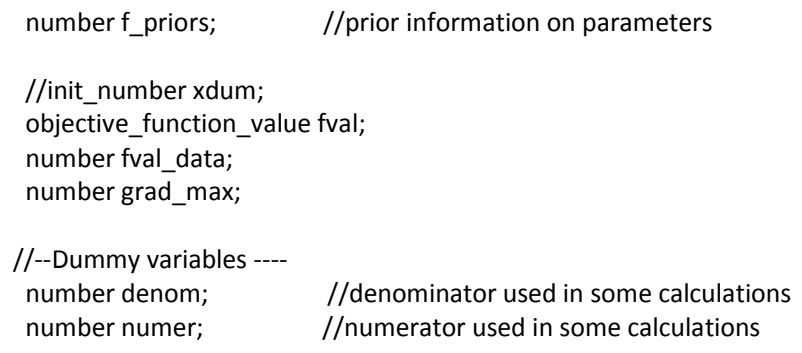

// Set values of fixed parameters or set initial guess of estimated parameters Linf=set_Linf(1);

$\mathrm{K}=$ set_K(1);

t0 $=$ set_to(1);

$M=$ set_M;

$\log \_\mathrm{RO}=$ set_log_RO(1);

steep=set_steep $(1)$;

R_autocorr=set_R_autocorr(1);

rec_sigma=set_rec_sigma(1);

log_q_survey1=set_log_q_survey1(1);

log_q_fleet1=set_log_q_fleet1(1);

//q_rate=set_q_rate;

//q_rate_fon_fleet1=1.0;

//q_DD_beta=set_q_DD_beta;

$/ / q \_D D \_f c n=1.0$;

//q_RW_log_dev_fleet1.initialize();

//if (set_q_rate_phase $<0$ \& q_rate! $=0.0$ )

$/ /\{$

// for (iyear=styr_fleet1_cpue; iyear<=endyr_fleet1_cpue; iyear++)

$/ / \quad\{$ if (iyear $>$ styr_fleet1_cpue \& iyear $<=2003$ )

// $\quad$ //q_rate_fcn_fleet1(iyear) $=\left(1.0+q \_r a t e\right) * q \_r a t e \_f c n \_f l e e t 1($ iyear-1); //compound

$/ /$ q_rate_fcn_fleet1(iyear)=(1.0+(iyear-styr_fleet1_cpue)*q_rate)*q_rate_fcn_fleet1(styr_fleet1_cpue); //linear

// $\quad 3$

// if (iyear>2003) \{q_rate_fcn_fleet1(iyear)=q_rate_fcn_fleet1(iyear-1); 


\section{Supplementary Material}

$/ / \quad\}$

//\} //end q_rate conditional

W_L_fleet1=set_w_L_fleet1;

w_L_fleet2=set_w_L_fleet2;

W_D_fleet1=set_W_D_fleet1;

w_D_fleet2=set_w_D_fleet2;

w_I_survey1=set_w_I_survey1;

w_I_fleet1=set_w_I_fleet1;

w_ac_survey1=set_w_ac_survey1;

w_ac_fleet1 $1=$ set_w_ac_fleet 1 ;

w_ac_fleet2=set_w_ac_fleet2;

w_Nage_init=set_w_Nage_init;

w_rec=set_w_rec;

w_rec_early=set_w_rec_early;

w_rec_end $=$ set_w_rec_end;

w_Ftune=set_w_Ftune;

F_init=set_F_init(1);

log_avg_F_fleet1=set_log_avg_F_fleet1(1);

log_avg_F_fleet2=set_log_avg_F_fleet2(1);

log_avg_F_fleet1_D=set_log_avg_F_fleet1_D(1);

log_avg_F_fleet2_D=set_log_avg_F_fleet2_D(1);

log_F_dev_fleet1=set_log_F_dev_fleet1_vals; log_F_dev_fleet2=set_log_F_dev_fleet2_vals; log_F_dev_fleet1_D=set_log_F_dev_fleet1_D_vals; log_F_dev_fleet2_D=set_log_F_dev_fleet2_D_vals;

selpar_L50_survey1=set_selpar_L50_survey1(1);

selpar_slope_survey1=set_selpar_slope_survey1(1);

selpar_L50_fleet1=set_selpar_L50_fleet1(1);

selpar_slope_fleet1=set_selpar_slope_fleet1(1);

selpar_peak_fleet1_D=set_selpar_peak_fleet1_D(1);

selpar_top_fleet1_D=set_selpar_top_fleet1_D(1);

selpar_ascwid_fleet1_D=set_selpar_ascwid_fleet1_D(1);

selpar_deswid_fleet1_D=set_selpar_deswid_fleet1_D(1);

selpar_init_fleet1_D=set_selpar_init_fleet1_ $D(1)$;

selpar_final_fleet1_D=set_selpar_final_fleet1_D(1);

selpar_L50_fleet2=set_selpar_L50_fleet2(1);

selpar_slope_fleet2=set_selpar_slope_fleet2(1);

selpar_peak_fleet2_D=set_selpar_peak_fleet2_D(1);

selpar_top_fleet2_D=set_selpar_top_fleet2_D(1);

selpar_ascwid_fleet2_D=set_selpar_ascwid_fleet2_D(1);

selpar_deswid_fleet2_D=set_selpar_deswid_fleet2_D(1);

selpar_init_fleet2_D=set_selpar_init_fleet2_D(1);

selpar_final_fleet2_D=set_selpar_final_fleet2_D(1);

sqrt2pi=sqrt(2.*3.14159265)

$\mathrm{g} 2 \mathrm{mt}=0.000001 ; \quad / /$ conversion of grams to metric tons

g2kg=0.001; //conversion of grams to $\mathrm{kg}$

$\mathrm{mt} 2 \mathrm{klb}=2.20462$; //conversion of metric tons to $1000 \mathrm{lb}$

$\mathrm{mt} 2 \mathrm{lb}=\mathrm{mt} 2 \mathrm{klb} * 1000.0 ; \quad / /$ conversion of metric tons to $\mathrm{lb}$

$\mathrm{g} 2 \mathrm{klb}=\mathrm{g} 2 \mathrm{mt}^{*} \mathrm{mt} 2 \mathrm{klb} ; \quad / /$ conversion of grams to $1000 \mathrm{lb}$

dzero $=0.00001$;

huge_number $=1.0 \mathrm{e}+10$; 


\section{Supplementary Material}

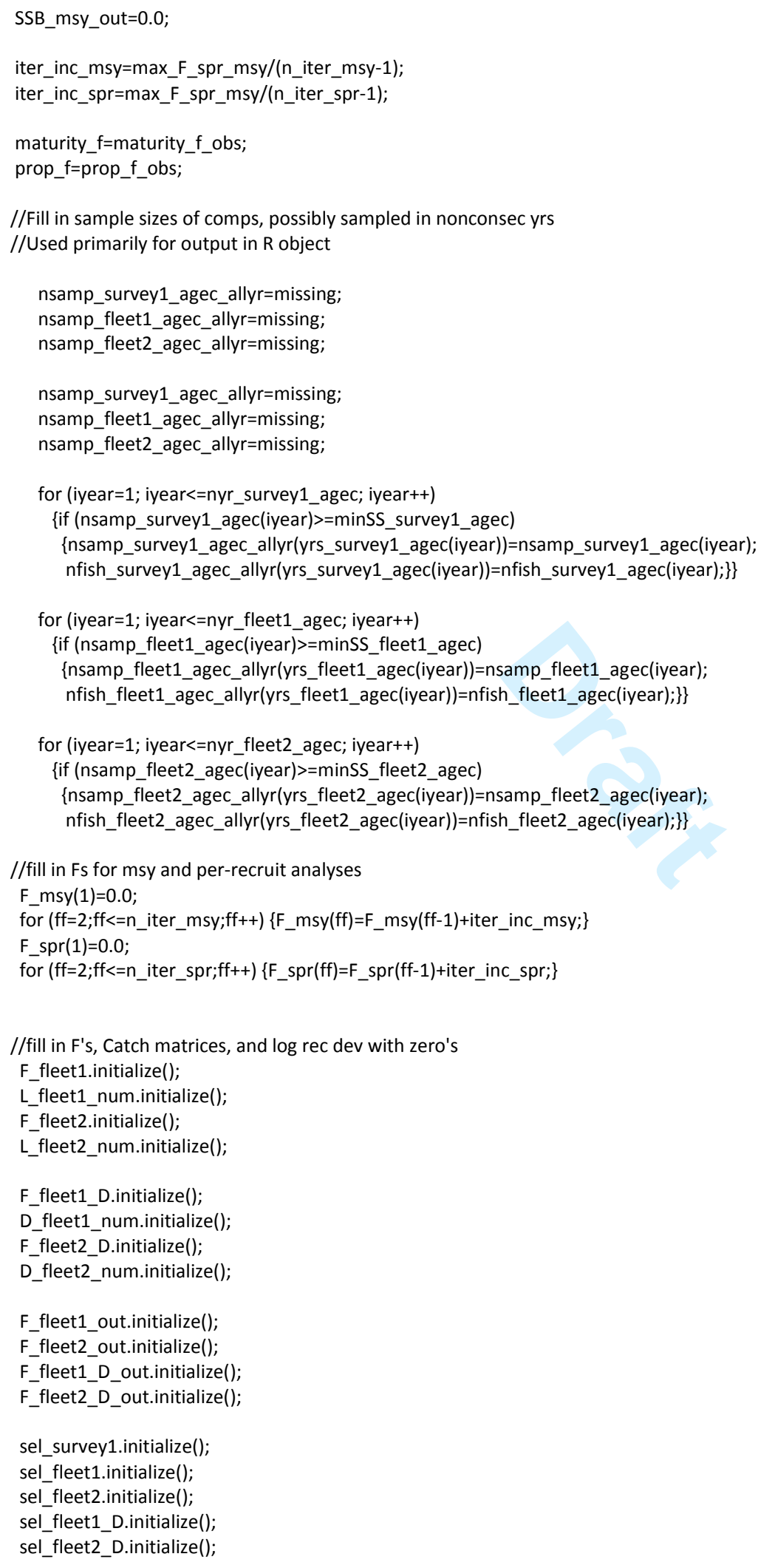




\section{Supplementary Material}

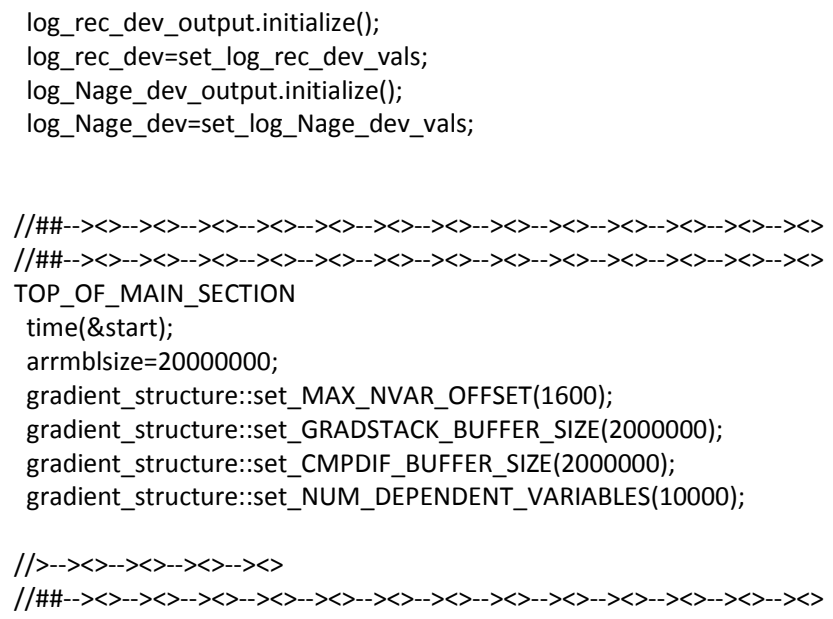




\section{Supplementary Material}

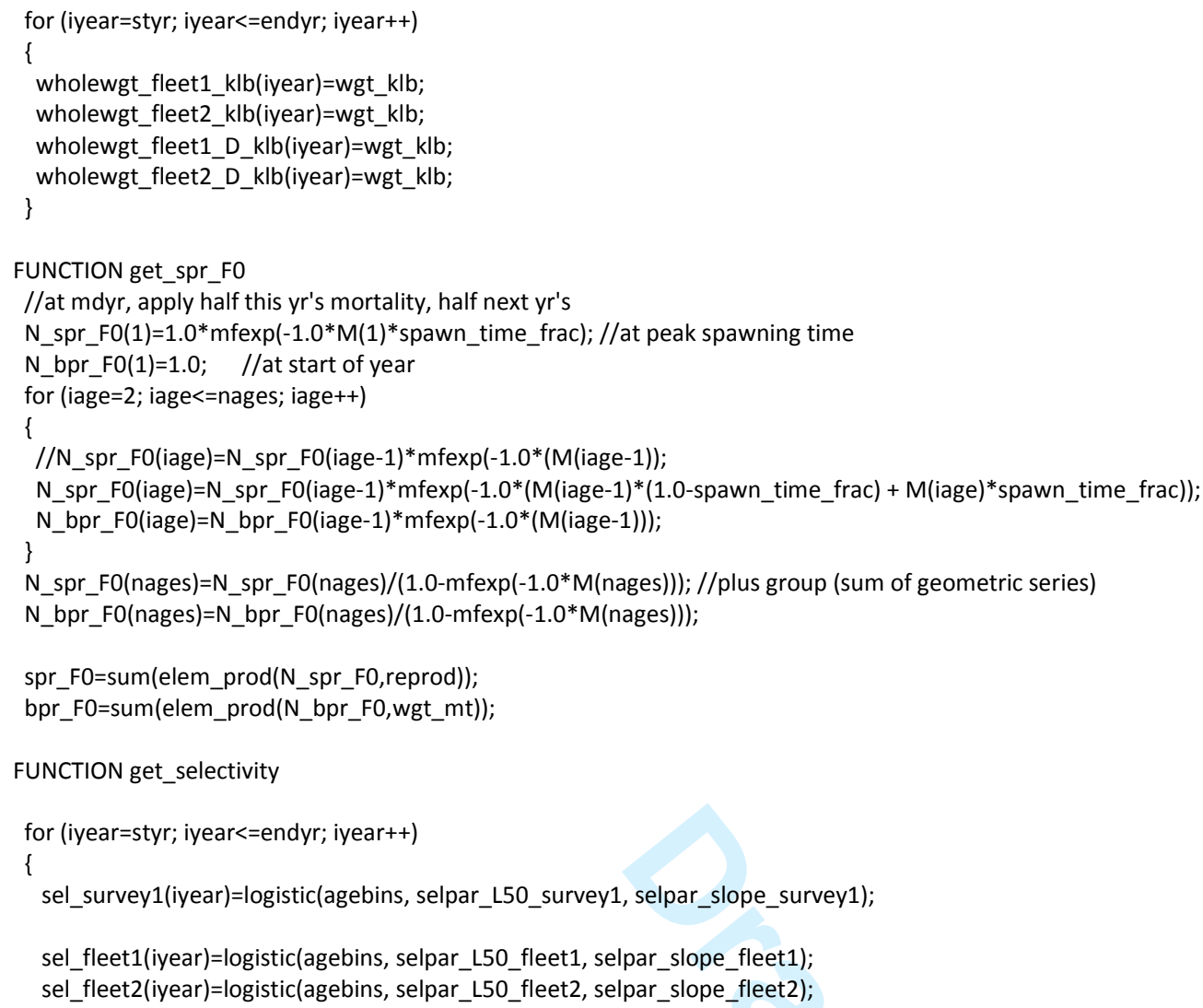




\section{Supplementary Material}

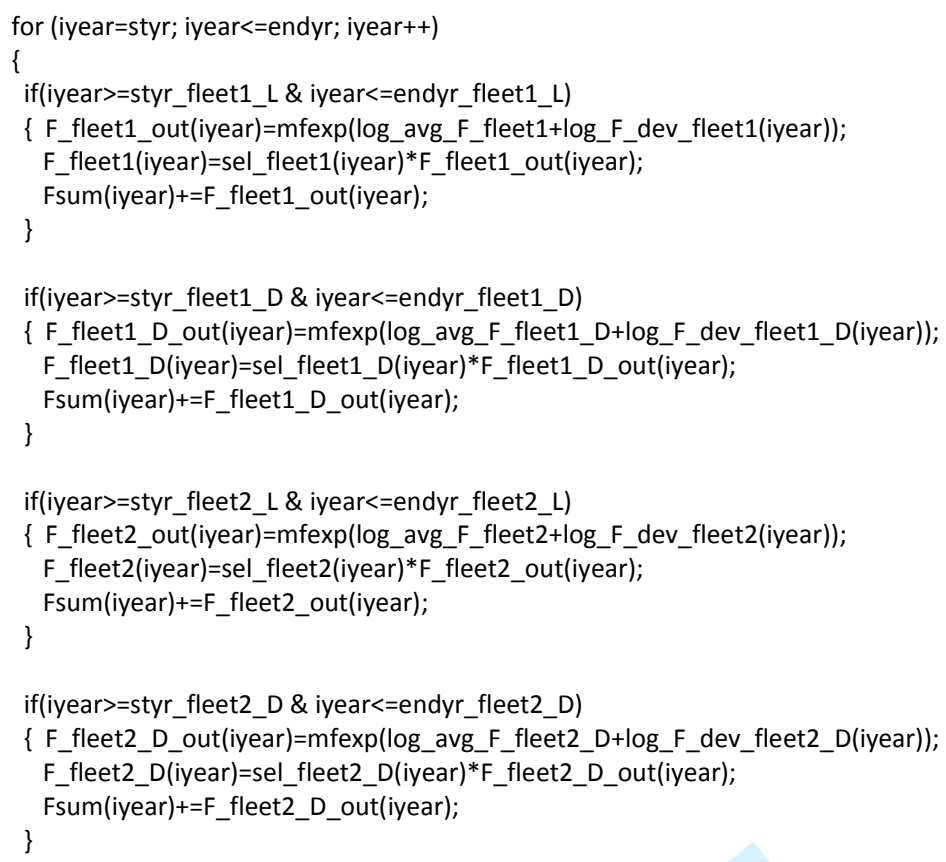




\section{Supplementary Material}

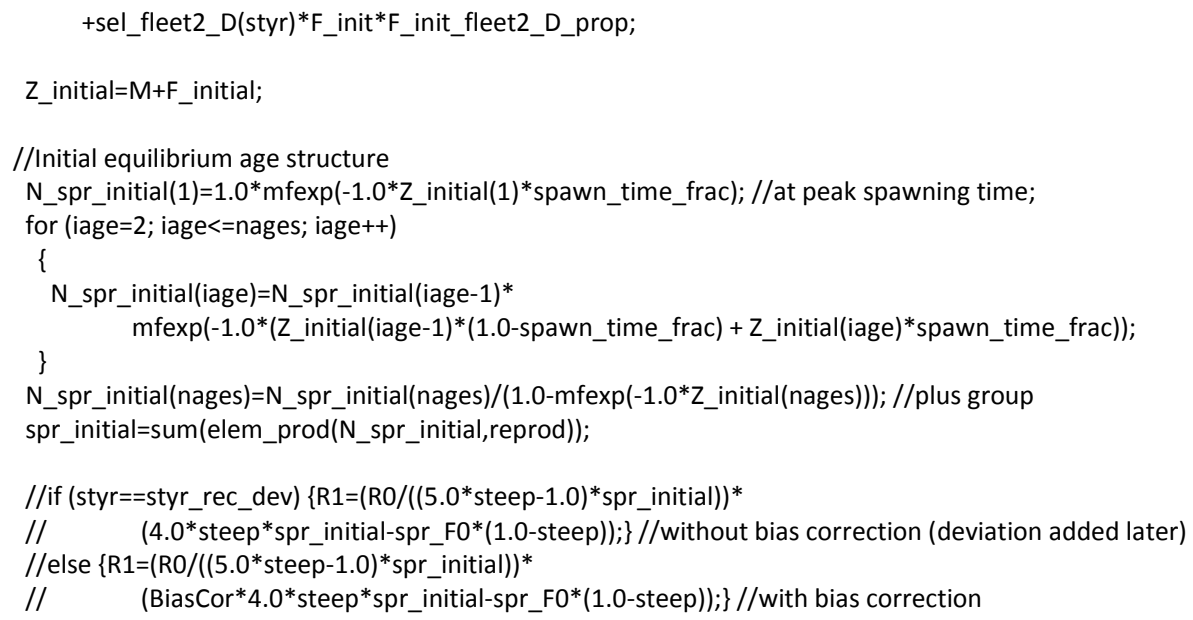




\section{Supplementary Material}

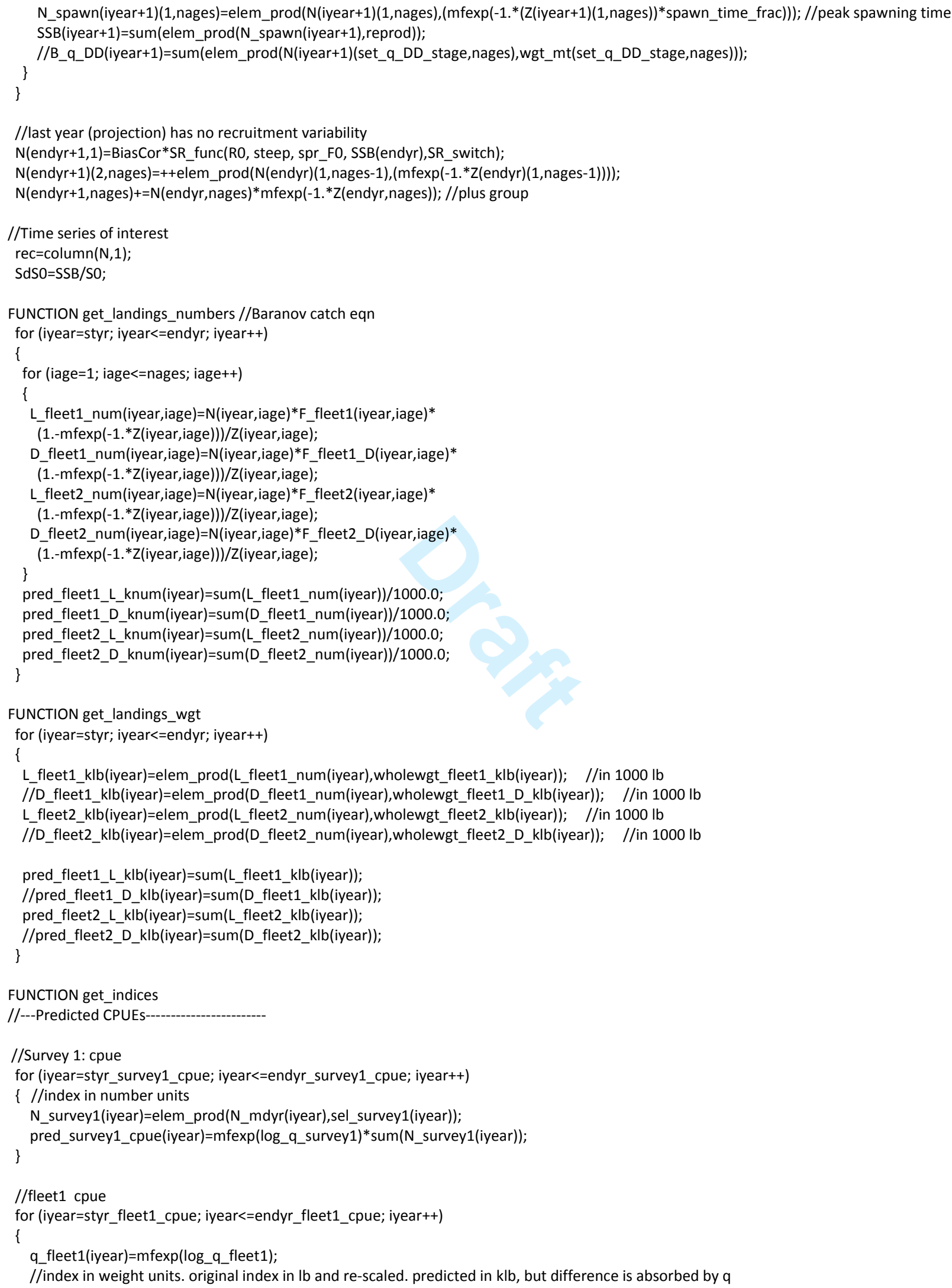




\title{
Supplementary Material
}

\author{
N_fleet1(iyear)=elem_prod(elem_prod(N_mdyr(iyear),sel_fleet1(iyear)), wholewgt_fleet1_klb(iyear)); \\ //N_fleet1(iyear)=elem_prod(N_mdyr(iyear),sel_fleet1(iyear));

log_F_dev_end_fleet1=sum(log_F_dev_fleet1((endyr-selpar_n_yrs_wgted+1),endyr))/selpar_n_yrs_wgted; log_F_dev_end_fleet1_D=sum(log_F_dev_fleet1_D((endyr-selpar_n_yrs_wgted +1$)$,endyr))/selpar_n_yrs_wgted; log_F_dev_end_fleet2=sum (log_F_dev_fleet2 ((endyr-selpar_n_yrs_wgted +1$)$,endyr))/selpar_n_yrs_wgted; log_F_dev_end_fleet2_D=sum(log_F_dev_fleet2_D((endyr-selpar_n_yrs_wgted+1),endyr))/selpar_n_yrs_wgted; 


\section{Supplementary Material}

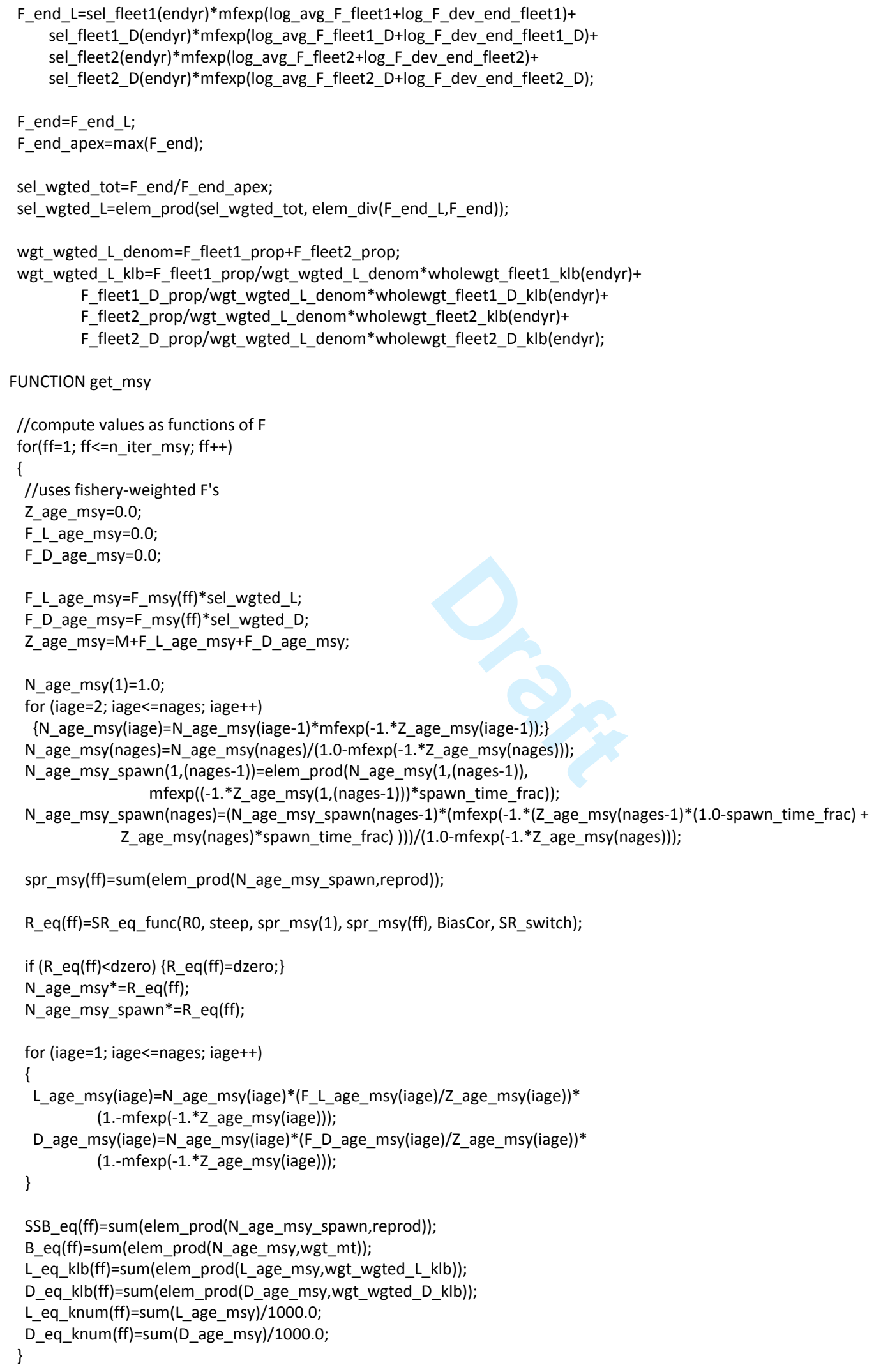




\section{Supplementary Material}

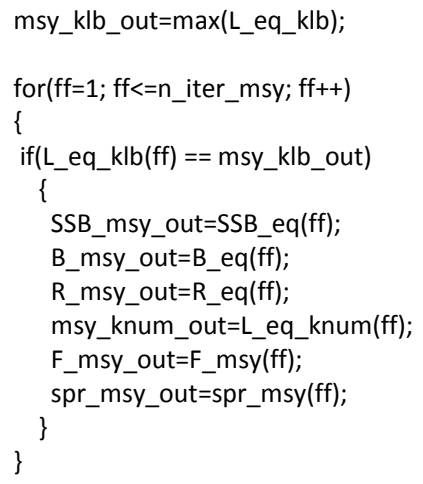

L_total_num=L_fleet1_num+L_fleet2_num; //landings at age in number fish L_total_klb=L_fleet1_klb+L_fleet1_klb; //landings at age in klb whole weight D_total_num=D_fleet1_num+D_fleet2_num; //discards at age in number fish //D_total_klb=D_fleet1_klb+D_fleet1_klb; //discards at age in klb whole weight

$B(e n d y r+1)=e l e m \_p r o d(N(e n d y r+1)$, wgt_mt); $\operatorname{tot} \mathrm{N}($ endyr+1) $=\operatorname{sum}(\mathrm{N}($ endyr+1)); totB $($ endyr+1)=sum $(B($ endyr+1));

// steep_sd=steep;

// fullF_sd=Fsum;

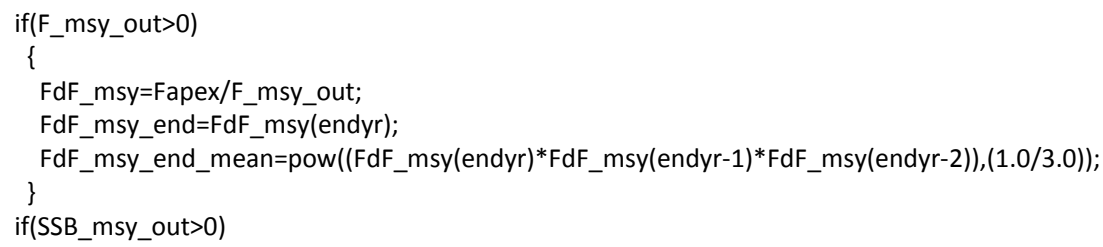




\section{Supplementary Material}

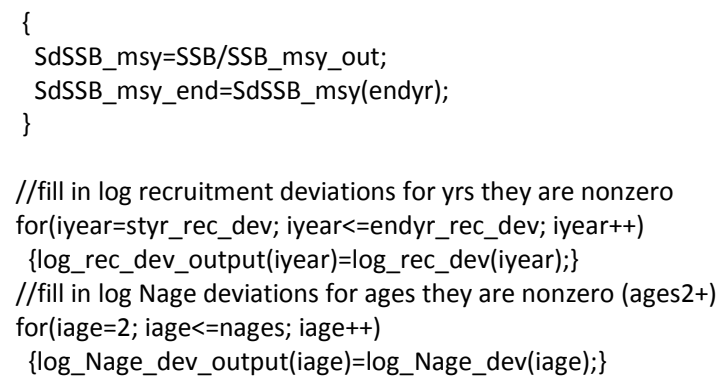




\section{Supplementary Material}

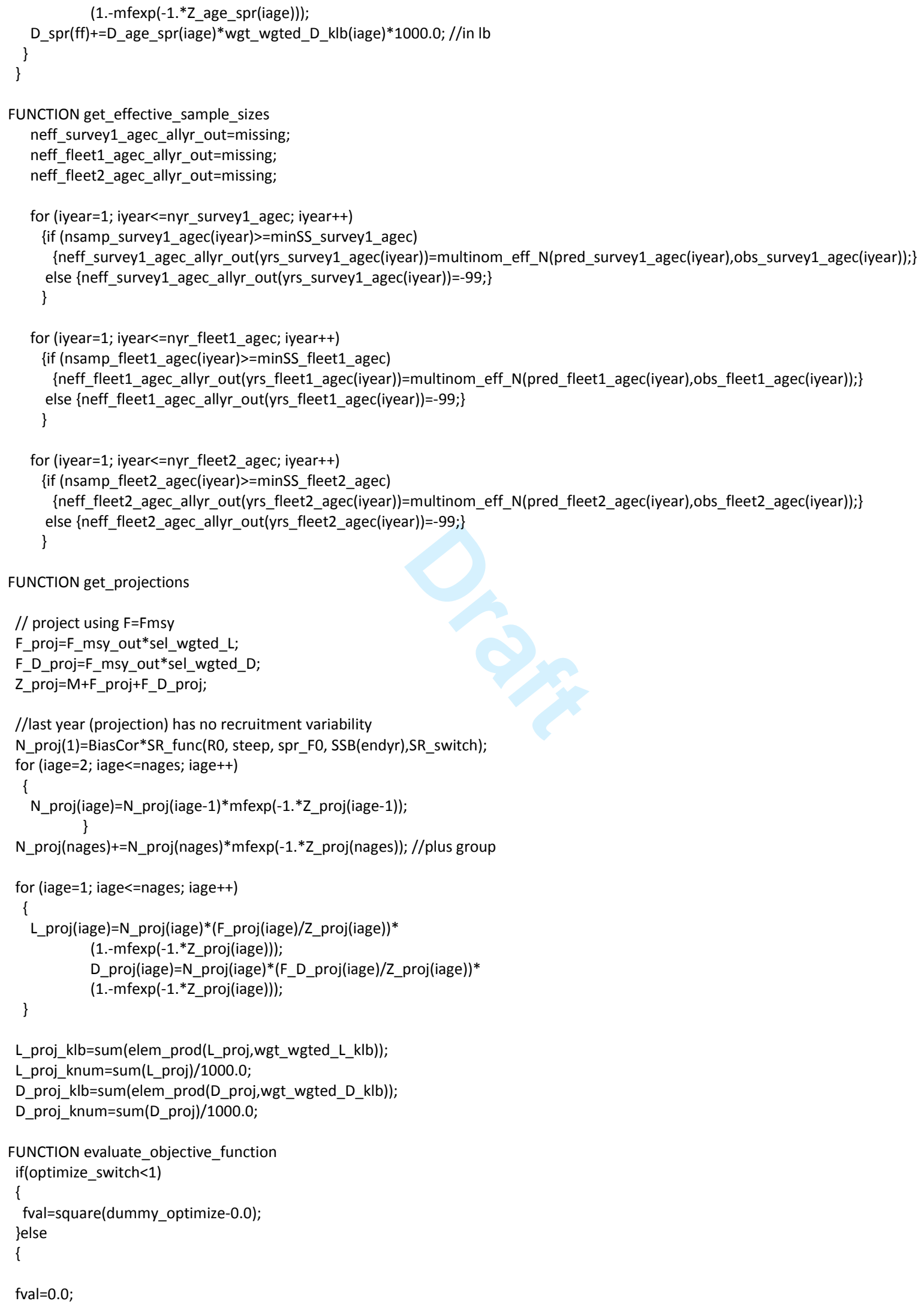




\section{Supplementary Material}

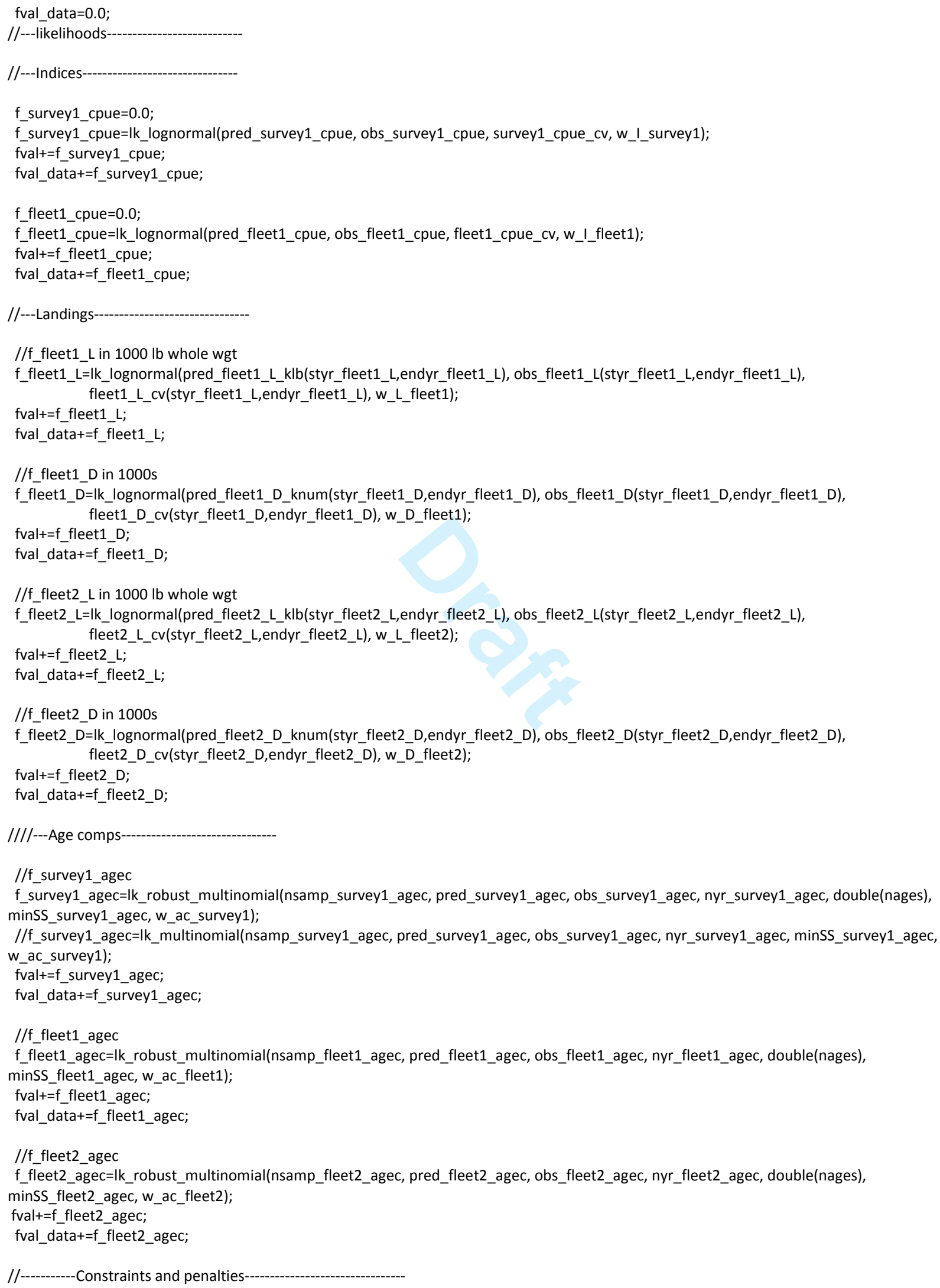




\section{Supplementary Material}

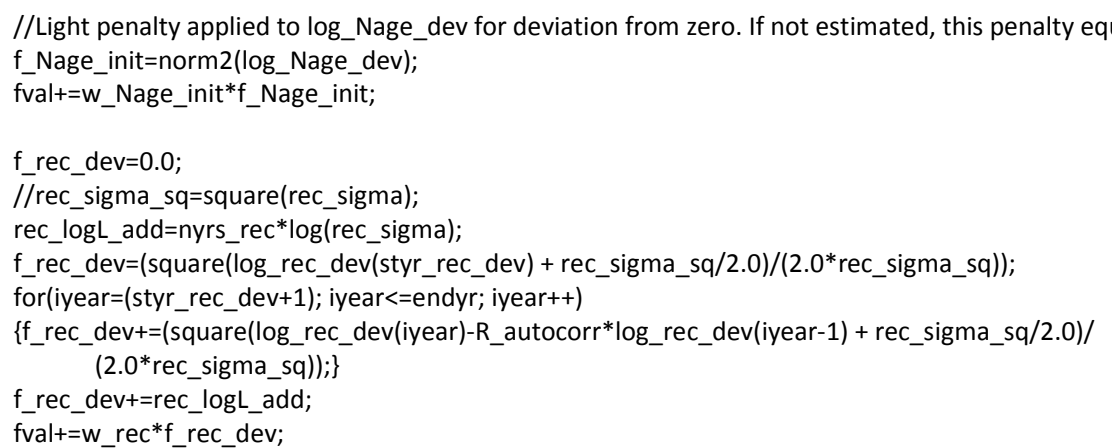

f_rec_dev_early $=0.0 ; / /$ possible extra constraint on early rec deviations 


\section{Supplementary Material}

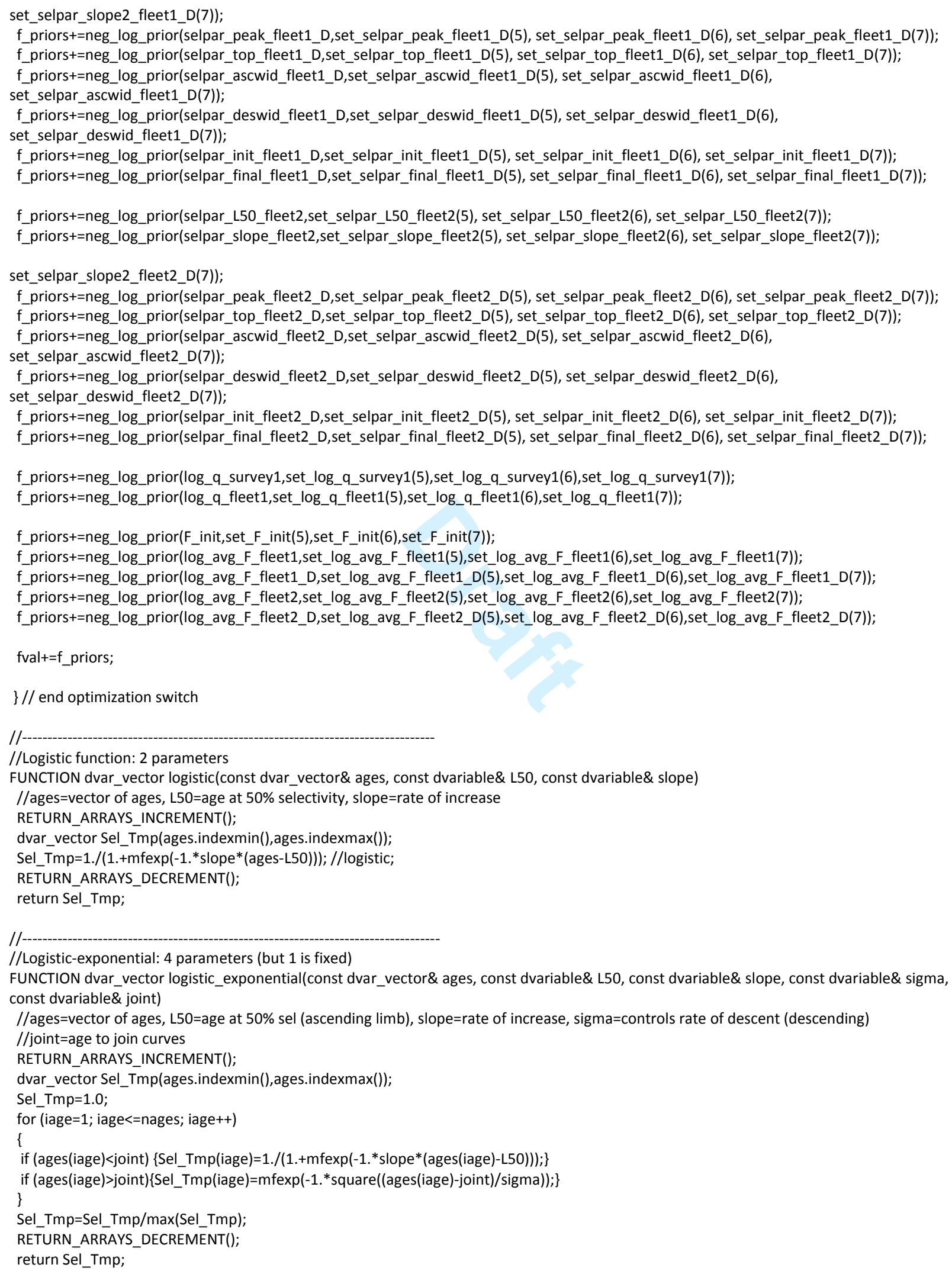




\section{Supplementary Material}

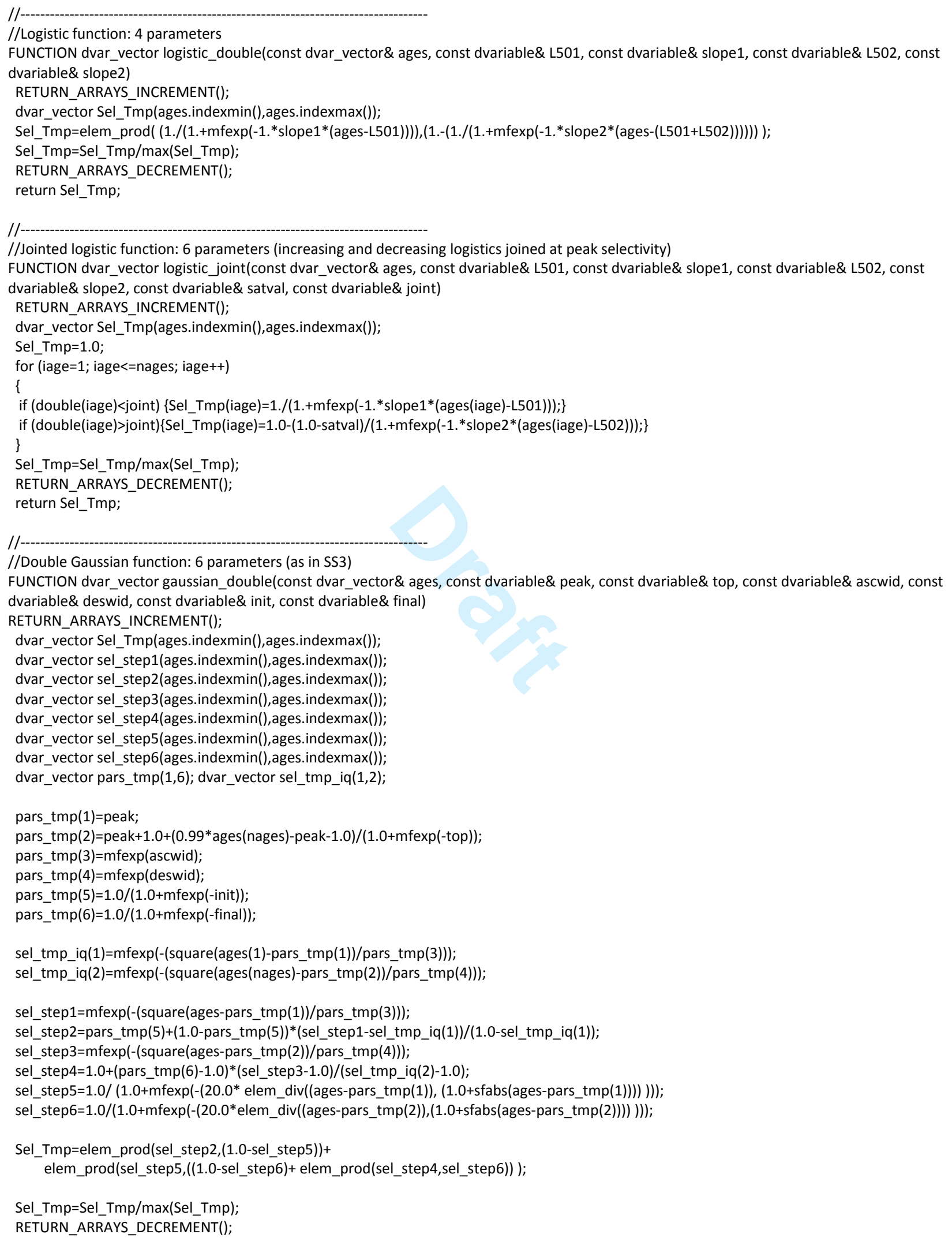




\section{Supplementary Material}

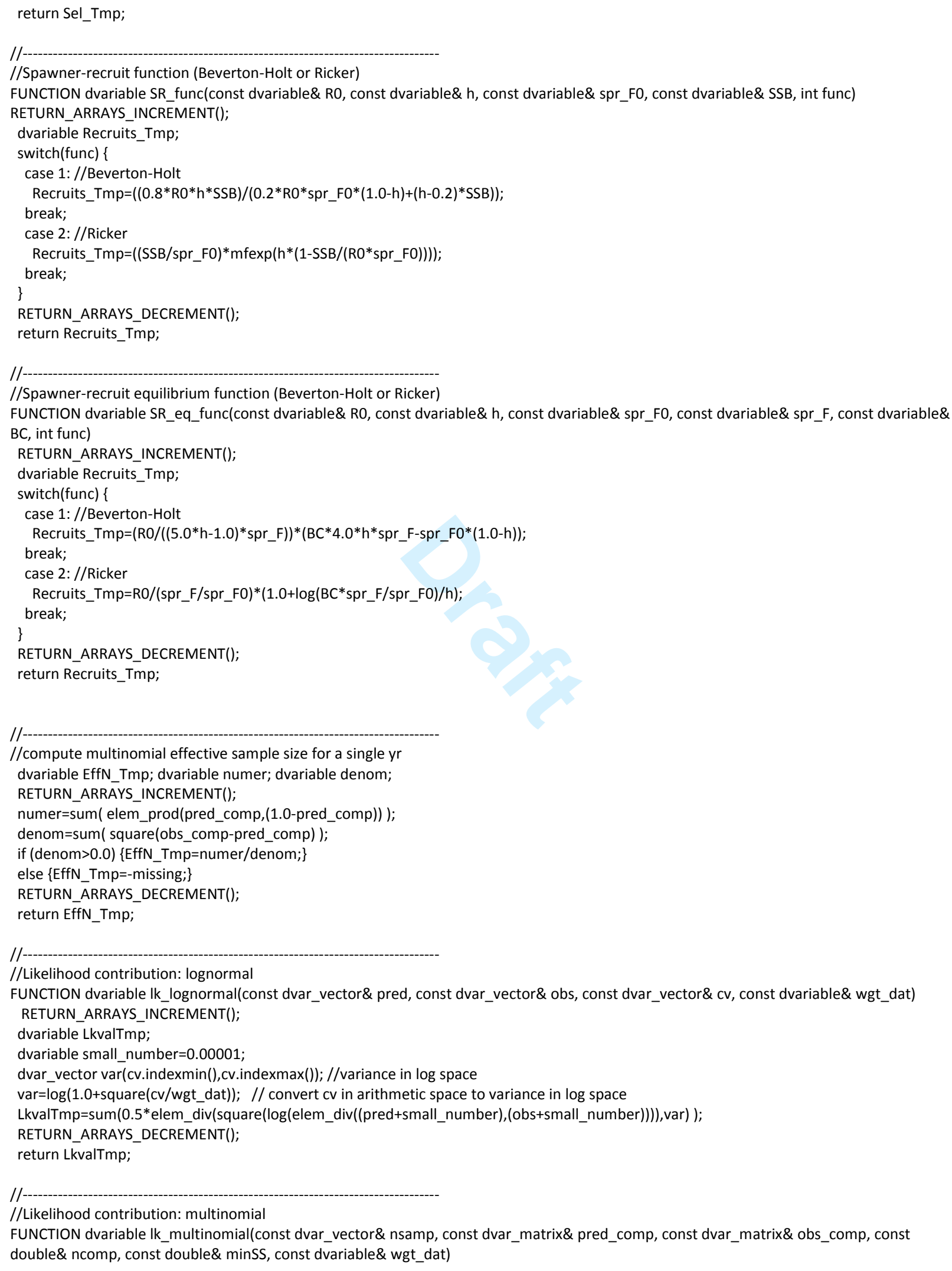




\section{Supplementary Material}

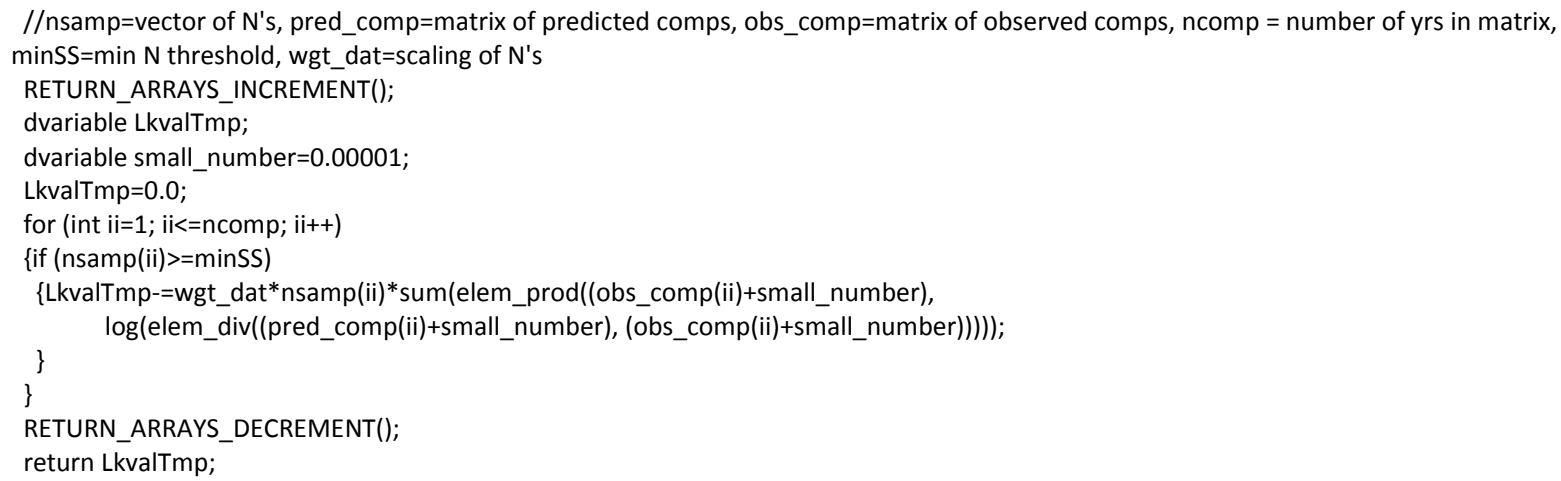




\section{Supplementary Material}

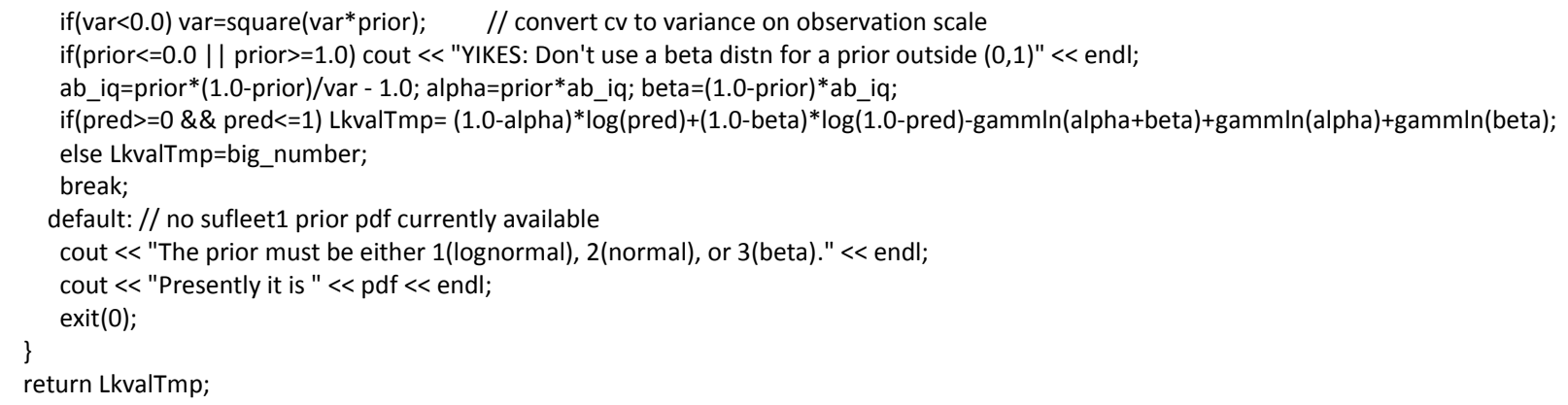




\section{Supplementary Material}

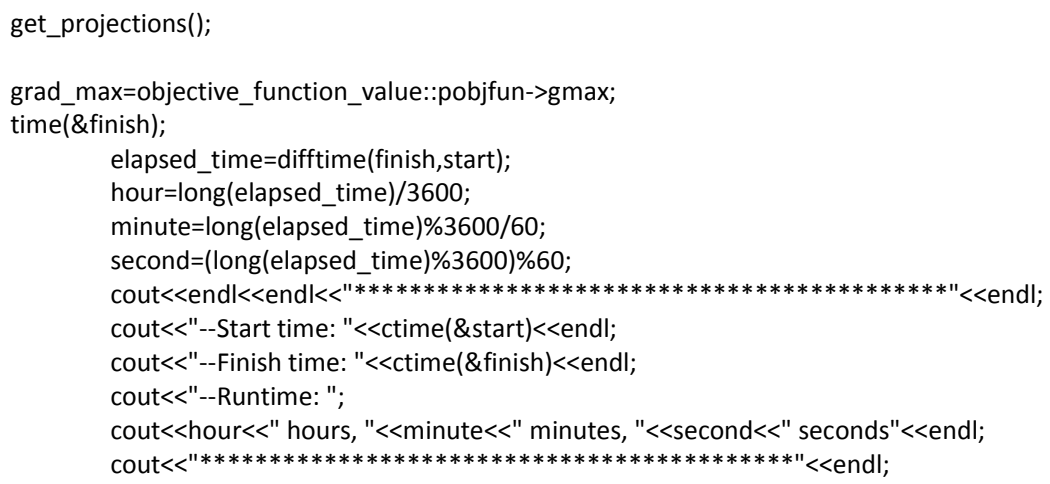




\section{Supplementary Material}

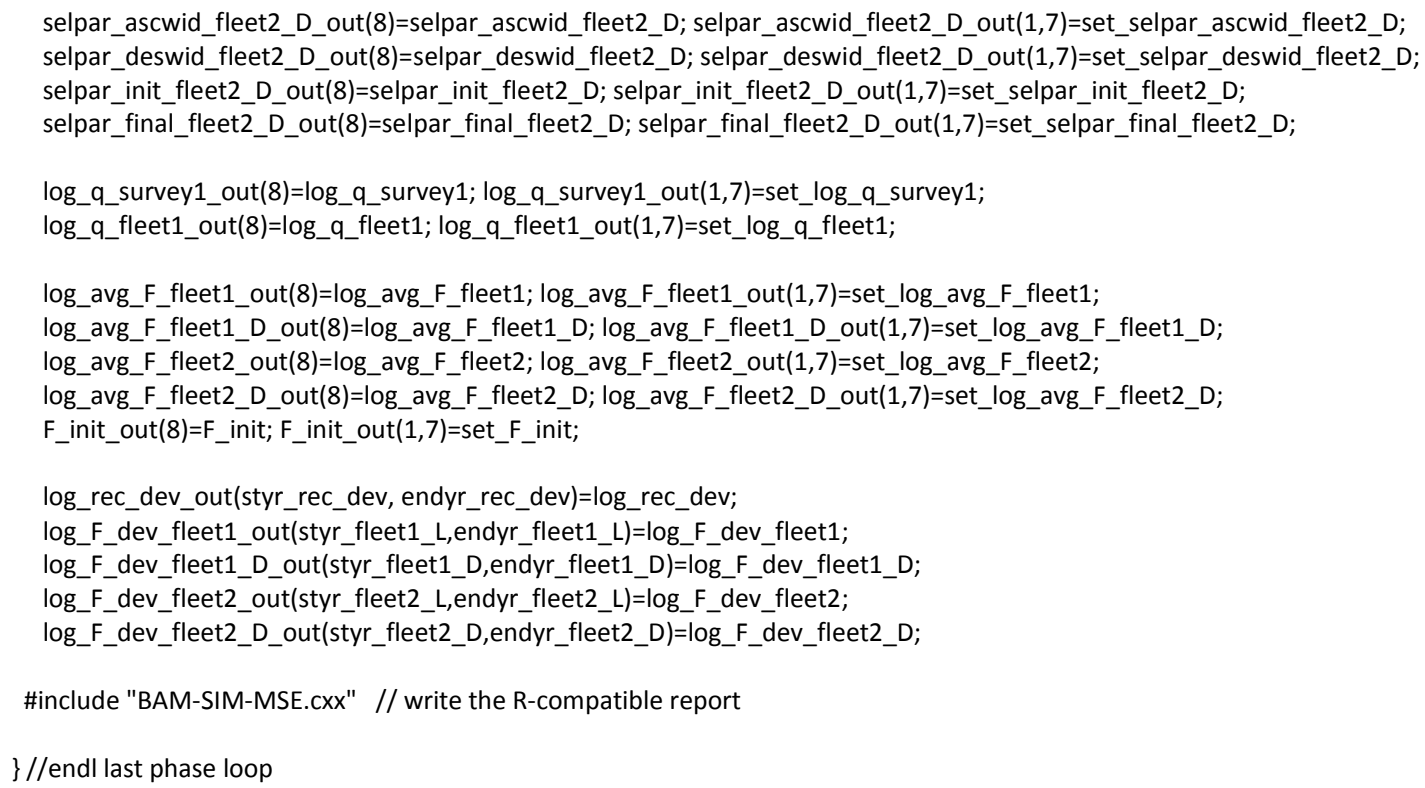

